\title{
PURE NASH EQUILIBRIA IN CONCURRENT DETERMINISTIC GAMES*
}

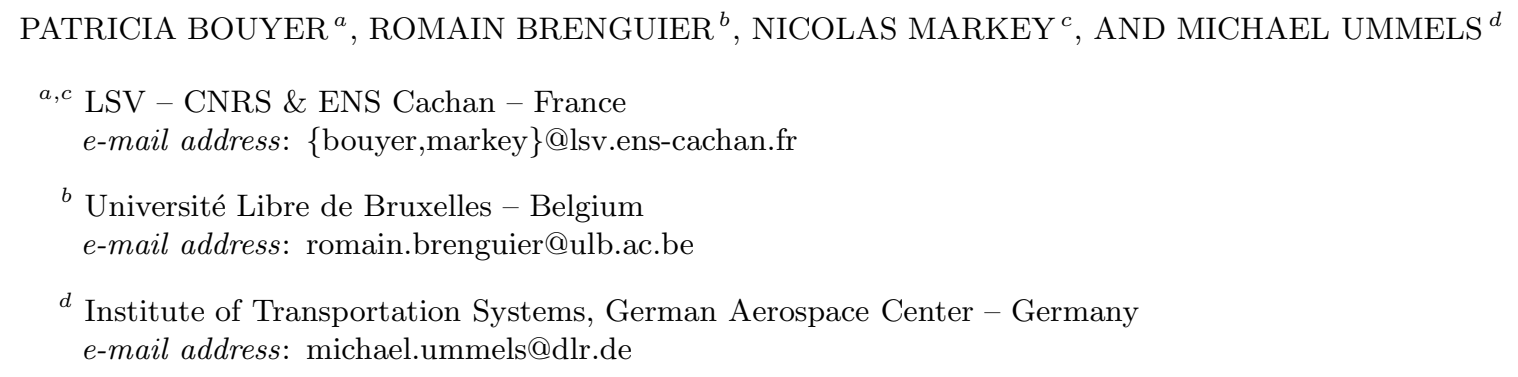

\begin{abstract}
We study pure-strategy Nash equilibria in multi-player concurrent deterministic games, for a variety of preference relations. We provide a novel construction, called the suspect game, which transforms a multi-player concurrent game into a two-player turnbased game which turns Nash equilibria into winning strategies (for some objective that depends on the preference relations of the players in the original game). We use that transformation to design algorithms for computing Nash equilibria in finite games, which in most cases have optimal worst-case complexity, for large classes of preference relations. This includes the purely qualitative framework, where each player has a single $\omega$-regular objective that she wants to satisfy, but also the larger class of semi-quantitative objectives, where each player has several $\omega$-regular objectives equipped with a preorder (for instance, a player may want to satisfy all her objectives, or to maximise the number of objectives that she achieves.)
\end{abstract}

\section{INTRODUCTION}

Games (and especially games played on graphs) have been intensively used in computer science as a powerful way of modelling interactions between several computerised systems [39, 24]. Until recently, more focus had been put on the study of purely antagonistic games (a.k.a. zero-sum games), which conveniently represent systems evolving in a (hostile)

2012 ACM CCS: [Theory of computation]: Theory and algorithms for application domainsAlgorithmic game theory and mechanism design - Algorithmic game theory / Exact and approximate computation of equilibria; Logic; [Software and its engineering]: Software organization and propertiesSoftware functional properties-Formal methods-Model checking.

Key words and phrases: Nash equilibria, concurrent games, qualitative objectives, ordered objectives.

* This article is an extended version of several works that appeared as [4, 5, 6]. Most of the work reported here was done while the second and fourth authors were students at LSV..

This work has been partly supported by ESF LogiCCC project GASICS, ERC Starting Grant EQualIS (308087), ERC Starting Grant inVEST (279499), and EU FP7 project Cassting (601148). 
environment. In this zero-sum games setting, the objectives of both players are opposite: the aim of one player is to prevent the other player from achieving her own objective.

Over the last ten years, games with non-zero-sum objectives have come into the picture: they allow for conveniently modelling complex infrastructures where each individual system tries to fulfil its own objectives, while still being subject to uncontrollable actions of the surrounding systems. As an example, consider a wireless network in which several devices try to send data: each device can modulate its transmit power, in order to maximise its bandwidth and reduce energy consumption as much as possible. In that setting, focusing only on optimal strategies for one single agent may be too narrow. Game-theoreticians have defined and studied many other solution concepts for such settings, of which Nash equilibrium [35] is the most prominent. A Nash equilibrium is a strategy profile where no player can improve the outcome of the game by unilaterally changing her strategy. In other terms, in a Nash equilibrium, each individual player has a satisfactory strategy. Notice that Nash equilibria need not exist or be unique, and are not necessarily optimal: Nash equilibria where all players lose may coexist with more interesting Nash equilibria. Finding constrained Nash equilibria (e.g., equilibria in which some players are required to win) is thus an interesting problem for our setting.

In this paper, we report on our recent contributions on the computation of Nash equilibria in concurrent games (preliminary works appeared as [4, 5, 6]). Concurrent games played on graphs are a general model for interactive systems, where the agents take their decision simultaneously. Therefore concurrent games subsume turn-based games, where in each state, only one player has the decision for the next move. One motivation for concurrent games is the study of timed games (which are games played on timed automata [3, 1]): the semantics of a timed game is naturally given as a concurrent game (the players all choose simultaneously a delay and an action to play, and the player with the shortest delay decides for the next move - this mechanism cannot be made turn-based since we cannot fix a priori the player who will choose the smallest delay); the region-based game abstraction which preserves Nash equilibria also requires the formalism of concurrent games [5, 7]. Multi-agent infrastructures can be viewed as distributed systems, which can naturally be modelled as concurrent games.

Our contributions. The paper focuses on concurrent deterministic games and on pure Nash equilibria, that is, strategy profiles which are deterministic (as opposed to randomised). In this work we assume strategies only depend on the set of states which is visited, and not on the actions that have been played. This is a partial-information hypothesis which we believe is relevant in the context of distributed systems, where only the effect of the actions can be seen by the players. We will discuss in more detail all these choices in the conclusion.

In the context exposed above, we develop a complete methodology for computing pure Nash equilibria in (finite) games. First, in Section 4, we propose a novel transformation of the multi-player concurrent game (with a preference relation for each player) into a twoplayer zero-sum turn-based game, which we call the suspect game. Intuitively, in the suspect game, one of the players suggests a global move (one action per player of the original game), with the aim to progressively build a Nash equilibrium; while the second player aims at proving that what the first player proposes is not a Nash equilibrium. This transformation can be applied to arbitrary concurrent games (even those with infinitely many states) and preference relations for the players, and it has the property that there is a correspondence between Nash equilibria in the original game and winning strategies in the transformed 


\begin{tabular}{|c|c|c|}
\hline Objective & Value & (Constrained) Existence of Nash Eq. \\
\hline Reachability & P-c. 32 & NP-c. (Sect. [5.1) \\
\hline Safety & P-c. 32 & NP-c. (Sect. 5.2) \\
\hline Büchi & P-c. 32 & P-c. (Sect. 5.3) \\
\hline co-Büchi & P-c. 32 & NP-c. (Sect. 5.4) \\
\hline Parity & $U P \cap$ co-UP 28$]$ & $\mathrm{P}_{\|}^{\mathrm{NP}}$-c. ${ }_{-1}^{1}($ Sect. 5.6) \\
\hline Streett & co-NP-c. [18] & $P_{\|}^{N P}-h$. and in PSPACE \\
\hline Rabin & NP-c. 18 & $\mathrm{P}_{\|}^{\mathrm{NP}}$-c. (Sect. [5.6) \\
\hline Muller & PSPACE-c. [27] & PSPACE-c. \\
\hline Circuit & PSPACE-c. [27] & PSPACE-c. (Sect. 5.5]) \\
\hline Det. Büchi Automata & P-c. & PSPACE-h. (Sect. 5.7) and in EXPTIME \\
\hline Det. Rabin Automata & NP-c. & PSPACE-h. and in EXPTIME (Sect. 5.7) \\
\hline
\end{tabular}

TABLE 1. Summary of the complexities for single objectives

\begin{tabular}{r|c|c|c}
\hline Preorder & Value & Existence of NE & Constr. Exist. of NE \\
\hline Maximise, Disj. & P-c. (Sect6.2) & P-c. (Sect6.2) & P-c. (Sect6.2) \\
Subset & P-c. (Sect. 6.3) & P-c. (Sect6.2) & P-c. (Sect 6.2) \\
Conj., Lexicogr. & P-c. (Sect. 6.3) & P-h., in NP (Sect. 6.4) & NP-c. (Sect. 6.4) \\
Counting & coNP-c. (Sect. 6.4) & NP-c. (Sect. 6.4) & NP-c. (Sect. 6.4) \\
Mon. Bool. Circuit & coNP-c. (Sect. 6.4) & NP-c. (Sect. 6.4) & NP-c. (Sect. 6.4) \\
Boolean Circuit & PSPACE-c. (Sect. 6.1) & PSPACE-c. (Sect. 6.1) & PSPACE-c. (Sect. 6.1) \\
\hline
\end{tabular}

TABLE 2. Summary of the results for ordered Büchi objectives

\begin{tabular}{r|c|c}
\hline Preorder & Value & (Constrained) Exist. of NE \\
\hline Disjunction, Maximise & P-c. (Sect. 7.2) & NP-c. (Sect. 7.2) \\
Subset & PSPACE-c. (Sect. 7.1) & NP-c. (Sect. 7.2) \\
$\begin{array}{r}\text { Conjunction, Counting, Lexicogr. } \\
\text { (Monotonic) Boolean Circuit }\end{array}$ & PSPACE-c. (Sect. 7.1) & PSPACE-c. (Sect. 7.1) \\
\hline
\end{tabular}

TABLE 3. Summary of the results for ordered reachability objectives

two-player turn-based game. The winning condition in the suspect game of course depends on the preference relations of the various players in the original game.

Then, using that construction we develop (worst-case) optimal-complexity algorithms for deciding the existence of (constrained) Nash equilibria in finite games for various classes of preference relations. In Section [5, we focus on qualitative $\omega$-regular objectives, i.e., preference relations are given by single objectives (which can be reachability, Büchi, parity, etc), and it is better for a player to satisfy her objective than to not satisfy her objective. We prove the whole set of results which are summarised in the second column of Table 1 (the first column summarises the complexity in the zero-sum two-player setting - called the value problem). Among the results obtained this way, the constrained Nash equilibrium existence problem is NP-complete in finite games with single reachability or safety objectives, while it is PTIME-complete for single Büchi objectives. 


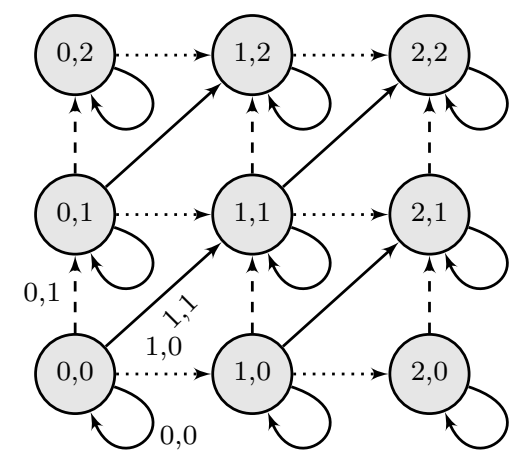

FiguRE 1. A simple game-model for the wireless network

In Sections 6 and 7, we extend the previous qualitative setting to the semi-quantitative setting of ordered objectives. An ordered objective is a set of Büchi (or reachability) objectives and a preorder on this set. The preference relation given by such an ordered objective is then given by the value of the plays (w.r.t. the objectives) in that preorder. Preorders of interest are for instance conjunction, disjunction, lexicographic order, counting preorder, maximise preorder, subset preorder, or more generally preorders given as Boolean circuits. We provide algorithms for deciding the existence of Nash equilibria for ordered objectives, with (in most cases) optimal worst-case complexity. These algorithms make use of the suspect-game construction. The results are listed in Table 2 for Büchi objectives and in Table 3 for reachability objectives.

Examples. Back to the earlier wireless network example, we can model a simple discretised version of it as follows. From a state, each device can increase (action 1) or keep unchanged (action 0) its power: the arena of the game is represented for two devices and two levels of power on Figure 1 (labels of states are power levels). This yields a new bandwidth allocation (which depends on the degradation due to the other devices) and a new energy consumption. The satisfaction of each device is measured as a compromise between energy consumption and bandwidth allocated, and it is given by a quantitative payoff function.2 This can be transformed into Büchi conditions and a preorder on them. There are basically two families of pure Nash equilibria in this system: the one where the two players choose to go and stay forever in state $(1,1)$; and the one where the two players go to state $(2,2)$ and stay there forever.

We describe another example, the medium access control, that involves qualitative objectives. It was first given a game-theoretic model in [31. Several users share the access

\footnotetext{
${ }^{1}$ The complexity class $\mathrm{P}_{\|}^{\mathrm{NP}}$ is defined in terms of Turing machine having access to an oracle; oracle are artificial devices that can solve a problem in constant time, thus hiding part of the complexity of the overall problem. The class $\mathrm{P}^{\mathrm{NP}}$ is the class of problems that can be solved in polynomial time by a deterministic Turing machine which has access to an oracle for solving NP problems. The class $\mathrm{P}_{\|}^{\mathrm{NP}}$ is the subclass where, instead of asking a sequence of (dependent) queries to the oracle, the Turing machine is only allowed to ask one set of queries. We refer to [36, 44] for more details.

${ }^{2}$ The (quantitative) payoff for player $i$ can be expressed by payoff ${ }_{i}=\frac{R}{\text { power }_{i}}\left(1-e^{-0.5 \gamma_{i}}\right)^{L}$ where $\gamma_{i}$ is the signal-to-interference-and-noise ratio for player $i, R$ is the rate at which the wireless system transmits the information in bits per seconds and $L$ is the size of the packets in bits ([37).
} 


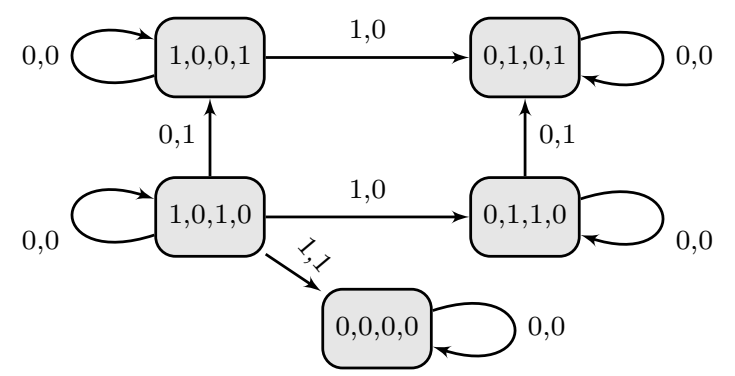

FIGURE 2. A simple game-model for the medium access control

to a wireless channel. During each slot, they can choose to either transmit or wait for the next slot. If too many users are emitting in the same slot, then they fail to send data. Each attempt to transmit costs energy to the players. They have to maximise the number of successful attempts using the energy available to them. We give in Figure 2 a possible model for that protocol for two players and at most one attempt per player and a congestion of 2 (that is, the two players should not transmit at the same time): each state is labelled with the energy level of the two players, and the number of successful attempts of each of the player. There is several Nash equilibria, and they give payoff 1 to every player: it consists in going to state $(0,1,0,1)$ by not simultaneously transmitting.

Related work. Game theory has been a very active area since the 1940's, with the pioneering works of Von Neumann, Morgenstern [43, Nash [35] and Shapley [38. It has had numerous uses in various domains, ranging from economics to human sciences and logic. Equilibria are a central concept in (non-zero-sum) games, as they are meant to represent rational behaviours of the players. Many important results about existence of various kinds of equilibria in different kinds of games have been established [43, 35, 20].

For applications in logic and computer science, games played on graphs have received more focus; also, computer scientists have been mostly looking for algorithmic solutions for deciding the existence and effectively computing equilibria and $\epsilon$-equilibria [15, 10, 40].

For two-player concurrent games with Büchi objectives, the existence of $\epsilon$-equilibria (in randomised strategies) was proved by Chatterjee [10]. However, exact Nash equilibria need not exist; turn-based games with Büchi objectives are an important subclass where Nash equilibria (even in pure strategies) always exist [15. When they exist, Nash equilibria need not be unique; equilibria where all the players lose can coexist with equilibria where some (or all) of them win. Ummels introduced constrained Nash equilibria, i.e., Nash equilibria where some players are required to win. In particular, he showed that the existence of constrained Nash equilibria can be decided in polynomial time for turn-based games with Büchi objectives [40]. In this paper, we extend this result to concurrent games, and to various classes of $\omega$-regular winning objectives. For concurrent games with $\omega$-regular objectives, the decidability of the constrained Nash equilibrium existence problem w.r.t. pure strategies was established by Fisman et al. [21, but their algorithm runs in doubly exponential time, whereas our algorithm runs in exponential time for objectives given as Büchi automata. Finally, Ummels and Wojtczak [42] proved that the existence of a Nash equilibrium in pure or randomised strategies is undecidable for stochastic games with reachability or Büchi objectives, which justifies our restriction to concurrent games without probabilistic 
transitions. They also proved a similar undecidability result for randomised Nash equilibria in non-stochastic games [41, hence we consider only pure-strategy Nash equilibria.

Several solution concepts have been defined and studied for games on graphs. In particular, secure equilibria [11, 17] are Nash equilibria where besides satisfying their primary objectives, the players try to prevent the other players from achieving their own (primary) objectives. Notice that our results in Sect. 6.4 and Sect. 7.1 do apply to such kinds of lexicographic combination of several objectives.

Temporal logics can also be used to express properties of games. While ATL [2] can mainly express only zero-sum properties, other logics such as ATL with strategy contexts $\left(\mathrm{ATL}_{\mathrm{sc}}\right)$ [16] and Strategy Logic (SL) [14, 33. can be used to express rich properties in a non-zero-sum setting. In terms of complexity however, model checking for such logics has high complexity: Nash equilibria can be expressed using one quantifier alternation (an existential quantification over strategy profiles followed with a universal quantification over deviations); model checking this fragment of $A T L_{s c}$ or $S L$ is 2-EXPTIME-complete.

\section{Definitions}

2.1. General definitions. In this section, we fix some definitions and notations.

Preorders. We fix a non-empty set $P$. A preorder over $P$ is a binary relation $\lesssim \subseteq P \times P$ that is reflexive and transitive. With a preorder $\lesssim$, we associate an equivalence relation $\sim$ defined so that $a \sim b$ if, and only if, $a \lesssim b$ and $b \lesssim a$. The equivalence class of $a$, written $[a]_{\lesssim}$, is the set $\{b \in P \mid a \sim b\}$. We also associate with $\lesssim$ a strict partial order $\prec$ defined so that $a \prec b$ if, and only if, $a \lesssim b$ and $b \underset{z}{a}$. A preorder $\lesssim$ is said total if, for all elements $a, b \in P$, either $a \lesssim b$, or $b \lesssim a$. An element $a$ in a subset $P^{\prime} \subseteq P$ is said maximal in $P^{\prime}$ if there is no $b \in P^{\prime}$ such that $a \prec b$; it is said minimal in $P^{\prime}$ if there is no $b \in P^{\prime}$ such that $b \prec a$. A preorder is said Noetherian (or upwards well-founded) if any subset $P^{\prime} \subseteq P$ has at least one maximal element. It is said almost-well-founded if any lower-bounded subset $P^{\prime} \subseteq P$ has a minimal element.

Transition systems. A transition system is a pair $\mathcal{S}=\langle$ States, Edg $\rangle$ where States is a set of states and $\operatorname{Edg} \subseteq$ States $\times$ States is the set of transitions. A path $\pi$ in $\mathcal{S}$ is a sequence $\left(s_{i}\right)_{0 \leq i<n}$ (where $n \in \mathbb{N}^{+} \cup\{\infty\}$ ) of states such that $\left(s_{i}, s_{i+1}\right) \in$ Edg for all $i \leq n$. The length of $\pi$, denoted by $|\pi|$, is $n-1$. The set of finite paths (also called histories) of $\mathcal{S}$ is denoted

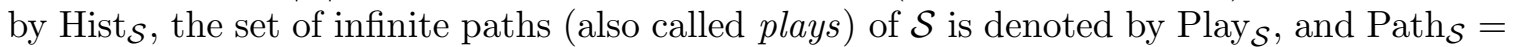
Hist $_{\mathcal{S}} \cup$ Play $_{\mathcal{S}}$ is the set of all paths of $\mathcal{S}$. Given a path $\pi=\left(s_{i}\right)_{0 \leq i<n}$ and an integer $j<n$, the $j$-th prefix (resp. $j$-th suffix, $j$-th state) of $\pi$, denoted by $\pi_{\leq j}$ (resp. $\pi_{\geq j}, \pi_{=j}$ ), is the finite path $\left(s_{i}\right)_{0 \leq i<j+1}$ (resp. the path $\left(s_{j+i}\right)_{0 \leq i<n-j}$, the state $\left.s_{j}\right)$. If $\pi=\left(s_{i}\right)_{0 \leq i<n}$ is a history, we write last $(\pi)=s_{|\pi|}$ for the last state of $\pi$. If $\pi^{\prime}$ is a path such that (last $\left.(\pi), \pi_{=0}^{\prime}\right) \in \operatorname{Edg}$, then the concatenation $\pi \cdot \pi^{\prime}$ is the path $\rho$ s.t. $\rho_{=i}=\pi_{=i}$ for $i \leq|\pi|$ and $\rho_{=i}=\pi_{=(i-1-|\pi|)}^{\prime}$ for $i>|\pi|$. In the sequel, we write $\operatorname{Hist}_{\mathcal{S}}(s), \operatorname{Play}_{\mathcal{S}}(s)$ and $\operatorname{Path}_{\mathcal{S}}(s)$ for the respective subsets of paths starting in state $s$. If $\pi$ is a play, $\operatorname{Occ}(\pi)=\left\{s \mid \exists j . \pi_{=j}=s\right\}$ is the sets of states that appears at least once along $\pi$ and $\operatorname{Inf}(\pi)=\left\{s \mid \forall i . \exists j \geq i . \pi_{=j}=s\right\}$ is the set of states that appears infinitely often along $\pi$. 
2.2. Concurrent games. Our definition of concurrent games extends the definition in [2] by allowing for more than two players, each of them having a preorder over plays.

Definition 2.1. A concurrent game is a tuple $\mathcal{G}=$ $\left\langle\right.$ States, Agt, Act, Mov, Tab, $\left.\left(\precsim_{A}\right)_{A \in \text { Agt }}\right\rangle$, where States is a finite non-empty set of states, Agt is a finite set of players, Act is a finite set of actions, and

- Mov: States $\times$ Agt $\rightarrow 2^{\text {Act }} \backslash\{\varnothing\}$ is a mapping indicating the actions available to a given player in a given state;

- Tab: States $\times$ Act $^{\text {Agt }} \rightarrow$ States associates, with a given state and a given move of the players (i.e., an element of Act ${ }^{\text {Agt }}$ ), the state resulting from that move;

- for each $A \in \mathrm{Agt}, \precsim A$ is a preorder over States $^{\omega}$, called the preference relation of player $A$.

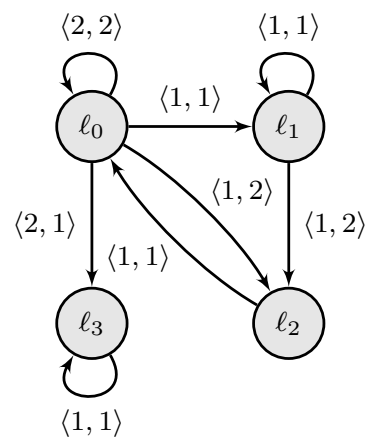

FiguRE 3. Representation of a two-player concurrent game

Figure 3 displays an example of a finite concurrent game. Transitions are labelled with the moves that trigger them. We say that a move $m_{\mathrm{Agt}}=\left\langle m_{A}\right\rangle_{A \in \mathrm{Agt}} \in$ Act $^{\text {Agt }}$ is legal at $s$ if $m_{A} \in \operatorname{Mov}(s, A)$ for all $A \in \operatorname{Agt}$. A game is turn-based if for each state the set of allowed moves is a singleton for all but at most one player.

In a concurrent game $\mathcal{G}$, whenever we arrive at a state $s$, the players simultaneously select an available action, which results in a legal move $m_{\text {Agt }}$; the next state of the game is then $\operatorname{Tab}\left(s, m_{\mathrm{Agt}}\right)$. The same process repeats ad infinitum to form an infinite sequence of states.

In the sequel, as no ambiguity will arise, we may abusively write $\mathcal{G}$ for its underlying transition system (States, $\operatorname{Edg}$ ) where $\operatorname{Edg}=\left\{\left(s, s^{\prime}\right) \in\right.$ States $\times$ States $\mid \exists m_{\text {Agt }} \in$ $\prod_{A \in \text { Agt }} \operatorname{Mov}(s, A)$ s.t. $\left.\operatorname{Tab}\left(s, m_{\text {Agt }}\right)=s^{\prime}\right\}$. The notions of paths and related concepts in concurrent games follow from this identification.

Remark 2.2 (Representation of finite games). In this paper, for finite games, we will assume an explicit encoding of the transition function Tab. Hence, its size, denoted |Tab|, is equal to $\sum_{s \in \text { States }} \prod_{A \in \text { Agt }}|\operatorname{Mov}(s, A)| \cdot\lceil\log (|\operatorname{States}|)\rceil$. Note that it can be exponential with respect to the number of players. A symbolic encoding of the transition table has been proposed in [30, in the setting of ATL model checking. This makes the problem harder, as the input is more succinct (see Remark 5.1 and Proposition 5.2 for a formal statement). We would also have a blowup in our setting, and prefer to keep the explicit representation in order to be able to compare with existing results. Notice that, as a matter of fact, there is no way to systematically avoid an explosion: as there are $\mid$ States $\mid$ States $|\cdot|$ Act $\left.\right|^{\mid \text {Agt } \mid}$ possible transition functions, for any encoding there is one function whose encoding will have size at least $\lceil\log (\mid$ States $\mid)\rangle \cdot \mid$ States $|\cdot|$ Act $\left.\right|^{\mid \text {Agt } \mid}$. The total size of the game, is then

$|\mathcal{G}|=\mid$ States $|+|$ States $|\cdot|$ Agt $|\cdot|$ Act $\left|+\sum_{s \in \text { States }} \prod_{A \in \text { Agt }}\right| \operatorname{Mov}(s, A)\left|\cdot\lceil\log (|\operatorname{States}|)\rceil+\sum_{A \in \text { Agt }}\right| \lesssim A \mid \cdot$

The size of a preference relation $\lesssim_{A}$ will depend on how it is encoded, and we will make it precise when it is relevant. This is given in Section 2.5.

Definition 2.3. Let $\mathcal{G}$ be a concurrent game, and $A \in$ Agt. A strategy for $A$ is a mapping $\sigma_{A}:$ Hist $_{\mathcal{G}} \rightarrow$ Act such that $\sigma_{A}(\pi) \in \operatorname{Mov}(\operatorname{last}(\pi), A)$ for all $\pi \in$ Hist $_{\mathcal{G}}$. A strategy $\sigma_{P}$ for a coalition $P \subseteq$ Agt is a tuple of strategies, one for each player in $P$. We write $\sigma_{P}=\left(\sigma_{A}\right)_{A \in P}$ 
for such a strategy. A strategy profile is a strategy for Agt. We write $\operatorname{Strat}_{\mathcal{G}}^{P}$ for the set of strategies of coalition $P$, and $\operatorname{Prof}_{\mathcal{G}}=$ Strat $_{\mathcal{G}}^{\mathrm{Agt}}$.

Note that, in this paper, we only consider pure (i.e., non-randomised) strategies. This is actually crucial in all the constructions we give (lasso representation in Subsection 3.1 and suspect-game construction in Section 4). Notice also that our strategies are based on the sequences of visited states (they map sequences of states to actions), which is realistic when considering multi-agent systems. In some settings, it is more usual to base strategies on the sequences of actions played by all the players. When dealing with Nash equilibria, this makes a big difference: strategies based on actions can immediately detect which player(s) deviated from their strategy; strategies based on states will only detect deviations because an unexpected state is visited, without knowing which player(s) is responsible for the deviation. Our construction precisely amounts to keeping track of a list of suspects for some deviation.

Let $\mathcal{G}$ be a game, $P$ a coalition, and $\sigma_{P}$ a strategy for $P$. A path $\pi$ is compatible with the strategy $\sigma_{P}$ if, for all $k<|\pi|$, there exists a move $m_{\text {Agt }}$ such that

(1) $m_{\text {Agt }}$ is legal at $\pi_{=k}$,

(2) $m_{A}=\sigma_{A}\left(\pi_{\leq k}\right)$ for all $A \in P$, and

(3) $\operatorname{Tab}\left(\pi_{=k}, m_{\mathrm{Agt}}\right)=\pi_{=k+1}$.

We write $\operatorname{Out}_{\mathcal{G}}\left(\sigma_{P}\right)$ for the set of paths (called the outcomes) in $\mathcal{G}$ that are compatible with strategy $\sigma_{P}$ of $P$. We write $\mathrm{Out}_{\mathcal{G}}^{\mathrm{f}}$ (resp. Out ${ }_{\mathcal{G}}^{\infty}$ ) for the finite (resp. infinite) outcomes, and $\operatorname{Out}_{\mathcal{G}}\left(s, \sigma_{P}\right), \operatorname{Out}_{\mathcal{G}}^{\mathrm{f}}\left(s, \sigma_{P}\right)$ and $\operatorname{Out}_{\mathcal{G}}^{\infty}\left(s, \sigma_{P}\right)$ for the respective sets of outcomes of $\sigma_{P}$ with initial state $s$. Notice that any strategy profile has a single infinite outcome from a given state. In the sequel, when given a strategy profile $\sigma_{\text {Agt }}$, we identify $\operatorname{Out}\left(s, \sigma_{\text {Agt }}\right)$ with the unique play it contains.

A concurrent game involving only two players ( $A$ and $B$, say) is zero-sum if, for any two plays $\pi$ and $\pi^{\prime}$, it holds $\pi \precsim_{A} \pi^{\prime}$ if, and only if, $\pi^{\prime} \precsim_{B} \pi$. Such a setting is purely antagonistic, as both players have opposite objectives. The most relevant concept in such a setting is that of winning strategies, where the aim is for one player to achieve her objectives whatever the other players do. In non-zero-sum games, winning strategies are usually too restricted, and the most relevant concepts are equilibria, which correspond to strategies that satisfy (which can be given several meanings) all the players. One of the most studied notion of equilibria is Nash equilibria [35], which we now introduce.

2.3. Nash equilibria. We begin with introducing some vocabulary. When $\pi \precsim_{A} \pi^{\prime}$, we say that $\pi^{\prime}$ is at least as good as $\pi$ for $A$. We say that a strategy $\sigma_{A}$ for $A$ ensures $\pi$ if every outcome of $\sigma_{A}$ is at least as good as $\pi$ for $A$, and that $A$ can ensure $\pi$ when such a strategy exists.

Given a move $m_{\mathrm{Agt}}$ and an action $m^{\prime}$ for some player $A$, we write $m_{\mathrm{Agt}}\left[A \mapsto m^{\prime}\right]$ for the move $n_{\text {Agt }}$ with $n_{B}=m_{B}$ when $B \neq A$ and $n_{A}=m^{\prime}$. This is extended to strategies in the natural way.

Definition 2.4. Let $\mathcal{G}$ be a concurrent game and let $s$ be a state of $\mathcal{G}$. A Nash equilibrium of $\mathcal{G}$ from $s$ is a strategy profile $\sigma_{\mathrm{Agt}} \in \operatorname{Prof}_{\mathcal{G}}$ such that $\operatorname{Out}\left(s, \sigma_{\mathrm{Agt}}\left[A \mapsto \sigma^{\prime}\right]\right) \precsim{ }_{A} \operatorname{Out}\left(s, \sigma_{\mathrm{Agt}}\right)$ for all players $A \in$ Agt and all strategies $\sigma^{\prime} \in \operatorname{Strat}^{A}$. 
So, Nash equilibria are strategy profiles where no single player has an incentive to unilaterally deviate from her strategy.

Remark 2.5. Our definition of a Nash equilibrium requires any deviation to be worse or equivalent to the equilibrium. Another possible definition would have been to ask any deviation to be no better than the equilibrium. Those two definitions yield different notions of Nash equilibria (unless the preorders are total), as illustrated in Figure 4. the black node $n$ represents $\operatorname{Out}\left(s, \sigma_{\text {Agt }}\right)$, the light-gray area contains the nodes $n^{\prime}$ such that $n^{\prime} \precsim n$, while the dark-gray area contains the nodes $n^{\prime}$ for which $n \not ू n^{\prime}$.

This alternative definition would also be meaningful,

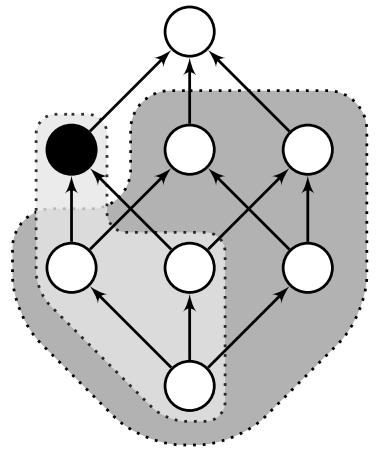

Figure 4. Two different notions of improvements for a nontotal order. and the techniques we develop in this paper could be adapted to handle such a variant.

In this paper we will give a general construction that relates Nash equilibria in a game (which can be infinite) and winning strategies in a two-player turn-based game (called the suspect game), it is presented in Section 4. We will then be mostly interested in solving the decision problems that we define next, when games are finite.

2.4. Decision problems we will consider. Given a concurrent game $\mathcal{G}=\langle$ States, Agt, Act, Mov, Tab, $\left.\left(\precsim_{A}\right)_{A \in \text { Agt }}\right\rangle$ and a state $s \in$ States, we consider the following problems:

- Value problem: Given a player $A$ and a play $\pi$, is there a strategy $\sigma_{A}$ for player $A$ such that for any outcome $\rho$ in $\mathcal{G}$ from $s$ of $\sigma_{A}$, it holds $\pi \precsim_{A} \rho$ ?

- $N E$ Existence problem: Does there exist a Nash equilibrium in $\mathcal{G}$ from $s$ ?

- Constrained $N E$ existence problem: Given two plays $\pi_{A}^{-}$and $\pi_{A}^{+}$for each player $A$, does there exist a Nash equilibrium in $\mathcal{G}$ from $s$ whose outcome $\pi$ satisfies $\pi_{A}^{-} \precsim_{A} \pi_{{ }_{A}} \pi_{A}^{+}$for all $A \in$ Agt?

We will focus on decidability and complexity results of these three problems when games are finite, for various classes of preference relations. Complexity results will heavily rely on what preorders we allow for the preference relation and how they are represented. We have already discussed the representation of the game structure in Remark 2.2. We define and discuss now the various preference relations we will study, and explain how we encode the various inputs to the problems.

2.5. Focus on the preference relations we will consider. We define the various classes of preference relations we will focus on in the rest of the paper. We begin with singleobjective preference relations, and we then define a more general class of ordered objectives. We fix a game $\mathcal{G}=\left\langle\right.$ States, Agt, Act, Mov, Tab, $\left.\left(\precsim_{A}\right)_{A \in \text { Agt }}\right\rangle$. 


\subsubsection{Single-objective preference relations.}

Definition 2.6. An objective (or winning condition) is an arbitrary set of plays. A preference relation $\precsim_{A}$ is single-objective whenever there exists an objective $\Omega_{A}$ such that: $\rho \precsim A \rho^{\prime}$ if, and only if, $\rho^{\prime} \in \Omega_{A}$ (we then say that $\rho^{\prime}$ is winning for $A$ ) or $\rho \notin \Omega_{A}$ (we then say that $\rho$ is losing for $A$ ).

The setting of single-objective preference relations is purely qualitative, since a player can only win (in case the outcome is in her objective), or lose (otherwise).

An objective $\Omega$ can be specified in various ways. Next we will consider the following families of $\omega$-regular objectives:

- A reachability objective is given by a target set $T \subseteq$ States and the corresponding set of winning plays is defined by

$$
\Omega_{T}^{\text {Reach }}=\{\rho \in \text { Play } \mid \operatorname{Occ}(\rho) \cap T \neq \varnothing\} .
$$

- A safety objective is given by a target set $T \subseteq$ States and the corresponding set of winning plays is defined by

$$
\Omega_{T}^{\text {Safety }}=\{\rho \in \text { Play } \mid \operatorname{Occ}(\rho) \cap T=\varnothing\} .
$$

- A Büchi objective is given by a target set $T \subseteq$ States and the corresponding set of winning plays is defined by

$$
\Omega_{T}^{\text {Büchi }}=\{\rho \in \text { Play } \mid \operatorname{Inf}(\rho) \cap T \neq \varnothing\} .
$$

- A co-Büchi objective is given by a target set $T \subseteq$ States and the corresponding set of winning plays is defined by

$$
\Omega_{T}^{\text {co-Büchi }}=\{\rho \in \text { Play } \mid \operatorname{Inf}(\rho) \cap T=\varnothing\} .
$$

- A parity objective is given by a priority function $p$ : States $\mapsto \llbracket 0, d \rrbracket$ (where $\llbracket 0, d \rrbracket=$ $[0, d] \cap \mathbb{Z})$ with $d \in \mathbb{N}$, and the corresponding set of winning plays is defined by

$$
\Omega_{p}^{\text {Parity }}=\{\rho \in \text { Play } \mid \min (\operatorname{Inf}(p(\rho))) \text { is even }\} .
$$

- A Streett objective is given by a tuple $\left(Q_{i}, R_{i}\right)_{i \in \llbracket 1, k \rrbracket}$ and the corresponding set of winning plays is defined by

$$
\Omega_{\left(Q_{i}, R_{i}\right)_{i \in \llbracket 1, k \rrbracket}}^{\text {Street }}=\left\{\rho \in \text { Play } \mid \forall i . \operatorname{Inf}(\rho) \cap Q_{i} \neq \varnothing \Rightarrow \operatorname{Inf}(\rho) \cap R_{i} \neq \varnothing\right\} .
$$

- A Rabin objective is given by a tuple $\left(Q_{i}, R_{i}\right)_{i \in \llbracket 1, k \rrbracket}$ and the corresponding set of winning plays is defined by

$$
\Omega_{\left(Q_{i}, R_{i}\right)_{i \in \mathbb{1}, k \rrbracket}}^{\text {Rabin }}=\left\{\rho \in \text { Play } \mid \exists i . \operatorname{Inf}(\rho) \cap Q_{i} \neq \varnothing \wedge \operatorname{Inf}(\rho) \cap R_{i}=\varnothing\right\} .
$$

- A Muller objective is given by a finite set $C$, a coloring function $c$ : States $\mapsto C$, and a set $\mathcal{F} \subseteq 2^{C}$. The corresponding set of winning plays is then defined by

$$
\Omega_{c, \mathcal{F}}^{\mathrm{Muller}}=\{\rho \in \text { Play } \mid \operatorname{Inf}(c(\rho)) \in \mathcal{F}\} .
$$

We will also consider the following other types of objectives: 


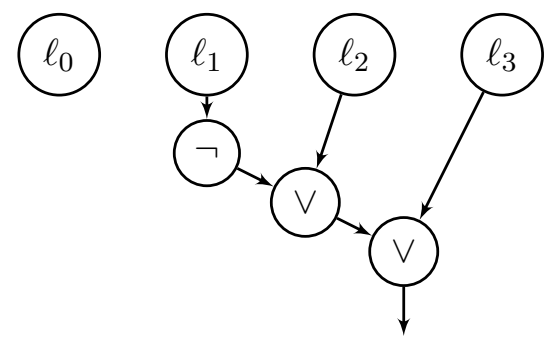

Figure 5. Boolean circuit defining the condition that either $\ell_{3}$ appears infinitely often, or if $\ell_{1}$ appears infinitely often then so does $\ell_{2}$.

- A circuit objective is given by a boolean circuit $C$ with the set States as input nodes and one output node. A play $\rho$ is winning if and only if $C$ evaluates to true when the input nodes corresponding to states in $\operatorname{Inf}(\rho)$ are set to true, and all other input nodes are set to false. We write $\Omega_{C}^{\text {Circuit }}$ for the set of winning plays.

Figure 5 displays an example of a circuit for the game of Figure 3 , this Boolean circuit defines the condition that either $\ell_{3}$ appears infinitely often, or if $\ell_{1}$ appears infinitely often then so does $\ell_{2}$.

- A deterministic Büchi automaton objective is given by a deterministic Büchi automaton $\mathcal{A}=\left\langle Q, \Sigma, \delta, q_{0}, R\right\rangle$, with $\Sigma=$ States. Then the corresponding set of winning plays is defined by

$$
\Omega_{\mathcal{A}}^{\text {det-Büchi-aut }}=\mathcal{L}(\mathcal{A}) .
$$

- A deterministic Rabin automaton objective is given by a deterministic Rabin automaton $\mathcal{A}=\left\langle Q, \Sigma, \delta, q_{0},\left(E_{i}, F_{i}\right)_{i \in \llbracket 1, k \rrbracket}\right\rangle$, with $\Sigma=$ States. Then the corresponding set of winning plays is defined by

$$
\Omega_{\mathcal{A}}^{\text {det-Rabin-aut }}=\mathcal{L}(\mathcal{A})
$$

- A Presburger-definable objective is given by a Presburger formula $\phi$ with free variables $\left(X_{s}\right)_{s \in \text { States. }}$. The corresponding set of winning plays is defined by

$$
\left.\Omega_{\phi}^{\text {Presb }}=\left\{\rho \in \text { Play } \mid \phi(\# s(\rho))_{s \in \text { States }}\right)=0\right\}
$$

where $\# s(\rho)$ is the number of occurrences 3 of state $s$ along $\rho$.

Encodings. For complexity issues we now make explicit how the various objectives are encoded:

- Reachability, safety, Büchi and co-Büchi objectives are given by a set $T \subseteq$ States, they can therefore be encoded using |States| bits.

- For parity objectives, we assume without loss of generality that $d \leq 2 \cdot \mid$ States $\mid$. The priority function has then size at most $\mid$ States $\mid \cdot\lceil\log (2 \cdot \mid$ States $\mid+1)\rceil$.

- Street and Rabin objectives are given by tuples $\left(Q_{i}, R_{i}\right)_{i \in \llbracket 1, k \rrbracket}$. Their sizes are given by: $\sum_{i \in \llbracket 1, k \rrbracket}\left|Q_{i}\right|\lceil\log (\mid$ States $\mid)\rceil$.

- Muller objectives are given by a coloring function and a set $\mathcal{F}$. Its size is $\mid$ States $\mid$. $\lceil\log (|C|)\rceil+|\mathcal{F}| \cdot\lceil\log (|C|)\rceil$. Note that thanks to the coloring function, this encoding can be exponentially more succinct than an explicit representation such as the one considered in [26].

\footnotetext{
${ }^{3}$ By convention, if $s \in \operatorname{Inf}(\rho)$, and variable $X_{s}$ appears in $\phi$, then $\rho \notin \Omega_{\phi}^{\text {Presb }}$.
} 


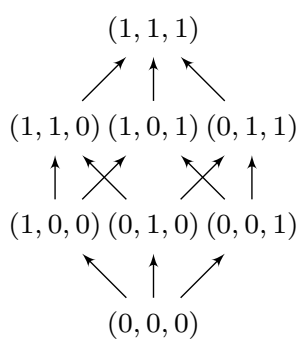

A. Subset preorder

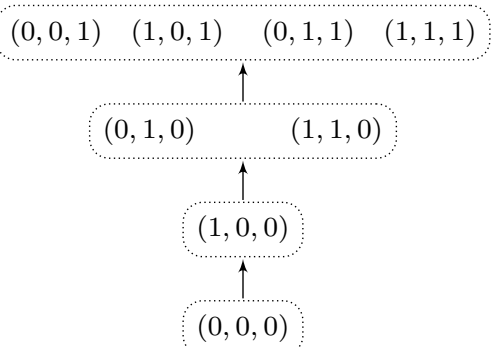

B. Maximise preorder

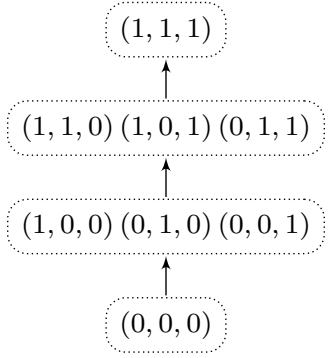

C. Counting preorder

$(0,0,0) \rightarrow(0,0,1) \rightarrow(0,1,0) \rightarrow(0,1,1) \rightarrow(1,0,0) \rightarrow(1,0,1) \rightarrow(1,1,0) \rightarrow(1,1,1)$

D. Lexicographic order

FiguRE 6. Examples of preorders (for $n=3$ ): dotted boxes represent equivalence classes for the relation $\sim$, defined as $a \sim b \Leftrightarrow a \lesssim b \wedge b \lesssim a$; arrows represent the preorder relation $\lesssim$ quotiented by $\sim$.

- The size of objectives given by circuits, deterministic automata or Presburger formulas is that of the corresponding circuits, deterministic automata or Presburger formulas.

Encodings of thresholds in inputs of the value and the constrained NE existence problems. For all the objectives except for those given by automata, whether a play $\rho$ satisfies the objective or not only depends on the $\operatorname{sets} \operatorname{Occ}(\rho)$ and $\operatorname{Inf}(\rho)$. The various thresholds will therefore be encoded as such pairs (Occ, Inf).

For deterministic-automata objectives, the thresholds will be also encoded as pairs of sets of states of the objectives, representing respectively the set of states which are visited and the set of states which are visited infinitely often.

For the Boolean circuit objectives, whether a play $\rho$ satisfies the objective or not only depends on the set $\operatorname{Inf}(\rho)$. Therefore we will use as encoding for the threshold a single set Inf.

For the Presburger formulas objectives, we will use as encoding for the thresholds the Parikh image of the play (i.e., the number of visits to each of the states).

2.5.2. Ordered objectives. We now turn to a more general class of preference relations, allowing for a semi-quantitative setting.

Definition 2.7. An ordered objective is a pair $\omega=\left\langle\left(\Omega_{i}\right)_{1 \leq i \leq n}, \lesssim\right\rangle$, where, for every $1 \leq i \leq$ $n, \Omega_{i}$ is an objective, and $\lesssim$ is a preorder on $\{0,1\}^{n}$. A play $\rho$ is assigned a payoff vector w.r.t. that ordered objective, which is defined as payoff $\omega(\rho)=\mathbf{1}_{\left\{i \mid \rho \in \Omega_{i}\right\}} \in\{0,1\}^{n}$ (where $\mathbf{1}_{S}$ is the vector $v$ such that $\left.v_{i}=1 \Leftrightarrow i \in S\right)$. The corresponding preference relation $\precsim \omega$ is then defined by $\rho \precsim \omega \rho^{\prime}$ if, and only if, $\operatorname{payoff}_{\omega}(\rho) \lesssim \operatorname{payoff}_{\omega}\left(\rho^{\prime}\right)$.

There are many ways of specifying a preorder. We define below the preorders on $\{0,1\}^{n}$ that we consider in the sequel. Figure 6 displays four such preorders for $n=3$. For the purpose of these definitions, we assume that $\max \varnothing=-\infty$. 
(1) Conjunction: $v \lesssim w$ if, and only if, either $v_{i}=0$ for some $1 \leq i \leq n$, or $w_{i}=1$ for all $1 \leq i \leq n$. This corresponds to the case where a player wants to achieve all her objectives.

(2) Disjunction: $v \lesssim w$ if, and only if, either $v_{i}=0$ for all $1 \leq i \leq n$, or $w_{i}=1$ for some $1 \leq i \leq n$. The aim here is to satisfy at least one objective.

(3) Counting: $v \lesssim w$ if, and only if, $\left|\left\{i \mid v_{i}=1\right\}\right| \leq\left|\left\{i \mid w_{i}=1\right\}\right|$. The aim is to maximise the number of conditions that are satisfied;

(4) Subset: $v \lesssim w$ if, and only if, $\left\{i \mid v_{i}=1\right\} \subseteq\left\{i \mid w_{i}=1\right\}$ : in this setting, a player will always struggle to satisfy a larger (for inclusion) set of objectives.

(5) Maximise: $v \lesssim w$ if, and only if, $\max \left\{i \mid v_{i}=1\right\} \leq \max \left\{i \mid w_{i}=1\right\}$. The aim is to maximise the highest index of the objectives that are satisfied.

(6) Lexicographic: $v \lesssim w$ if, and only if, either $v=w$, or there is $1 \leq i \leq n$ such that $v_{i}=0, w_{i}=1$ and $v_{j}=w_{j}$ for all $1 \leq j<i$.

(7) Boolean Circuit: given a Boolean circuit, with input from $\{0,1\}^{2 n}, v \lesssim w$ if, and only if, the circuit evaluates 1 on input $v_{1} \ldots v_{n} w_{1} \ldots w_{n}$.

(8) Monotonic Boolean Circuit: same as above, with the restriction that the input gates corresponding to $v$ are negated, and no other negation appear in the circuit.

In terms of expressiveness, any preorder over $\{0,1\}^{n}$ can be given as a Boolean circuit: for each pair $(v, w)$ with $v \lesssim w$, it is possible to construct a circuit whose output is 1 if, and only if, the input is $v_{1} \ldots v_{n} w_{1} \ldots w_{n}$; taking the disjunction of all these circuits we obtain a Boolean circuit defining the preorder. Its size can be bounded by $2^{2 n+3} n$, which is exponential in general. But all the above examples ((1)-(6)) can be specified with a circuit of polynomial size. In Figure 7 we give a polynomial-size Boolean circuit for the subset preorder. In the following, for complexity issues, we will assume that the encoding of all preorders (1)-(6) takes constant size, and that the size of the preorder when it is given as a Boolean circuit is precisely the size of the circuit for input size $n$, where $n$ is the number of objectives.

A preorder $\lesssim$ is monotonic if it is compatible with the subset ordering, i.e. if $\left\{i \mid v_{i}=\right.$ $1\} \subseteq\left\{i \mid w_{i}=1\right\}$ implies $v \lesssim w$. Hence, a preorder is monotonic if fulfilling more objectives never results in a lower payoff. All our examples of preorders except for the Boolean circuit preorder are monotonic. Moreover, any monotonic preorder can be expressed as a monotonic Boolean circuit: for a pair $(v, w)$ with $v \lesssim w$, we can build a circuit whose output is 1 if, and only if, the input is $v_{1} \ldots v_{n} w_{1} \ldots w_{n}$. We can require this circuit to have negation at the leaves. Indeed, if the input $w_{j}$ appears negated, and if $w_{j}=0$, then by monotonicity, also the input $(v, \tilde{w})$ is accepted, with $\tilde{w}_{i}=w_{i}$ when $i \neq j$ and $\tilde{w}_{j}=1$. Hence the negated input gate can be replaced with true. Similarly for positive occurrences of any $v_{j}$. Hence any monotonic preorder can be written as a monotonic Boolean circuit. Notice that with Definition 2.4, any Nash equilibrium $\sigma_{\text {Agt }}$ for the subset preorder is also a Nash equilibrium for any monotonic preorder.

Next we will be be interested in two kinds of ordered objectives, ordered reachability objectives, where all objectives are supposed to be reachability objectives, and ordered Büchi objectives, where all objectives are supposed to be Büchi objectives. Note that other classical objectives (parity, Streett, Rabin, Muller, etc.) can be equivalently described with a preorder given by a polynomial-size Boolean circuit over Büchi objectives. For instance, each set of a Muller condition can be encoded as a conjunction of Büchi and co-Büchi conditions. 


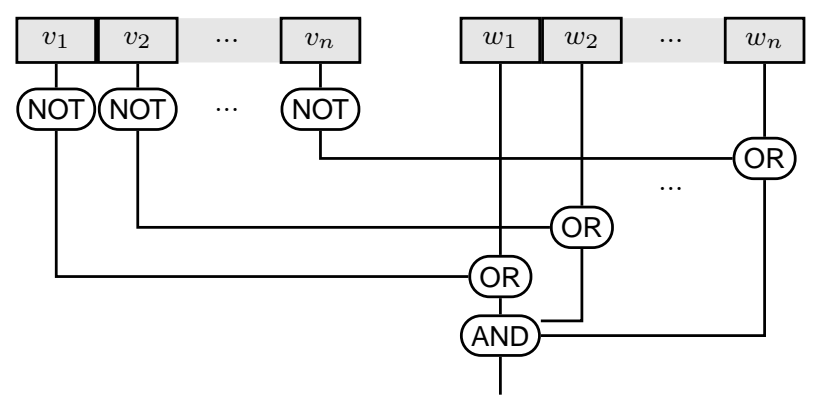

FigURE 7. Boolean circuit defining the subset preorder

For ordered reachability (resp. Büchi) objectives, thresholds used as inputs to the various decision problems will be given by the set of states that are visited (resp. visited infinitely often).

In Sections 6 and 7 , we will be interested in games where, for every player $A$, the preference relation $\precsim_{A}$ is given by an ordered objective $\omega_{A}=\left\langle\left(\Omega_{i}^{A}\right)_{1 \leq i \leq n_{A}}, \lesssim_{A}\right\rangle$. We will then write payoff $A$ instead of payoff $\omega_{A}$ for the payoffs, and if $\rho$ is a play, $\operatorname{payoff}(\rho)=$ (payoff $\left._{A}(\rho)\right)_{A \in \mathrm{Agt}}$.

\subsection{Undecidability of all three problems for single Presburger-definable objec-} tives. We end this section with an undecidability result in the quite general setting of Presburger-definable preference relations.

Theorem 2.8. The value, NE existence and constrained NE existence problems are undecidable for finite games with preference relations given by Presburger-definable qualitative objectives.

Proof. We first prove the result for the constrained NE existence problem, by encoding a two-counter machine. We fix a two-counter machine, and assume without loss of generality that the halting state is preceded by a non-zero test for the two counters (hence if the machine halts, the two counters have a positive value in the halting state).

We begin with defining a family of preorders. Fix two sets of states $S$ and $T$; a play is said $(S=T)$-winning if the number of visits to $S$ equals the number of visits to $T$, and both are finite. Formally, $\pi \precsim_{S=T} \pi^{\prime}$ whenever $\pi$ is not $(S=T)$-winning, or $\pi^{\prime}$ is.

We use such preorders to encode the acceptance problem for two-counter machines: the value of counter $c_{1}$ is encoded as the difference between the number of visits to $S_{1}$ and $T_{1}$, and similarly for counter $c_{2}$. Incrementing counter $c_{i}$ thus consists in visiting a state in $S_{i}$, and decrementing consists in visiting $T_{i}$; in other terms, if instruction $q_{k}$ of the two-counter machine consists in incrementing $c_{1}$ and jumping to $q_{k^{\prime}}$, then the game will have a transition from some state $q_{k}$ to a state in $S_{1}$, and a transition from there to $q_{k^{\prime}}$. The game involves three players: $A_{1}, A_{2}$ and $B$. The aim of player $A_{1}$ (resp. $A_{2}$ ) is to visit $S_{1}$ and $T_{1}$ (resp. $S_{2}$ and $T_{2}$ ) the same number of times: player $A_{i}$ 's preference is $\precsim S_{i}=T_{i}$. The aim of player $B$ is to reach the state corresponding to the halting state of the two-counter machine. Due to the assumption on the two-counter machine, if $B$ wins, then both $A_{1}$ and $A_{2}$ lose. 
It remains to encode the zero-test: this is achieved by the module of Figure 8. In this module, player $B$ tries to avoid the three sink states (marked in grey), since this would prevent her from reaching her goal. When entering the module, player $B$ has to choose one of the available branches: if she decides to go to $u_{i}^{\neq 0}$, then $A_{i}$ could take the play into the self-loop, which is winning for her if $S_{i}$ and $T_{i}$ have been visited the same number of times in the history of this path, which corresponds to having $c_{i}=0$; hence player $B$ should play to $u_{i}^{\neq 0}$ only if $c_{i} \neq 0$, so that $A_{1}$ has no interest in going to this self-loop.

Similarly, if player $B$ decides to go to $u_{i}^{=0}$, player $A_{i}$ has the opportunity to "leave" the main stream of the game, and go to $s_{i}$ or $t_{i}$ (obviously

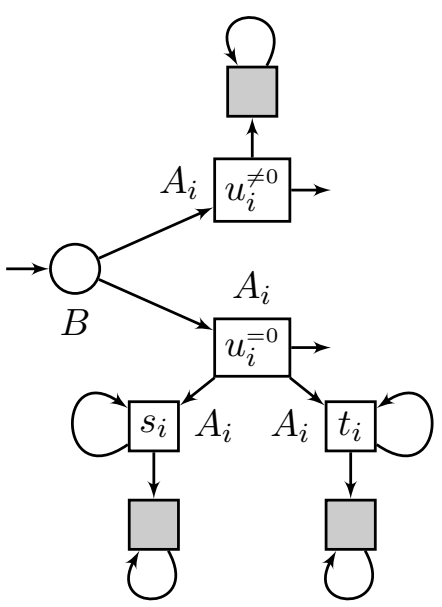

FiguRE 8. Testing whether $c_{i}=0$. $s_{i} \in S_{i}$ and $t_{i} \in T_{i}$ ). If the numbers of visits to $S_{i}$ and $T_{i}$ up to that point are different, then player $A_{i}$ has the opportunity to make both numbers equal, and to win. Conversely, if both numbers are equal (i.e., $c_{i}=0$ ), then going to $s_{i}$ or $t_{i}$ will be losing for $A_{i}$, whatever happens from there. Hence, if $c_{i}=0$ when entering the module, then player $B$ should go to $u_{i}^{=0}$.

One can then easily show that the two-counter machine stops if, and only if, there is a Nash equilibrium in the resulting game $\mathcal{G}$, in which player $B$ wins and players $A_{1}$ and $A_{2}$ lose. Indeed, assume that the machine stops, and consider the strategies where player $B$ plays (in the first state of the test modules) according to the value of the corresponding counter, and where players $A_{1}$ and $A_{2}$ always keep the play in the main stream of the game. Since the machine stops, player $B$ wins, while players $A_{1}$ and $A_{2}$ lose. Moreover, none of them has a way to improve their payoff: since player $B$ plays according to the values of the counters, players $A_{1}$ and $A_{2}$ would not benefit from deviating from their above strategies. Conversely, if there is such a Nash equilibrium, then in any visited test module, player $B$ always plays according to the values of the counters: otherwise, player $A_{1}$ (or $A_{2}$ ) would have the opportunity to win the game. By construction, this means that the run of the Nash equilibrium corresponds to the execution of the two-counter machine. As player $B$ wins, this execution reaches the halting state.

Finally, it is not difficult to adapt this reduction to involve only two players: players $A_{1}$ and $A_{2}$ would be replaced by one single player $A$, in charge of ensuring that both conditions (for $c_{1}$ and $c_{2}$ ) are fulfilled. This requires minor changes to the module for testing $c_{i}=0$ : when leaving the main stream of the game in a module for testing counter $c_{i}$, player $A$ should be given the opportunity (after the grey state) to visit states $S_{3-i}$ or $T_{3-i}$ in order to adjust that part of her objective.

By changing the winning condition for Player $B$, the game $\mathcal{G}$ can also be made zero-sum: for this, $B$ must lose if the play remains in the main stream forever without visiting the final state; otherwise, $B$ loses if the number of visits to $s_{i}$ and $t_{i}$ are finite and equal for both $i=1$ and $i=2 ; B$ wins in any other case. The objective of player $A$ is opposite. It is not difficult to modify the proof above for showing that the two-counter machine halts if, and only if, player $B$ has a winning strategy in this game. 
Finally, by adding a small initial module depicted on Figure 9 to this zero-sum version of the game $\mathcal{G}$, one can encode the halting problem for two-counter machines to the NE existence problem. Indeed, in the zero-sum game, there is exactly one Nash equilibrium, with only two possible payoffs (either $A$ wins, or $B$ wins). Now, assuming that $A$ loses and $B$ wins in state $s_{1}$, then there is a (pure) Nash equilibrium in the game extended with the initial module if, and only if, player $B$ wins in the zero-

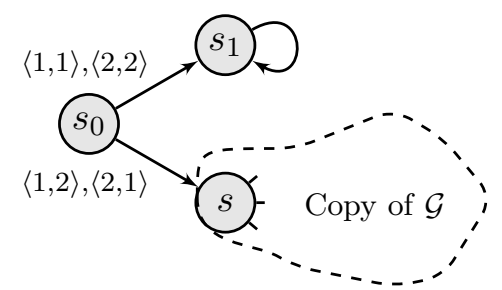

Figure 9. Extending the game with an initial concurrent module sum game above.

\section{Preliminary Results}

This section contains general results that will be applied later in various settings. In each of the statements, we give the restrictions on the games and on the preference relations that should be satisfied.

3.1. Nash equilibria as lasso runs. We first characterise outcomes of Nash equilibria as ultimately periodic runs, in the case where preference relations only depend on the set of states that are visited, and on the set of states that are visited infinitely often. Note that $\omega$-regular conditions satisfy this hypothesis, but Presburger relations such as the ones used for proving Theorem 2.8 do not.

Proposition 3.1. Let $\mathcal{G}=\left\langle\right.$ States, Agt, Act, Mov, Tab, $\left.\left(\precsim_{A}\right)_{A \in \mathrm{Agt}}\right\rangle$ be a finite concurrent game such that, for every player $A$, it hold $\S^{A} \sim_{A} \rho^{\prime}$ as soon as $\operatorname{Inf}(\rho)=\operatorname{Inf}\left(\rho^{\prime}\right)$ and $\operatorname{Occ}(\rho)=\operatorname{Occ}\left(\rho^{\prime}\right)$. Let $\rho \in$ Play. If there is a Nash equilibrium with outcome $\rho$, then there is a Nash equilibrium with outcome $\rho^{\prime}$ of the form $\pi \cdot \tau^{\omega}$ such that $\rho \sim_{A} \rho^{\prime}$, and where $|\pi|$ and $|\tau|$ are bounded by $\mid$ States $\left.\right|^{2}$.

Proof. Let $\sigma_{\text {Agt }}$ be a Nash equilibrium from some state $s$, and $\rho$ be its outcome. We define a new strategy profile $\sigma_{\text {Agt }}^{\prime}$, whose outcome from $s$ is ultimately periodic, and then show that $\sigma_{\text {Agt }}^{\prime}$ is a Nash equilibrium from $s$.

To begin with, we inductively construct a history $\pi=\pi_{0} \pi_{1} \ldots \pi_{n}$ that is not too long and visits precisely those states that are visited by $\rho$ (that is, $\operatorname{Occ}(\pi)=\operatorname{Occ}(\rho))$.

The initial state is $\pi_{0}=\rho_{0}=s$. Then we assume we have constructed $\pi_{\leq k}=\pi_{0} \ldots \pi_{k}$ which visits exactly the same states as $\rho_{\leq k^{\prime}}$ for some $k^{\prime}$. If all the states of $\rho$ have been visited in $\pi_{\leq k}$ then the construction is over. Otherwise there is an index $i$ such that $\rho_{i}$ does not appear in $\pi_{\leq k}$. We therefore define our next target as the smallest such $i$ : we let $t\left(\pi_{\leq k}\right)=\min \left\{i \mid \forall j \leq k . \pi_{j} \neq \rho_{i}\right\}$. We then look at the occurrence of the current state $\pi_{k}$ that is the closest to the target in $\rho$ : we let $c\left(\pi_{\leq k}\right)=\max \left\{j<t\left(\pi_{\leq k}\right) \mid \pi_{k}=\rho_{j}\right\}$. Then we emulate what happens at that position by choosing $\pi_{j+1}=\rho_{c\left(\pi_{\leq j}\right)+1}$. Then $\pi_{k+1}$ is either the target, or a state that has already been seen before in $\pi_{\leq k}$, in which case the resulting $\pi_{\leq k+1}$ visits exactly the same states as $\rho_{\leq c}\left(\pi_{\leq k}\right)+1$.

At each step, either the number of remaining targets strictly decreases, or the number of remaining targets is constant but the distance to the next target strictly decreases.

\footnotetext{
${ }^{4}$ We recall that $\rho \sim_{A} \rho^{\prime}$ if, and only if, $\rho \precsim A \rho^{\prime}$ and $\rho^{\prime} \precsim_{A} \rho$.
} 
Therefore the construction terminates. Moreover, notice that between two targets we do not visit the same state twice, and we visit only states that have already been visited, plus the target. As the number of targets is bounded by $\mid$ States $\mid$, we get that the length of the path $\pi$ constructed thus far is bounded by $1+\mid$ States $\mid \cdot(\mid$ States $\mid-1) / 2$.

Using similar ideas, we now inductively construct $\tau=\tau_{0} \tau_{1} \ldots \tau_{m}$, which visits precisely those states which are seen infinitely often along $\rho$, and which is not too long. Let $l$ be the least index after which the states visited by $\rho$ are visited infinitely often, i.e. $l=$ $\min \left\{i \in \mathbb{N} \mid \forall j \geq i . \rho_{j} \in \operatorname{Inf}(\rho)\right\}$. The run $\rho_{\geq l}$ is such that its set of visited states and its set of states visited infinitely often coincide. We therefore define $\tau$ in the same way we have defined $\pi$ above, but for play $\rho_{\geq l}$. As a by-product, we also get $c\left(\tau_{\leq k}\right)$, for $k<m$.

We now need to glue $\pi$ and $\tau$ together, and to ensure that $\tau$ can be glued to itself, so that $\pi \cdot \tau^{\omega}$ is a real run. We therefore need to link the last state of $\pi$ with the first state of $\tau$ (and similarly the last state of $\tau$ with its first state). This possibly requires appending some more states to $\pi$ and $\tau$ : we fix the target of $\pi$ and $\tau$ to be $\tau_{0}$, and apply the same construction as previously until the target is reached. The total length of the resulting paths $\pi^{\prime}$ and $\tau^{\prime}$ is bounded by $1+(\mid$ States $\mid-1) \cdot(\mid$ States $\mid+2) / 2$ which is less than $\mid$ States $\left.\right|^{2}$.

We let $\rho^{\prime}=\pi^{\prime} \cdot \tau^{\prime \omega}$, and abusively write $c\left(\rho_{\leq k}^{\prime}\right)$ for $c\left(\pi_{\leq k}^{\prime}\right)$ if $k \leq\left|\pi^{\prime}\right|$ and $c\left(\tau_{\leq k^{\prime}}^{\prime}\right)$ with $k^{\prime}=\left(k-1-\left|\pi^{\prime}\right|\right) \bmod \left|\tau^{\prime}\right|$ otherwise. We now define our new strategy profile, having $\rho^{\prime}$ as outcome from $s$. Given a history $h$ :

- if $h$ followed the expected path, i.e., $h=\rho_{\leq k}^{\prime}$ for some $k$, we mimic the strategy at $c(h)$ : $\sigma_{\text {Agt }}^{\prime}(h)=\sigma_{\text {Agt }}\left(\rho_{c(h)}^{\prime}\right)$. This way, $\rho^{\prime}$ is the outcome of $\sigma_{\text {Agt }}^{\prime}$ from $s$.

- otherwise we take the longest prefix $h_{\leq k}$ that is a prefix of $\rho^{\prime}$, and define $\sigma_{\text {Agt }}^{\prime}(h)=$ $\sigma_{\text {Agt }}\left(\rho_{c\left(h_{\leq k}\right)}^{\prime} \cdot h_{\geq k+1}\right)$.

We now show that $\sigma_{\text {Agt }}^{\prime}$ is a Nash equilibrium. Assume that one of the players changes her strategy while playing according to $\sigma_{\text {Agt }}^{\prime}$ : either the resulting outcome does not deviate from $\pi \cdot \tau^{\omega}$, in which case the payoff of that player is not improved; or it deviates at some point, and from that point on, $\sigma_{\text {Agt }}^{\prime}$ follows the same strategies as in $\sigma_{\text {Agt }}$. Assume that the resulting outcome is an improvement over $\rho^{\prime}$ for the player who deviated. The suffix of the play after the deviation is the suffix of a play of $\sigma_{\text {Agt }}$ after a deviation by the same player. By construction, both plays have the same sets of visited and infinitely-visited states. Hence we have found an advantageous deviation from $\sigma_{\mathrm{Agt}}$ for one player, contradicting the fact that $\sigma_{\text {Agt }}$ is a Nash equilibrium.

3.2. Encoding the value problem as a constrained NE existence problem. We now give a reduction that will be used to infer hardness results for the constrained NE existence problem from the hardness of the value problem (as defined in Section 2.4): this will be the case when the hardness proof for the value problem involves the construction of a game satisfying the hypotheses of the proposition.

Proposition 3.2. Let $\mathcal{G}=\left\langle\right.$ States, Agt, Act, Mov, Tab, $\left.\left(\precsim_{A}\right)_{A \in \mathrm{Agt}}\right\rangle$ be a two-player zerosum game played between players $A$ and $B$, such that:

- the preference relation $\precsim_{A}$ for player $A$ is total, Noetherian and almost-well-founded (see Section 2.1);

- $\mathcal{G}$ is determined, i.e., for all play $\pi$ :

$$
\left[\exists \sigma_{A} \cdot \forall \sigma_{B} \cdot \pi \precsim{ }_{A} \operatorname{Out}\left(\sigma_{A}, \sigma_{B}\right)\right] \quad \Leftrightarrow \quad\left[\forall \sigma_{B} \cdot \exists \sigma_{A} \cdot \pi \precsim_{A} \operatorname{Out}\left(\sigma_{A}, \sigma_{B}\right)\right] .
$$


Let $\mathcal{G}^{\prime}$ be the (non-zero-sum) game obtained from $\mathcal{G}$ by replacing the preference relation of player $B$ by the one where all plays are equivalent. Then, for every state s, for every play $\pi$ from $s$, the two following properties are equivalent:

(i) there is a Nash equilibrium in $\mathcal{G}^{\prime}$ from $s$ with outcome $\rho$ such that $\pi \mathscr{L}_{A} \rho$;

(ii) player $A$ cannot ensure $\pi$ from $s$ in $\mathcal{G}$.

Proof. In this proof, $\sigma_{A}$ and $\sigma_{A}^{\prime}$ (resp. $\sigma_{B}$ and $\sigma_{B}^{\prime}$ ) refer to player- $A$ (resp. player- $B$ ) strategies. Furthermore we will write $\operatorname{Out}\left(\sigma_{A}, \sigma_{B}\right)$ instead of $\operatorname{Out}_{\mathcal{G}}\left(s,\left(\sigma_{A}, \sigma_{B}\right)\right)$.

We first assume there is a Nash equilibrium $\left(\sigma_{A}, \sigma_{B}\right)$ in $\mathcal{G}^{\prime}$ from $s$ such that $\pi \mathcal{L}_{A}$ $\operatorname{Out}\left(\sigma_{A}, \sigma_{B}\right)$. Since $\precsim_{A}$ is total, Out $\left(\sigma_{A}, \sigma_{B}\right) \prec_{A} \pi$. Consider a strategy $\sigma_{A}^{\prime}$ of player $A$ in $\mathcal{G}$. As $\left(\sigma_{A}, \sigma_{B}\right)$ is a Nash equilibrium, it holds that $\operatorname{Out}\left(\sigma_{A}^{\prime}, \sigma_{B}\right) \precsim_{A} \operatorname{Out}\left(\sigma_{A}, \sigma_{B}\right)$, which implies $\operatorname{Out}\left(\sigma_{A}^{\prime}, \sigma_{B}\right) \prec_{A} \pi$. We conclude that condition (ii) holds.

Assume now property $(i i)$. As the preference relation is Noetherian, we can select $\pi^{+}$which is the largest element for $\precsim A$ which can be ensured by player $A$. Let $\sigma_{A}$ be a corresponding strategy: for every strategy $\sigma_{B}, \pi^{+} \precsim_{A} \operatorname{Out}\left(\sigma_{A}, \sigma_{B}\right)$. Towards a contradiction, assume now that for every strategy $\sigma_{B}^{\prime}$, there exists a strategy $\sigma_{A}^{\prime}$ such that $\pi^{+} \prec_{A} \operatorname{Out}\left(\sigma_{A}^{\prime}, \sigma_{B}^{\prime}\right)$. Consider the set $S$ of such outcomes, and define $\pi^{\prime}$ as its minimal element (this is possible since the order $\precsim_{A}$ is almost-well-founded). Notice then that $\pi^{+} \prec_{A} \pi^{\prime}$, and also that for every strategy $\sigma_{B}^{\prime}$, there exists a strategy $\sigma_{A}^{\prime}$ such that $\pi^{\prime} \precsim_{A} \operatorname{Out}\left(\sigma_{A}^{\prime}, \sigma_{B}^{\prime}\right)$. Then, as the game is determined, we get that there exists some strategy $\sigma_{A}^{\prime}$ such that for all strategy $\sigma_{B}^{\prime}$, it holds that $\pi^{\prime} \precsim_{A} \operatorname{Out}\left(\sigma_{A}^{\prime}, \sigma_{B}^{\prime}\right)$. In particular, strategy $\sigma_{A}^{\prime}$ ensures $\pi^{\prime}$, which contradicts the maximality of $\pi^{+}$. Therefore, there is some strategy $\sigma_{B}^{\prime}$ for which for every strategy $\sigma_{A}^{\prime}, \pi^{+} \nprec_{A} \operatorname{Out}\left(\sigma_{A}^{\prime}, \sigma_{B}^{\prime}\right)$, which means $\operatorname{Out}\left(\sigma_{A}^{\prime}, \sigma_{B}^{\prime}\right) \precsim_{A} \pi^{+}$. We show now that $\left(\sigma_{A}, \sigma_{B}^{\prime}\right)$ is a witness for property $(i)$. We have seen on the one hand that $\pi^{+} \precsim A \operatorname{Out}\left(\sigma_{A}, \sigma_{B}^{\prime}\right)$, and on the other hand that $\operatorname{Out}\left(\sigma_{A}, \sigma_{B}^{\prime}\right) \precsim_{A} \pi^{+}$. By hypothesis, $\pi^{+} \prec_{A} \pi$, which yields $\operatorname{Out}\left(\sigma_{A}, \sigma_{B}^{\prime}\right) \prec_{A} \pi$. Pick another strategy $\sigma_{A}^{\prime}$ for player $A$. We have seen that $\operatorname{Out}\left(\sigma_{A}^{\prime}, \sigma_{B}^{\prime}\right) \precsim_{A} \pi^{+}$, which implies $\operatorname{Out}\left(\sigma_{A}^{\prime}, \sigma_{B}^{\prime}\right) \precsim_{A} \operatorname{Out}\left(\sigma_{A}, \sigma_{B}^{\prime}\right)$. This concludes the proof of $(i)$.

Remark 3.3. Any finite total preorder is obviously Noetherian and almost-well-founded. Also, any total preorder isomorphic to the set of non-positive integers is Noetherian and almost-well-founded. On the other hand, a total preorder isomorphic to $\left\{1 / n \mid n \in \mathbb{N}^{+}\right\}$is Noetherian but not almost-well-founded.

3.3. Encoding the value problem as a NE existence problem. We prove a similar result for the NE existence problem. In this reduction however, we have to modify the game by introducing a truly concurrent move at the beginning of the game. This is necessary since for turn-based games with $\omega$-regular winning conditions, there always exists a Nash equilibrium [15], hence the NE existence problem would be trivial.

Let $\mathcal{G}=\left\langle\right.$ States, Agt, Act, Mov, Tab, $\left.\left(\precsim_{A}\right)_{A \in \text { Agt }}\right\rangle$ be a two-player zero-sum game, with players $A$ and $B$. Given a state $s$ of $\mathcal{G}$ and a play $\pi$ from $s$, we define a game $\mathcal{G}_{\pi}$ by adding two states $s_{0}$ and $s_{1}$, in the very same way as in Figure 9, on page 16. From $s_{0}$, $A$ and $B$ play a matching-penny game to either go to the sink state $s_{1}$, or to the state $s$ in the game $\mathcal{G}$. We assume the same hypotheses than in Proposition 3.2 for the preference relation $\precsim_{A}$. Let $\pi^{+}$be in the highest equivalence class for $\precsim_{A}$ smaller than $\pi$ (it exists since $\precsim_{A}$ is Noetherian). In $\mathcal{G}_{\pi}$, player $B$ prefers runs that end in $s_{1}$ : formally, the preference relation $\precsim_{B}^{\pi}$ of player $B$ in $\mathcal{G}_{\pi}$ is given by $\pi^{\prime} \precsim_{B}^{\pi} \pi^{\prime \prime} \Leftrightarrow \pi^{\prime \prime}=s_{0} \cdot s_{1}^{\omega} \vee \pi^{\prime} \neq s_{0} \cdot s_{1}^{\omega}$. On the 
other hand, player $A$ prefers a path of $\mathcal{G}$ over going to $s_{1}$, if and only if, it is at least as good as $\pi$ : formally, the preference relation $\precsim_{A}^{\pi}$ for player $A$ in $\mathcal{G}_{\pi}$ is given by $s_{0} \cdot \pi^{\prime} \precsim{ }_{A}$ $s_{0} \cdot \pi^{\prime \prime} \Leftrightarrow \pi^{\prime} \precsim_{A} \pi^{\prime \prime}$, and $s_{0} \cdot s_{1}^{\omega} \sim_{A}^{\prime \prime} s_{0} \cdot \pi^{+}$.

Proposition 3.4. Let $\mathcal{G}=\left\langle\right.$ States, Agt, Act, Mov, Tab, $\left.\left(\precsim_{A}\right)_{A \in \mathrm{Agt}}\right\rangle$ be a two-player zerosum game, with players $A$ and $B$, such that:

- the preference relation $\precsim_{A}$ for player $A$ is total, Noetherian and almost-well-founded;

- $\mathcal{G}$ is determined.

Let $s$ be a state and $\pi$ be a play in $\mathcal{G}$ from s. Consider the game $\mathcal{G}_{\pi}$ defined above. Then the following two properties are equivalent:

(i) there is a Nash equilibrium in $\mathcal{G}_{\pi}$ from $s_{0}$;

(ii) player $A$ cannot ensure $\pi$ from $s$ in $\mathcal{G}$.

In particular, in a given class of games, if the hardness proof of the value problem involves a game which satisfies the hypotheses of the proposition, and if $\mathcal{G}_{\pi}$ belongs to that class, then the NE existence problem is at least as hard as the complement of the value problem. Proof. Assume that player $A$ cannot ensure at least $\pi$ from $s$ in $\mathcal{G}$, then according to Proposition 3.2, there is a Nash equilibrium $\left(\sigma_{A}, \sigma_{B}\right)$ in the game $\mathcal{G}^{\prime}$ of Proposition 3.2 with outcome $\rho$ such that $\pi \mathscr{L}_{A} \rho$. Consider the strategy profile $\left(\sigma_{A}^{\pi}, \sigma_{B}^{\pi}\right)$ in $\mathcal{G}_{\pi}$ that consists in playing the same action for both players in $s_{0}$, and then if the path goes to $s$, to play according to $\left(\sigma_{A}, \sigma_{B}\right)$. Player $B$ gets her best possible payoff under that strategy profile. If $A$ could change her strategy to get a payoff better than $s_{0} \cdot \pi^{+}$, then it would induce a strategy in $\mathcal{G}^{\prime}$ giving her a payoff better than $\rho$ (when played with strategy $\sigma_{B}$ ), which contradicts the fact that $\left(\sigma_{A}, \sigma_{B}\right)$ is a Nash equilibrium in $\mathcal{G}^{\prime}$. Therefore, $\left(\sigma_{A}^{\pi}, \sigma_{B}^{\pi}\right)$ is a Nash equilibrium in $\mathcal{G}_{\pi}$.

Conversely, assume that $A$ can ensure $\pi$ from $s$ in $\mathcal{G}$, and assume towards a contradiction that there is a Nash equilibrium $\left(\sigma_{A}^{\pi}, \sigma_{B}^{\pi}\right)$ in $\mathcal{G}_{\pi}$ from $s_{0}$. Then $\operatorname{Out}_{\mathcal{G}_{\pi}}\left(\sigma_{A}^{\pi}, \sigma_{B}^{\pi}\right)$ does not end in $s_{1}$, otherwise player $A$ could improve by switching to $s$ and then playing according to a strategy which ensures $\pi$. Also, $\operatorname{Out}_{\mathcal{G}_{\pi}}\left(\sigma_{A}^{\pi}, \sigma_{B}^{\pi}\right)$ cannot end in $\mathcal{G}$ either, otherwise player $B$ would improve by switching to $s_{1}$. We get that there is no Nash equilibrium in $\mathcal{G}_{\pi}$ from $s_{0}$, which concludes the proof.

\subsection{Encoding the constrained NE existence problem as an NE existence prob-} lem. The next proposition makes a link between the existence of a Nash equilibrium where a player gets a payoff larger than some bound and the (unconstrained) existence of a Nash equilibrium in a new game. This will allow, in some specific cases, to infer hardness results from the constrained NE existence problem to the NE existence problem.

The construction is inspired by the previous one, but it applies to a game with at least two players, and it applies to any two selected players as follows. Let $\mathcal{G}=\langle$ States, Agt, Act, Mov, Tab, $\left.\left(\precsim_{A}\right)_{A \in \text { Agt }}\right\rangle$ be a concurrent game, $s$ be a state of $\mathcal{G}, \rho$ be a play from $s$, and $A_{i}$ and $A_{j}$ be two distinct players. We define the new game $E\left(\mathcal{G}, A_{i}, A_{j}, \rho\right)$ again in the same way as on Figure 9. Now, in $s_{0}$, the two players $A_{i}$ and $A_{j}$ play a matching-penny game to either go to the sink state $s_{1}$, or to state $s$ in game $\mathcal{G}$.

For player $A_{j}$, the preference relation in $E\left(\mathcal{G}, A_{i}, A_{j}, \rho\right)$ is given by $\precsim_{A_{j}}^{\prime}$ such that $s_{0} \cdot s_{1}^{\omega} \prec_{A_{j}}^{\prime} s_{0} \cdot \pi$ and $s_{0} \cdot \pi \precsim_{A_{j}}^{\prime} s_{0} \cdot \pi^{\prime} \Leftrightarrow \pi \precsim_{A_{j}} \pi^{\prime}$, for any path $\pi$ and $\pi^{\prime}$ from $s$ in $\mathcal{G}$. For player $A_{i}$ the preference relation is $s_{0} \cdot \pi \precsim_{A_{i}}^{\prime} s_{0} \cdot \pi^{\prime} \Leftrightarrow \pi \precsim A_{i} \pi^{\prime}$, for any path $\pi$ and $\pi^{\prime}$ from $s$ in $\mathcal{G}$, and $s_{0} \cdot s_{1}^{\omega} \sim_{A_{i}} s_{0} \cdot \rho$. For any other player $A_{k}$, the preference relation 
$E\left(\mathcal{G}, A_{i}, A_{j}, \rho\right)$ is given by $s_{0} \cdot \pi \precsim_{A_{k}}^{\prime} s_{0} \cdot \pi^{\prime} \Leftrightarrow \pi \precsim A_{k} \pi^{\prime}$ for any path $\pi$ and $\pi^{\prime}$ from $s$ in $\mathcal{G}$, and $s_{0} \cdot s_{1}^{\omega} \sim_{A_{k}} s_{0} \cdot \rho$.

Proposition 3.5. Let $\mathcal{G}=\left\langle\right.$ States, Agt, Act, Mov, Tab, $\left.\left(\precsim_{A}\right)_{A \in \mathrm{Agt}}\right\rangle$ be a concurrent game, let $s$ be a state of $\mathcal{G}$, and $A_{i}$ and $A_{j}$ be two distinct players participating to $\mathcal{G}$. Pick two plays $\pi$ and $\rho$ from s such that $\rho \precsim_{A_{i}} \pi$. If there is a Nash equilibrium in $\mathcal{G}$ whose outcome is $\pi$, then there is a Nash equilibrium in $E\left(\mathcal{G}, A_{i}, A_{j}, \rho\right)$ whose outcome is $s_{0} \cdot \pi$. Reciprocally, if there is a Nash equilibrium in $E\left(\mathcal{G}, A_{i}, A_{j}, \rho\right)$ whose outcome is $s_{0} \cdot \pi$, then there is a Nash equilibrium in $\mathcal{G}$ whose outcome is $\pi$.

Proof. Assume that there is a Nash equilibrium $\sigma_{\text {Agt }}$ in $\mathcal{G}$ with outcome $\pi$ such that $\rho \precsim A_{i} \pi$. Then $s_{0} \cdot s_{1}^{\omega} \precsim_{A_{i}}^{\prime} s_{0} \cdot \pi$. Consider the strategy profile in $E\left(\mathcal{G}, A_{i}, A_{j}, \rho\right)$ that consists for $A_{i}$ and $A_{j}$ in playing different actions in $s_{0}$ and when the path goes to $s$, to play according to $\sigma_{\text {Agt }}$. Players $A_{i}$ and $A_{j}$ have no interest in changing their strategies in $s_{0}$, since for $A_{j}$ all plays of $\mathcal{G}$ are better than $s_{0} \cdot s_{1}^{\omega}$, and for $A_{i}$ the play $s_{0} \cdot \pi$ is better than $s_{0} \cdot s_{1}^{\omega}$. Hence, this is a Nash equilibrium in game $E\left(\mathcal{G}, A_{i}, A_{j}, \rho\right)$.

Reciprocally, if there is a Nash equilibrium in $E\left(\mathcal{G}, A_{i}, A_{j}, \rho\right)$, its outcome cannot end in $s_{1}$, since $A_{j}$ would have an interest in changing her strategy in $s_{0}$ (all plays of $\mathcal{G}$ are then better for her). The strategies followed from $s$ thus defines a Nash equilibrium in $\mathcal{G}$.

If we consider a class of games such that $E\left(\mathcal{G}, A_{i}, A_{j}, \rho\right)$ belongs to that class when $\mathcal{G}$ does, then the NE existence problem is then at least as hard as the constrained NE existence problem. Note however that the reduction assumes lower bounds on the payoffs, and we do not have a similar result for upper bounds on the payoffs. For instance, as we will see in Section 6, for a conjunction of Büchi objectives, we do not know whether the NE existence problem is in $\mathrm{P}$ (as the value problem) or NP-hard (as is the existence of an equilibrium where all the players are losing).

\section{The Suspect Game}

In this section, we construct an abstraction of a multi-player game $\mathcal{G}$ as a two-player zerosum game $\mathcal{H}$, such that there is a correspondence between Nash equilibria in $\mathcal{G}$ and winning strategies in $\mathcal{H}$ (formalised in forthcoming Theorem 4.5). This transformation does not require the game to be finite and is conceptually much deeper than the reductions given in the previous section; it will allow us to use algorithmic techniques from zero-sum games to compute Nash equilibria and hence solve the value and (constrained) NE existence problems in various settings.

4.1. Construction of the suspect game. We fix a concurrent game $\mathcal{G}=\langle$ States, Agt, Act, Mov, Tab, $\left.\left(\precsim_{A}\right)_{A \in \mathrm{Agt}}\right\rangle$ for the rest of the section, and begin with introducing a few extra definitions.

Definition 4.1. A strategy profile $\sigma_{\mathrm{Agt}}$ is a trigger profile for a play $\pi$ from some state $s$ if, for every player $A \in \mathrm{Agt}$, for every strategy $\sigma_{A}^{\prime}$ of player $A$, the path $\pi$ is at least as good as the outcome of $\sigma_{\mathrm{Agt}}\left[A \mapsto \sigma_{A}^{\prime}\right]$ from $s$ (that is, $\operatorname{Out}\left(s, \sigma_{\mathrm{Agt}}\left[A \mapsto \sigma_{A}^{\prime}\right]\right) \precsim_{A} \pi$ ).

The following result is folklore and a direct consequence of the definition:

Lemma 4.2. A Nash equilibrium is a trigger profile for its outcome. Reciprocally, a strategy profile which is trigger profile for its outcome is a Nash equilibrium. 
Definition 4.3 ([4]). Given two states $s$ and $s^{\prime}$, and a move $m_{\mathrm{Agt}}$, the set of suspect players for $\left(s, s^{\prime}\right)$ and $m_{\text {Agt }}$ is the set

$$
\operatorname{Susp}\left(\left(s, s^{\prime}\right), m_{\mathrm{Agt}}\right)=\left\{A \in \operatorname{Agt} \mid \exists m^{\prime} \in \operatorname{Mov}(s, A) . \operatorname{Tab}\left(s, m_{\mathrm{Agt}}\left[A \mapsto m^{\prime}\right]\right)=s^{\prime}\right\} .
$$

Given a path $\rho$ and a strategy profile $\sigma_{\mathrm{Agt}}$, the set of suspect players for $\rho$ and $\sigma_{\mathrm{Agt}}$ is the set of players that are suspect along each transition of $\rho$, i.e., it is the set

$$
\operatorname{Susp}\left(\rho, \sigma_{\text {Agt }}\right)=\left\{A \in \operatorname{Agt}|\forall i<| \rho \mid . A \in \operatorname{Susp}\left(\left(\rho_{=i}, \rho_{=i+1}\right), \sigma_{\text {Agt }}\left(\rho_{\leq i}\right)\right)\right\} .
$$

Intuitively, player $A \in \operatorname{Agt}$ is a suspect for transition $\left(s, s^{\prime}\right)$ and move $m_{\text {Agt }}$ if she can unilaterally change her action to activate the transition $\left(s, s^{\prime}\right):$ if $s^{\prime} \neq \operatorname{Tab}\left(s, m_{\text {Agt }}\right)$, then this may be due to a deviation from $m_{\text {Agt }}$ of any of the players in the set $\operatorname{Susp}\left(\left(s, s^{\prime}\right), m_{\text {Agt }}\right)$, and no one else. If $s^{\prime}=\operatorname{Tab}\left(s, m_{\mathrm{Agt}}\right)$, it may simply be the case that no one has deviated, so everyone is a potential suspect for the next moves. Similarly, we easily infer that player $A$ is in $\operatorname{Susp}\left(\rho, \sigma_{\mathrm{Agt}}\right)$ if, and only if, there is a strategy $\sigma_{A}^{\prime} \operatorname{such}$ that $\operatorname{Out}\left(s, \sigma_{\mathrm{Agt}}\left[A \mapsto \sigma_{A}^{\prime}\right]\right)=\rho$.

Note that the notion of suspect players requires moves and arenas to be deterministic, and therefore everything which follows assumes the restriction to pure strategy profiles and to deterministic game structures.

We fix a play $\pi$ in $\mathcal{G}$. From game $\mathcal{G}$ and play $\pi$, we build the suspect game $\mathcal{H}(\mathcal{G}, \pi)$, which is a two-player turn-based game defined as follows. The players in $\mathcal{H}(\mathcal{G}, \pi)$ are named Eve and Adam. Since $\mathcal{H}(\mathcal{G}, \pi)$ is turn-based, its state space can be written as the disjoint union of the set $V_{\exists}$ controlled by Eve, which is (a subset of) States $\times 2^{\text {Agt }}$, and the set $V_{\forall}$ controlled by Adam, which is (a subset of) States $\times 2^{\text {Agt }} \times$ Act $^{\text {Agt }}$. The game is played in the following way: from a configuration $(s, P)$ in $V_{\exists}$, Eve chooses a legal move $m_{\text {Agt }}$ from $s$; the next state is $\left(s, P, m_{\text {Agt }}\right)$; then Adam chooses some state $s^{\prime}$ in States, and the new configuration is $\left(s^{\prime}, P \cap \operatorname{Susp}\left(\left(s, s^{\prime}\right), m_{\mathrm{Agt}}\right)\right)$. In particular, when the state $s^{\prime}$ chosen by Adam is such that $s^{\prime}=\operatorname{Tab}\left(s, m_{\text {Agt }}\right)$ (we say that Adam obeys Eve when this is the case), then the new configuration is $\left(s^{\prime}, P\right)$.

We define projections $p r o j_{1}$ and $p r o j_{2}$ from $V_{\exists}$ on States and $2^{\text {Agt }}$, resp., by $\operatorname{proj}_{1}(s, P)=$ $s$ and $\operatorname{proj}_{2}(s, P)=P$. We extend these projections to paths in a natural way (but only using Eve's states in order to avoid stuttering), letting $\operatorname{proj}_{1}\left(\left(s_{0}, P_{0}\right) \cdot\left(s_{0}, P_{0}, m_{0}\right) \cdot\left(s_{1}, P_{1}\right) \cdots\right)=$ $s_{0} \cdot s_{1} \cdots$. For any play $\rho, \operatorname{proj}_{2}(\rho)$ (seen as a sequence of sets of players of $\mathcal{G}$ ) is nonincreasing, therefore its limit $\lambda(\rho)$ is well defined. We notice that if $\lambda(\rho) \neq \varnothing$, then $\operatorname{proj}_{1}(\rho)$ is a play in $\mathcal{G}$. An outcome $\rho$ is winning for Eve, if for all $A \in \lambda(\rho)$, it holds $\operatorname{proj}_{1}(\rho) \precsim A \pi$. The winning region $W(\mathcal{G}, \pi)$ (later simply denoted by $W$ when $\mathcal{G}$ and $\pi$ are clear from the context) is the set of configurations of $\mathcal{H}(\mathcal{G}, \pi)$ from which Eve has a winning strategy. Intuitively Eve tries to have the players play a Nash equilibrium, and Adam tries to disprove that it is a Nash equilibrium, by finding a possible deviation that improves the payoff of one of the players.

4.2. Correctness of the suspect-game construction. The next lemma establishes a correspondence between winning strategies in $\mathcal{H}(\mathcal{G}, \pi)$ and trigger profiles (and therefore Nash equilibria) in $\mathcal{G}$.

Lemma 4.4. Let $s$ be a state of $\mathcal{G}$ and $\pi$ be a play from $s$ in $\mathcal{G}$. The following two conditions are equivalent: 
- Eve has a winning strategy in $\mathcal{H}(\mathcal{G}, \pi)$ from $(s, \mathrm{Agt})$, and its outcome $\rho^{\prime}$ from ( $\left.s, \mathrm{Agt}\right)$ when Adam obeys Eve is such that $\operatorname{proj}_{1}\left(\rho^{\prime}\right)=\rho$;

- there is a trigger profile for $\pi$ in $\mathcal{G}$ from state $s$ whose outcome from $s$ is $\rho$.

Proof. Assume there is a winning strategy $\sigma_{\exists}$ for Eve in $\mathcal{H}(\mathcal{G}, \pi)$ from $(s$, Agt), whose outcome from $\left(s\right.$, Agt) when Adam obeys Eve is $\rho^{\prime}$ with $\operatorname{proj}_{1}\left(\rho^{\prime}\right)=\rho$. We define the strategy profile $\sigma_{\text {Agt }}$ according to the actions played by Eve. Pick a history $g=s_{1} s_{2} \cdots s_{k+1}$, with $s_{1}=s$. Let $h$ be the outcome of $\sigma_{\exists}$ from $s$ ending in a state of $V_{\exists}$ and such that $\operatorname{proj}_{1}(h)=$ $s_{1} \cdots s_{k}$. This history is uniquely defined as follows: the first state of $h$ is ( $s_{1}$, Agt), and if its $(2 i+1)$-st state is $\left(s_{i}, P_{i}\right)$, then its $(2 i+2)$-nd state is $\left(s_{i}, P_{i}, \sigma_{\exists}\left(h_{\leq 2 i+1}\right)\right)$ and its $(2 i+3)$-rd state is $\left(s_{i+1}, P_{i} \cap \operatorname{Susp}\left(\left(s_{i}, s_{i+1}\right), \sigma_{\exists}\left(h_{\leq 2 i+1}\right)\right)\right)$. Now, write $\left(s_{k}, P_{k}\right)$ for the last state of $h$, and let $h^{\prime}=h \cdot\left(s_{k}, P_{k}, \sigma_{\exists}(h)\right) \cdot\left(s_{k+1}, P_{k} \cap \operatorname{Susp}\left(\left(s_{k}, s_{k+1}\right), \sigma_{\exists}(h)\right)\right)$. Then we define $\sigma_{\text {Agt }}(g)=\sigma_{\exists}\left(h^{\prime}\right)$. Notice that when $g \cdot s$ is a prefix of $\operatorname{proj}_{1}\left(\rho^{\prime}\right)$, then $g \cdot s \cdot \sigma_{\text {Agt }}(g \cdot s)$ is also a prefix of $\operatorname{proj}_{1}\left(\rho^{\prime}\right)$. In particular, $\operatorname{Out}\left(s, \sigma_{\mathrm{Agt}}\right)=\operatorname{proj}_{1}\left(\rho^{\prime}\right)=\rho$.

We now prove that $\sigma_{\text {Agt }}$ is a trigger profile for $\pi$. Pick a player $A \in$ Agt, a strategy $\sigma_{A}^{\prime}$ for player $A$, and let $g=\operatorname{Out}\left(s, \sigma_{\mathrm{Agt}}\left[A \mapsto \sigma_{A}^{\prime}\right]\right)$. With a play $g$, we associate a play $h$ in $\mathcal{H}(\mathcal{G}, \pi)$ in the same way as above. Then player $A$ is a suspect along all the transitions of $g$, so that she belongs to $\lambda(h)$. Now, as $\sigma_{\exists}$ is winning, $\operatorname{proj}_{1}(h) \precsim A \pi$, which proves that $\sigma_{\text {Agt }}$ is a trigger profile.

Conversely, assume that $\sigma_{\mathrm{Agt}}$ is a trigger profile for $\pi$ whose outcome is $\rho$, and define the strategy $\sigma_{\exists}$ by $\sigma_{\exists}(h)=\sigma_{\mathrm{Agt}}\left(\operatorname{proj}_{1}(h)\right)$. Notice that the outcome $\rho^{\prime}$ of $\sigma_{\exists}$ when Adam obeys Eve satisfies $\operatorname{proj}_{1}\left(\rho^{\prime}\right)=\rho$.

Let $\eta$ be an outcome of $\sigma_{\exists}$ from $s$, and $A \in \lambda(\eta)$. Then $A$ is a suspect for each transition along $\operatorname{proj}_{1}(\eta)$, which means that for all $i$, there is a move $m_{i}^{A}$ such that

$$
\operatorname{proj}_{1}\left(\eta_{=i+1}\right)=\operatorname{Tab}\left(\operatorname{proj}_{1}\left(\eta_{=i}\right), \sigma_{\mathrm{Agt}}\left(\operatorname{proj}_{1}\left(\eta_{\leq i}\right)\right)\left[A \mapsto m_{i}^{A}\right]\right) .
$$

Therefore there is a strategy $\sigma_{A}^{\prime}$ such that $\operatorname{proj}_{1}(\eta)=\operatorname{Out}\left(s, \sigma_{\mathrm{Agt}}\left[A \mapsto \sigma_{A}^{\prime}\right]\right)$. Since $\sigma_{\text {Agt }}$ is a trigger profile for $\pi$, it holds that $\operatorname{proj}_{1}(\eta) \precsim_{A} \pi$. As this holds for any $A \in \lambda(\eta), \sigma_{\exists}$ is winning.

We now state the correctness theorem for the suspect game construction.

Theorem 4.5. Let $\mathcal{G}=\left\langle\right.$ States, Agt, Act, Mov, Tab, $\left.\left(\precsim_{A}\right)_{A \in \mathrm{Agt}}\right\rangle$ be a concurrent game, $s$ be a state of $\mathcal{G}$, and $\pi$ be a play in $\mathcal{G}$. The following two conditions are equivalent:

- there is a Nash equilibrium $\sigma_{\text {Agt }}$ from $s$ in $\mathcal{G}$ whose outcome is $\pi$.

- there is a play $\rho$ from $(s, \mathrm{Agt})$ in $\mathcal{H}(\mathcal{G}, \pi)$,

(1) such that $\operatorname{proj}_{1}(\rho)=\pi$;

(2) along which Adam always obeys Eve; and

(3) such that for all indices $i$, there is a strategy $\sigma_{\exists}^{i}$ for Eve, for which any play in $\rho_{\leq i} \cdot \operatorname{Out}\left(\rho_{=i}, \sigma_{\exists}^{i}\right)$ is winning for Eve.

Proof. The Nash equilibrium is a trigger profile, and from Lemma 4.4, we get a winning strategy $\sigma_{\exists}$ in $\mathcal{H}(\mathcal{G}, \pi)$. The outcome $\rho$ of $\sigma_{\exists}$ from $s$ when Adam obeys Eve is such that $\pi=\operatorname{proj}_{1}(\rho)$ is the outcome of the Nash equilibrium. Now for all prefix $\rho_{\leq i}$, the strategy $\sigma_{\exists}^{i}: h \mapsto \sigma_{\exists}\left(\rho_{\leq i} \cdot h\right)$ is such that any play in $\rho_{\leq i} \cdot \operatorname{Out}\left(\rho_{=i}, \sigma_{\exists}^{i}\right)$ is winning for Eve.

Conversely, let $\rho^{\prime}$ be a path in $\mathcal{H}(\mathcal{G}, \pi)$ and assume it satisfies all three conditions. We define a strategy $\lambda_{\exists}$ that follows $\rho^{\prime}$ when Adam obeys. Along $\rho^{\prime}$, this strategy is defined as follows: $\lambda_{\exists}\left(\rho_{\leq 2 i}^{\prime}\right)=m_{\text {Agt }}$ such that $\operatorname{Tab}\left(\operatorname{proj}_{1}\left(\rho_{=i}^{\prime}\right), m_{\text {Agt }}\right)=\operatorname{proj}_{1}\left(\rho_{=i+1}^{\prime}\right)$. Such a legal 


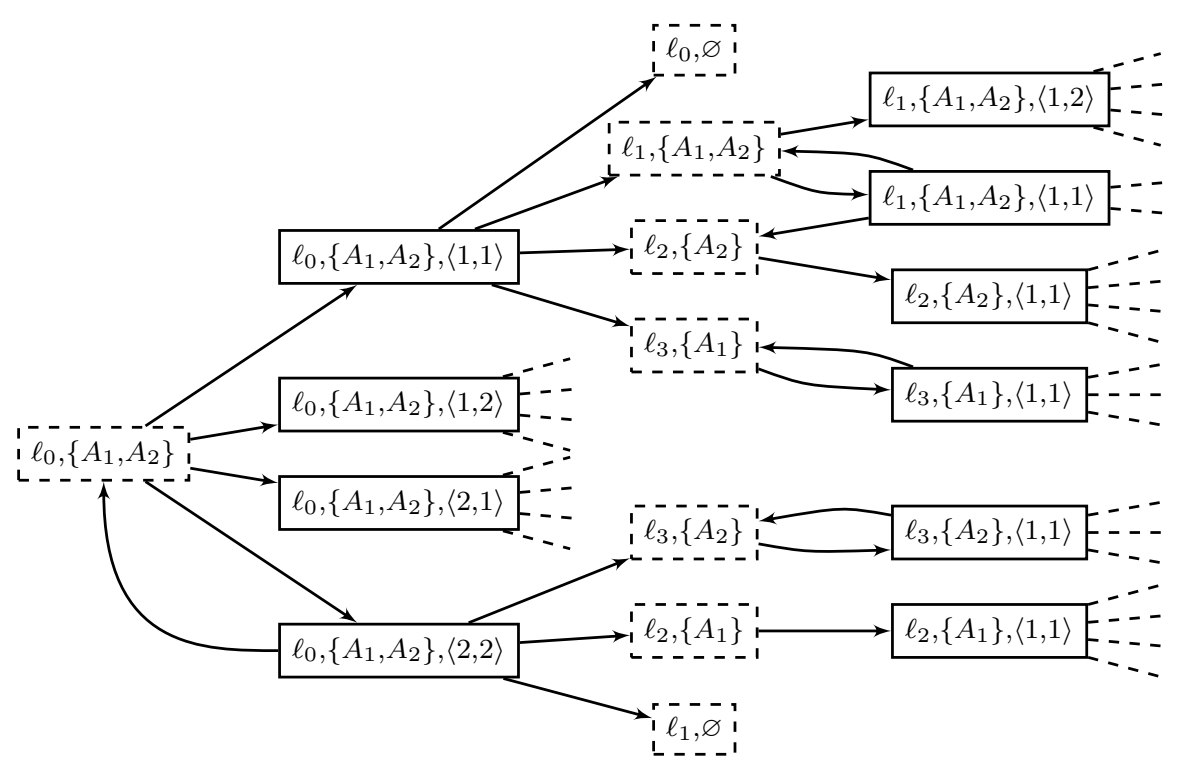

FiguRE 10. A small part of the suspect game for the game of Figure 3

move must exist since Adam obeys Eve along $\rho^{\prime}$ by condition 2. Now, if Adam deviates from the obeying strategy (at step $i$ ), we make $\lambda_{\exists}$ follow the strategy $\sigma_{\exists}^{i}$ (given by condition 3), which will ensure that the outcome is winning for Eve.

The outcomes of $\lambda_{\exists}$ are then either the path $\rho^{\prime}$, or a path $\rho^{\prime \prime}$ obtained by following a winning strategy after a prefix of $\rho^{\prime}$. The path $\rho^{\prime \prime}$ is losing for Adam, hence for all $A \in \lambda\left(\rho^{\prime}\right)$, $\rho^{\prime \prime} \precsim_{A} \rho^{\prime}$. This proves that $\lambda_{\exists}$ is a winning strategy. Applying Lemma 4.4, we obtain a strategy profile $\sigma_{\text {Agt }}$ in $\mathcal{G}$ that is a trigger profile for $\pi$. Moreover, the outcome of $\sigma_{\text {Agt }}$ from $s$ is $\operatorname{proj}_{1}\left(\rho^{\prime}\right)$ (using condition 1), so that $\sigma_{\text {Agt }}$ is a Nash equilibrium.

Remark 4.6. Assume the preference relations of each player $A$ in $\mathcal{G}$ are prefix-independent, i.e., for all plays $\rho$ and $\rho^{\prime}, \rho \precsim A \rho^{\prime}$ iff for all indices $i$ and $j, \rho_{\geq i} \precsim_{A} \rho_{\geq_{j}}^{\prime}$. Then the winning condition of Eve is also prefix-independent, and condition 3 just states that $\rho^{\prime}$ has to stay within the winning region of Eve. Note that, for prefix-dependent preference relations, condition 3 does not reduce to stay within the winning region of Eve: for instance, for safety objectives, if the losing states of all the players have been visited then any prolongation will satisfy the condition, even though it might leave the winning region of Eve.

Example 4.7. We depict on Figure 10 part of the suspect game for the game of Figure 3 . Note that the structure of $\mathcal{H}(\mathcal{G}, \pi)$ does not depend on $\pi$. Only the winning condition is affected by the choice of $\pi$.

In the rest of the paper, we use the suspect-game construction to algorithmically solve the NE existence problem and the constrained NE existence problem in finite games for large classes of preference relations. Before that we carefully analyse the size of the suspect game when the original game is finite.

4.3. Size of the suspect games when the original game is finite. We suppose that $\mathcal{G}$ is finite. At first sight, the number of states in $\mathcal{H}(\mathcal{G}, \pi)$ is exponential (in the number 
of players of $\mathcal{G})$. However, there are two cases for which we easily see that the number of states of $\mathcal{H}(\mathcal{G}, \pi)$ is actually only polynomial:

- if there is a state in which all the players have several possible moves, then the transition table (which is part of the input, as discussed in Remark 2.2) is also exponential in the number of players;

- if the game is turn-based, then the transition table is "small", but there is always at most one suspect player (unless all of them are suspects), so that the number of reachable states in $\mathcal{H}(\mathcal{G}, \pi)$ is also small.

We now prove that, due to the explicit encoding of the set of transitions (recall Remark 2.2, page 7), this can be generalised:

Proposition 4.8. Let $\mathcal{G}=\left\langle\right.$ States, Agt, Act, Mov, Tab, $\left.\left(\precsim_{A}\right)_{A \in \mathrm{Agt}}\right\rangle$ be a finite concurrent game and $\pi$ be a play in $\mathcal{G}$. The number of reachable configurations from States $\times\{$ Agt $\}$ in $\mathcal{H}(\mathcal{G}, \pi)$ is polynomial in the size of $\mathcal{G}$.

Proof. The game $\mathcal{H}(\mathcal{G}, \pi)$ contains the state $(s, \mathrm{Agt})$ and the states $\left(s, \mathrm{Agt}, m_{\mathrm{Agt}}\right)$, where $m_{\text {Agt }}$ is a legal move from $s$; the number of these states is bounded by $\mid$ States $|+|$ Tab $\mid$. The successors of those states that are not of the same form, are the $\left(t, \operatorname{Susp}\left((s, t), m_{\text {Agt }}\right)\right)$ with $t \neq \operatorname{Tab}\left(s, m_{\mathrm{Agt}}\right)$. If some player $A \in \operatorname{Agt}$ is a suspect for transition $(s, t)$, then besides $m_{A}$, she must have at least a second action $m^{\prime}$, for which $\operatorname{Tab}\left(s, m_{\text {Agt }}\left[A \mapsto m^{\prime}\right]\right)=t$. Thus the transition table from state $s$ has size at least $2^{\left|\operatorname{Susp}\left((s, t), m_{\mathrm{Agt}}\right)\right|}$. The successors of $\left(t, \operatorname{Susp}\left((s, t), m_{\mathrm{Agt}}\right)\right)$ are of the form $\left(t^{\prime}, P\right)$ or $\left(t^{\prime}, P, m_{\mathrm{Agt}}\right)$ where $P$ is a subset of $\operatorname{Susp}\left((s, t), m_{\mathrm{Agt}}\right)$; there can be no more than $(|\operatorname{States}|+|\mathrm{Tab}|) \cdot 2^{\left|\operatorname{Susp}\left((s, t), m_{\mathrm{Agt}}\right)\right|}$ of them, which is bounded by $(\mid$ States $|+| \mathrm{Tab} \mid) \cdot|\mathrm{Tab}|$. The total number of reachable states is then bounded by $(\mid$ States $|+|$ Tab $\mid) \cdot(1+(\mid$ States $|+|$ Tab $\mid) \cdot \mid$ Tab $\mid)$.

\section{Single-objective PReference Relations}

In this section we will be interested in finite games with single-objective preference relations.

The value problem for finite concurrent games with $\omega$-regular objectives has standard solutions in game theory; they are given in Table 1 (page 3). Let us briefly give some explanations. Most of the basic literature on two-player games focus on turn-based games, and in particular algorithms for solving two-player games with $\omega$-regular objectives only deal with turn-based games (see for instance [23, Chapter 2]). In particular, McNaughton developed an algorithm to solve turn-based parity games in time $O\left(\mid\right.$ States $\left.\left.|\cdot| \mathrm{Edg}\right|^{p-1}\right)$, where $p-1$ is the number of priorities [32]. Büchi games and co-Büchi games correspond to parity games with two priorities, hence they are solvable in polynomial time. Similarly reachability games and safety games can be transformed into Büchi games by making the target states absorbing. Hence turn-based game with these types of objectives can be solved in polynomial time.

Note however that we can reuse these algorithms in the concurrent case as follows. Any finite concurrent zero-sum game with objective $\Omega$ for player $A_{1}$ can be transformed into a turn-based zero-sum game with objective $\widetilde{\Omega}$ for player $A_{1}$ : the idea is to replace any edge labelled with pair of actions $\left\langle a_{1}, a_{2}\right\rangle$ into two consecutive transitions labelled with $a_{1}$ (belonging to player $A_{1}$ ) and with $a_{2}$ (belonging to player $A_{2}$ ). Furthermore $\Omega$ is an

$\omega$-regular condition, then so is $\widetilde{\Omega}$, and the type of the objective (reachability, Büchi, etc) is preserved (note however that this transformation only preserves Player $A_{1}$ objective). 
Hence the standard algorithm on the resulting turn-based game can be applied. Lower bounds for reachability/safety and Büchi/co-Büchi games are also folklore results, and can be obtained by encoding the circuit-value problem (we recall the encoding in Section 5.3.3).

We now focus on the NE existence problem and on the constrained NE existence problem when each player has a single ( $\omega$-regular) objective using the suspect game construction. The results are summarised in the second column of Table 1.

Streett and Muller objectives are not explicitly mentioned in the rest of the section. The complexity of their respective (constrained) NE existence problems, which is given in Table1, can easily be inferred from other ones. The $\mathrm{P}_{\|}^{\mathrm{NP}}$-hardness for the NE existence problem with Streett objectives follows from the corresponding hardness for parity objectives (parity objectives can be encoded efficiently as Streett objectives). Hardness for the NE existence problem in Muller games, is deduced from hardness of the value problem (which holds for turn-based games), applying Proposition 3.4. For both objectives, membership in PSPACE follows from PSPACE membership for objectives given as Boolean circuits, since they can efficiently be encoded as Boolean circuits.

We fix for the rest of the section a multi-player finite game $\mathcal{G}=\langle$ States, Agt, Act, Mov, Tab, $\left.\left(\precsim_{A}\right)_{A \in \text { Agt }}\right\rangle$, and we assume that each $\precsim_{A}$ is single-objective, given by set $\Omega_{A}$.

Remark 5.1. Let us come back to Remark 2.2 on our choice of an explicit encoding for the set of transitions. Assuming more compact encodings, the complexity of computing Nash equilibria for qualitative objectives does not allow to distinguish between the intrinsic complexity of the objectives. Indeed, in the formalism of [30, the transition function is given in each state by a finite sequence $\left(\left(\phi_{0}, s_{0}\right), \ldots,\left(\phi_{h}, s_{h}\right)\right)$, where $s_{i} \in$ States, and $\phi_{i}$ is a boolean combination of propositions $(A=m)$ that evaluates to true iff agent $A$ chooses action $m$. The transition table is then defined as follows: Tab $\left(s, m_{\text {Agt }}\right)=s_{j}$ iff $j$ is the smallest index such that $\phi_{j}$ evaluates to true when, for every player $A \in \mathrm{Agt}, A$ chooses action $m_{A}$. It is required that the last boolean formula $\phi_{h}$ be $T$, so that no agent can enforce a deadlock.

We can actually state the following result, whose proof is postponed to the Appendix on page 69.

Proposition 5.2. For finite concurrent games with compact encoding of transition functions and with reachability/Büchi/safety objectives, the constrained NE existence problems is PSPACE-hard.

Remark 5.3. It is first interesting to notice that given two plays $\pi$ and $\pi^{\prime}$ the suspect games $\mathcal{H}(\mathcal{G}, \pi)$ and $\mathcal{H}\left(\mathcal{G}, \pi^{\prime}\right)$ only differ in their winning conditions. In particular, the structure of the game only depends on $\mathcal{G}$, and has polynomial size (see Proposition 4.8). We denote it with $\mathcal{J}(\mathcal{G})$. Moreover, as each relation $\precsim A$ is given by a single objective $\Omega_{A}$, the winning condition for Eve in $\mathcal{H}(\mathcal{G}, \pi)$ rewrites as: for every $A \in \lambda(\rho) \cap \operatorname{Los}(\pi)$, $\operatorname{proj}_{1}(\rho)$ is losing (in $\mathcal{G}$ ) for player $A$, where $\operatorname{Los}(\pi)$ is the set of players losing along $\pi$ in $\mathcal{G}$. This winning condition only depends on $\operatorname{Los}(\pi)$ (not on the precise value of play $\pi$ ). Therefore in this section, the suspect game is denoted with $\mathcal{H}(\mathcal{G}, L)$, where $L \subseteq$ Agt, and Eve wins play $\rho$ if, for every $A \in \lambda(\rho) \cap L, A$ loses along $\operatorname{proj}_{1}(\rho)$ in $\mathcal{G}$. In many cases we will be able to simplify this winning condition, and to obtain simple algorithms to the corresponding problems.

We now distinguish between the winning objectives of the players. There are some similarities in some of the cases (for instance safety and co-Büchi objectives), but they nevertheless all require specific techniques and proofs. 
5.1. Reachability objectives. The value problem for a reachability winning condition is P-complete. Below, we design a non-deterministic algorithm that runs in polynomial time for solving the constrained NE existence problem. We then end this subsection with a NPhardness proof of the constrained NE existence problem and NE existence problem. In the end, we prove the following result:

Theorem 5.4. For finite concurrent games with single reachability objectives, the NE existence problem and the constrained NE existence problem are NP-complete.

5.1.1. Reduction to a safety game. We assume that for every player $A, \Omega_{A}$ is a single reachability objective given by target set $T_{A}$. Given $L \subseteq$ Agt, in the suspect game $\mathcal{H}(\mathcal{G}, L)$, we show that the objective of Eve reduces to a safety objective. We define the safety objective $\Omega_{L}$ in $\mathcal{H}(\mathcal{G}, L)$ by the set $T_{L}=\left\{(s, P) \mid \exists A \in P \cap L . s \in T_{A}\right\}$ of target states.

Lemma 5.5. Eve has a winning strategy in game $\mathcal{H}(\mathcal{G}, L)$ iff Eve has a winning strategy in game $\mathcal{J}(\mathcal{G})$ with safety objective $\Omega_{L}$.

Proof. We first show that any play in $\Omega_{L}$ is winning in $\mathcal{H}(\mathcal{G}, L)$. Let $\rho \in \Omega_{L}$, and let $A \in \lambda(\rho) \cap L$. Toward a contradiction assume that $\operatorname{Occ}\left(\operatorname{proj}_{1}(\rho)\right) \cap T_{A} \neq \varnothing$ : there is a state $(s, P)$ along $\rho$ with $s \in T_{A}$. Obviously $\lambda(\rho) \subseteq P$, which implies that $A \in P \cap L$. This contradicts the fact that $\rho \notin \Omega_{L}$. We have shown so far that any winning strategy for Eve in $\mathcal{J}(\mathcal{G})$ with safety objective $\Omega_{L}$ is a winning strategy for Eve in $\mathcal{H}(\mathcal{G}, L)$.

Now assume that Eve has no winning strategy in game $\mathcal{J}(\mathcal{G})$ with safety objective $\Omega_{L}$. Turn-based games with safety objectives being determined, Adam has a strategy $\sigma_{\forall}$ which ensures that no outcome of $\sigma_{\forall}$ is in $\Omega_{L}$. If $\rho \in \operatorname{Out}\left(\sigma_{\forall}\right)$, there is a state $(s, P)$ along $\rho$ such that there is $A \in P \cap L$ with $s \in T_{A}$. We now modify the strategy of Adam such that as soon as such a state is reached we switch from $\sigma_{\forall}$ to the strategy that always obeys Eve. This ensures that in every outcome $\rho^{\prime}$ of the new strategy, we reach a state $(s, P)$ such that there is $A \in P \cap L$ with $s \in T_{A}$, and $\lambda\left(\rho^{\prime}\right)=P$. This Adam's strategy thus makes Eve lose the game $\mathcal{H}(\mathcal{G}, L)$, and Eve has no winning strategy in game $\mathcal{H}(\mathcal{G}, L)$.

5.1.2. Algorithm. The algorithm for solving the constrained NE existence problem in a game where each player has a single reachability objective relies on Theorem 4.5 and Proposition 3.1, and on the above analysis:

(i) guess a lasso-shaped play $\rho=\tau_{1} \cdot \tau_{2}^{\omega}$ (with $\left|\tau_{i}\right| \leq 2 \mid$ States $\left.\right|^{2}$ ) in $\mathcal{J}(\mathcal{G})$, such that Adam obeys Eve along $\rho$, and $\pi=\operatorname{proj}_{1}(\rho)$ satisfies the constraint on the payoff;

(ii) compute the set $W(\mathcal{G}, \operatorname{Los}(\pi))$ of states that are winning for Eve in the suspect game $\mathcal{H}(\mathcal{G}, \operatorname{Los}(\pi))$, where $\operatorname{Los}(\pi)$ is the set of losing players along $\pi$;

(iii) check that $\rho$ stays in $W(\mathcal{G}, \operatorname{Los}(\pi))$.

First notice that this algorithm is non-deterministic and runs in polynomial time: the witness $\rho$ guessed in step (i) has size polynomial; the suspect game $\mathcal{H}(\mathcal{G}, \operatorname{Los}(\pi))$ has also polynomial size (Proposition 4.8); Step (ii) can be done in polynomial time using a standard attractor computation [23, Sect. 2.5.1] as the game under analysis is equivalent to a safety game (Lemma 5.5); finally step (iii) can obviously be performed in polynomial time.

Step (i) ensures that conditions 2 and 1 of Theorem 4.5 hold for $\rho$ and step (iii) ensures condition [3. Correctness of the algorithm then follows from Theorem 4.5] and Proposition 3.1 . 
5.1.3. Hardness. We prove NP-hardness of the constrained NE existence problem by encoding an instance of 3SAT as follows. We assume set of atomic propositions $\mathrm{AP}=\left\{x_{1}, \ldots, x_{k}\right\}$, and we let $\phi=\bigwedge_{i=1}^{n} c_{i}$ where $c_{i}=\ell_{i, 1} \vee \ell_{i, 2} \vee \ell_{i, 3}$ where $\ell_{i, j} \in\left\{x_{k}, \neg x_{k} \mid 1 \leq k \leq p\right\}$. We build the turn-based game $\mathcal{G}_{\phi}$ with $n+1$ players Agt $=\left\{A, C_{1}, \ldots, C_{n}\right\}$ as follows: for every $1 \leq k \leq p$, player $A$ chooses to visit either location $x_{k}$ or location $\neg x_{k}$. Location $x_{k}$ is winning for player $C_{i}$ if, and only if, $x_{k}$ is one of the literals in $c_{i}$, and similarly location $\neg x_{k}$ is winning for $C_{i}$ if, and only if, $\neg x_{k}$ is one of the literals of $c_{i}$. The construction is illustrated on Figure 11, with the reachability objectives defined as $\Omega_{C_{i}}=\left\{\ell_{i, 1}, \ell_{i, 2}, \ell_{i, 3}\right\}$ for $1 \leq i \leq n$. Now, it is easy to check that this game has a Nash equilibrium with payoff 1 for all players $\left(C_{i}\right)_{1 \leq i \leq n}$ if, and only if, $\phi$ is satisfiable.

We prove hardness for the NE existence problem by using the transformation described in Section 3.4 once for each player. We define the game $\mathcal{G}_{0}$ similar to $\mathcal{G}$ but with an extra player $C_{n+1}$ who does not control any state for now. For $1 \leq i \leq n$, we define $\mathcal{G}_{i}=E\left(\mathcal{G}_{i-1}, C_{i}, C_{n+1}, \rho\right)$, where $\rho$ is a winning path for $C_{i}$. The preference relation can be expressed in any $\mathcal{G}_{i}$ by a reachability condition, by giving to $C_{n+1}$ a target which is the initial state of $\mathcal{G}$. According to Proposition 3.5 there is a Nash equilibrium in $\mathcal{G}_{i}$ if, and only if, there is one in $\mathcal{G}_{i-1}$ where $C_{i}$ wins. Therefore there is a Nash equilibrium in $\mathcal{G}_{n}$ if, and only if, $\phi$ is satisfiable. This entails NP-hardness of the NE existence problem.

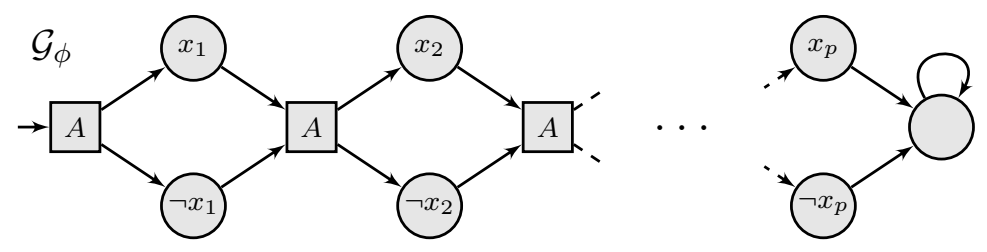

FiguRE 11. Reachability game for the reduction of 3SAT

5.2. Safety objectives. The value problem for safety objectives is P-complete. We next show that the constrained NE existence problem can be solved in NP, and conclude with NP-hardness of both the constrained NE existence problem and the NE existence problem. We hence prove:

Theorem 5.6. For finite games with single safety objectives, the NE existence problem and the constrained NE existence problem are NP-complete.

5.2.1. Reduction to a conjunction of reachability objectives. We assume $\Omega_{A}$ is a single safety objective given by set $T_{A}$. In the corresponding suspect game, we show that the goal of Eve is equivalent to a conjunction of reachability objectives. Let $L \subseteq$ Agt. In suspect game $\mathcal{H}(\mathcal{G}, L)$, we define several reachability objectives as follows: for each $A \in L$, we define $T_{A}^{\prime}=T_{A} \times\{P \mid P \subseteq$ Agt $\} \cup$ States $\times\{P \mid A \notin P\}$, and we write $\Omega_{A}^{\prime}$ for the corresponding reachability objectives.

Lemma 5.7. A play $\rho$ is winning for Eve in $\mathcal{H}(\mathcal{G}, L)$ iff $\rho \in \bigcap_{A \in L} \Omega_{A}^{\prime}$.

Proof. Let $\rho$ be a play in $\mathcal{H}(\mathcal{G}, L)$, and assume it is winning for Eve. Then, for each $A \in \lambda(\rho) \cap L, \rho \notin \Omega_{A}$, which means that the target set $T_{A}$ is visited along $\operatorname{proj}_{1}(\rho)$, and 
therefore $T_{A}^{\prime}$ is visited along $\rho$. If $A \notin \lambda(\rho)$, then a state $(s, P)$ with $A \notin P$ is visited by $\rho$ : the target set $T_{A}^{\prime}$ is visited. This implies that $\rho \in \bigcap_{A \in L} \Omega_{A}^{\prime}$.

Conversely let $\rho \in \bigcap_{A \in L} \Omega_{A}^{\prime}$. For every $A \in L, T_{A}^{\prime}$ is visited by $\rho$. Then, either $T_{A}$ is visited by $\operatorname{proj}_{1}(\rho)$ (which means that $\rho \notin \Omega_{A}$ ) or $A \notin \lambda(\rho)$. In particular, $\rho$ is a winning play for Eve in $\mathcal{H}(\mathcal{G}, L)$.

5.2.2. Algorithm for solving finite zero-sum turn-based games with a conjunction of reachability objectives. We now give a simple algorithm for solving zero-sum games with a conjunction of reachability objectives. This algorithm works in exponential time with respect to the size of the conjunction (we will see in Subsection 7.1.6 that the problem is PSPACEcomplete). However for computing Nash equilibria in safety games we will only use it for small (logarithmic size) conjunctions.

Let $\overline{\mathcal{G}}$ be a two-player turn-based game with a winning objective for Eve given as a conjunction of $k$ reachability objectives $\Omega_{1}, \ldots, \Omega_{k}$. We assume vertices of Eve and Adam in $\overline{\mathcal{G}}$ are $V_{\exists}$ and $V_{\forall}$ respectively, and that the initial vertex is $v_{0}$. The idea is to construct a new game $\overline{\mathcal{G}}^{\prime}$ that remembers the objectives that have been visited so far. The vertices of game $\overline{\mathcal{G}}^{\prime}$ controlled by Eve and Adam are $V_{\exists}^{\prime}=V_{\exists} \times 2^{\llbracket 1, k \rrbracket}$ and $V_{\forall}^{\prime}=V_{\forall} \times 2^{\llbracket 1, k \rrbracket}$ respectively. There is a transition from $(v, S)$ to $\left(v^{\prime}, S^{\prime}\right)$ iff there is a transition from $v$ to $v^{\prime}$ in the original game and $S^{\prime}=S \cup\left\{i \mid v^{\prime} \in \Omega_{i}\right\}$. The reachability objective $\Omega$ for Eve is given by target set States $\times \llbracket 1, k \rrbracket$. It is clear that there is a winning strategy in $\overline{\mathcal{G}}$ from $v_{0}$ for the conjunction of reachability objectives $\Omega_{1}, \ldots, \Omega_{k}$ iff there is a winning strategy in game $\overline{\mathcal{G}}^{\prime}$ from $\left(v_{0},\left\{i \mid v_{0} \in \Omega_{i}\right\}\right)$ for the reachability objective $\Omega$. The number of vertices of this new game is $\left|V_{\exists}^{\prime} \cup V_{\forall}^{\prime}\right|=\left|V_{\exists} \cup V_{\forall}\right| \cdot 2^{k}$, and the size of the new transition table $\mathrm{Tab}^{\prime}$ is bounded by $|\mathrm{Tab}| \cdot 2^{k}$, where Tab is the transition table of $\overline{\mathcal{G}}$. An attractor computation on $\overline{\mathcal{G}}^{\prime}$ is then done in time $\mathcal{O}\left(\left|V_{\exists}^{\prime} \cup V_{\forall}^{\prime}\right| \cdot\left|\mathrm{Tab}^{\prime}\right|\right)$, we obtain an algorithm for solving zero-sum games with a conjunction of reachability objectives, running in time $\mathcal{O}\left(2^{2 k} \cdot\left(\left|V_{\exists} \cup V_{\forall}\right| \cdot|\mathrm{Tab}|\right)\right)$.

5.2.3. Algorithm. The algorithm for solving the constrained NE existence problem for single reachability objectives could be copied and would then be correct. It would however not yield an NP upper bound. We therefore propose a refined algorithm:

(i) guess a lasso-shaped play $\rho=\tau_{1} \cdot \tau_{2}^{\omega}$ (with $\left|\tau_{i}\right| \leq \mid$ States $\left.\right|^{2}$ ) in $\mathcal{J}(\mathcal{G})$ such that Adam obeys Eve along $\rho$, and $\pi=\operatorname{proj}_{1}(\rho)$ satisfies the constraint on the payoff. Note that if $\operatorname{Los}(\pi)$ is the set of players losing in $\pi$, computing $W(\mathcal{G}, \operatorname{Los}(\pi))$ would require exponential time. We will avoid this expensive computation.

(ii) check that any Adam-deviation along $\rho$, say at position $i$ (for any $i$ ), leads to a state from which Eve has a strategy $\sigma_{\exists}^{i}$ to ensure that any play in $\rho_{\leq i} \cdot \operatorname{Out}\left(\sigma_{\exists}^{i}\right)$ is winning for her.

Step $(i i)$ can be done as follows: pick an Adam-state $\left(s\right.$, Agt, $\left.m_{\text {Agt }}\right)$ along $\rho$ and a successor $(t, P)$ such that $t \neq \operatorname{Tab}\left(s, m_{\text {Agt }}\right)$; we only need to show that $(t, P) \in W(\mathcal{G},(\operatorname{Los}(\pi) \backslash$ $\left.\left.\operatorname{Los}\left(\rho_{\leq i}\right)\right) \cap P\right)$. We can compute this set efficiently (in polynomial time) using the algorithm of the previous paragraph since $2^{|P|} \leq|\mathrm{Tab}|$ (using the same argument as in Proposition 4.8).

This non-deterministic algorithm, which runs in polynomial time, precisely implements Theorem 4.5, and therefore correctly decides the constrained NE existence problem. 


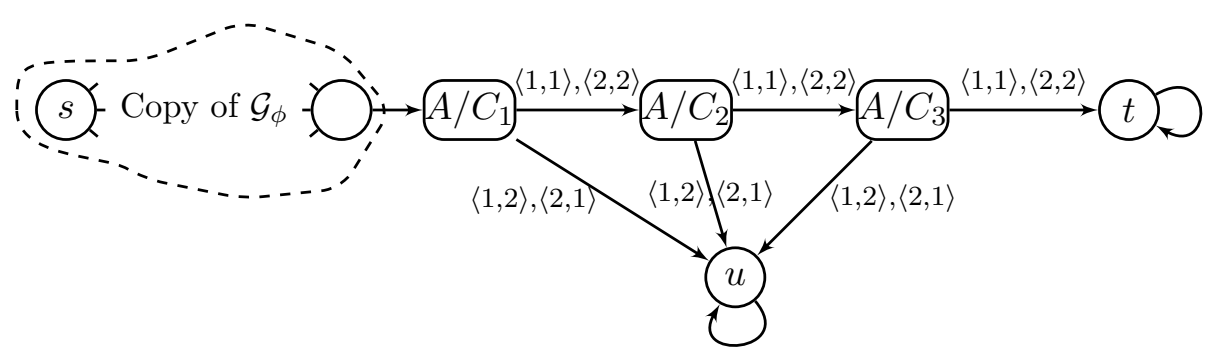

FigURE 12. Extending game $\mathcal{G}_{\phi}$ with final concurrent modules

5.2.4. Hardness. The NP-hardness for the constrained NE existence problem can be proven by encoding an instance of 3SAT using a game similar to that for reachability objectives, see Section 5.1. We only change the constraint which is now that all players $C_{i}$ should be losing, and we get the same equivalence.

The reduction of Lemma 3.4 cannot be used to deduce the hardness of the NE existence problem, since it assumes a lower bound on the payoff. Here the constraint is an upper bound ("each player should be losing"). We therefore provide an ad-hoc reduction in this special case, which is illustrated on Figure 12. We add some module at the end of the game to enforce that in an equilibrium, all players are losing. We add concurrent states between $A$ and each $C_{i}$ (named $A / C_{i}$ ). All players $C_{i}$ are trying to avoid $t$, and $A$ is trying to avoid $u$.

Since $A$ has no target in $\mathcal{G}_{\phi}$ she cannot lose before seeing $u$, and then she can always change her strategy in the concurrent states in order to go to $t$. Therefore an equilibrium always ends in $t$. A player $C_{i}$ whose target was not seen during game $\mathcal{G}_{\phi}$, can change her strategy in order to go to $u$ instead of $t$. That means that if there is an equilibrium, there was one in $\mathcal{G}_{\phi}$ where all $C_{i}$ are losing. Conversely, if there was such an equilibrium in $\mathcal{G}_{\phi}$, we can extend this strategy profile by one whose outcome goes to $t$ and it is an equilibrium in the new game. This concludes the NP-hardness of the NE existence problem.

5.3. Büchi objectives. The value problem for Büchi objectives is P-complete. In this subsection we design a polynomial-time algorithm for solving the constrained NE existence problem for Büchi objectives. The P-hardness of the NE existence problem can then be inferred from the P-hardness of the value problem, applying Propositions 3.2 and 3.4. Globally we prove the following result:

Theorem 5.8. For finite games with single Büchi objectives, the NE existence problem and the constrained $N E$ existence problem are P-complete.

5.3.1. Reduction to a co-Büchi game. We assume that for every player $A, \Omega_{A}$ is a Büchi objective given by target set $T_{A}$. Given $L \subseteq$ Agt, in the suspect game $\mathcal{H}(\mathcal{G}, L)$, we show that the objective of Eve is equivalent to a single co-Büchi objective. We define the co-Büchi objective $\Omega_{L}$ in $\mathcal{H}(\mathcal{G}, L)$ given by the target set $T_{L}=\left\{(s, P) \mid \exists A \in P \cap L\right.$. $\left.s \in T_{A}\right\}$. Notice that the target set is defined in the same way as for reachability objectives.

Lemma 5.9. A play $\rho$ is winning for Eve in $\mathcal{H}(\mathcal{G}, L)$ iff $\rho \in \Omega_{L}$. 
Proof. Assume that $\rho$ is winning for Eve in $\mathcal{H}(\mathcal{G}, L)$. Then for every $A \in \lambda(\rho) \cap L$, it holds $\operatorname{Inf}\left(\operatorname{proj}_{1}(\rho)\right) \cap T_{A}=\varnothing$. Toward a contradiction, assume that $\operatorname{Inf}(\rho) \cap T_{L} \neq \varnothing$. There exists $(s, P)$ such that there is $A \in P \cap L$ with $s \in T_{A}$, which appears infinitely often along $\rho$. In particular, $P=\lambda(\rho)$ (otherwise it would not appear infinitely often along $\rho$ ). Hence, we have found $A \in \lambda(\rho) \cap L$ such that $\operatorname{Inf}\left(\operatorname{proj}_{1}(\rho)\right) \cap T_{A} \neq \varnothing$, which is a contradiction. Therefore, $\rho \in \Omega_{L}$.

Assume $\rho \in \Omega_{L}$ : for every $(s, P)$ such that there exists $A \in P \cap L$ with $s \in T_{A},(s, P)$ appears finitely often along $\rho$. Let $A \in \lambda(\rho) \cap L$, and assume towards a contradiction that there is $s \in T_{A}$ such that $s$ appears infinitely often along $\operatorname{proj}_{1}(\rho)$. This means that $(s, \lambda(\rho))$ appears infinitely often along $\rho$, which contradicts the above condition. Therefore, $\rho$ is winning for Eve in $\mathcal{H}(\mathcal{G}, L)$.

5.3.2. Algorithm. As for reachability objectives, the winning region for Eve in $\mathcal{H}(\mathcal{G}, L)$ can be computed in polynomial time (since this is the winning region of a co-Büchi game, see Lemma 5.9 above). A non-deterministic algorithm running in polynomial time similar to the one for reachability objectives can therefore be inferred. However we can do better than guessing an appropriate lasso-shaped play $\rho$ by looking at the strongly connected components of the game: a strongly connected component of the game uniquely defines a payoff, which is that of all plays that visit infinitely often all the states of that strongly connected component. Using a clever partitioning of the set of strongly connected components of the game, we obtain a polynomial-time algorithm.

From now on and until the end of Subsection 5.3.2 we relax the hypotheses on the preference relations (that they are all single-objective with a Büchi condition). We present an algorithm in a more general context, since the same techniques will be used in Subsection 6.2.2 (and we chose to only present once the construction). For the rest of this subsection we therefore make the following assumptions on the preference relations $\left(\precsim_{A}\right)_{A \in \text { Agt }}$. For every player $A \in$ Agt:

(a) $\precsim A$ only depends on the set of states which is visited infinitely often: if $\rho$ and $\rho^{\prime}$ are two plays such that $\operatorname{Inf}(\rho)=\operatorname{Inf}\left(\rho^{\prime}\right)$ then $\rho \precsim_{A} \rho^{\prime}$ and $\rho^{\prime} \precsim_{A} \rho$;

(b) $\precsim_{A}$ is given by an ordered objective $\omega_{A}$ with preorder $\lesssim_{A}$, and $\lesssim_{A}$ is supposed to be monotonic;

(c) for every threshold $w^{A}$, we can compute in polynomial time $S^{A} \subseteq$ States such that $\operatorname{Inf}(\rho) \subseteq S^{A} \Leftrightarrow \rho \precsim A w^{A}$.

Obviously preferences given by single Büchi objectives do satisfy those hypotheses. At every place where it is relevant, we will explain how the particular case of single Büchi objectives is handled. Next we write $(\star)$ for the above assumptions, and $(\star)_{a}\left(\operatorname{resp} .(\star)_{b},(\star)_{c}\right)$ for only the first (resp. second, third) assumption.

We first characterise the 'good' plays in $\mathcal{J}(\mathcal{G})$ in terms of the strongly connected components they define: the strongly connected component defined by a play is the set of states that are visited infinitely often by the play. We fix for each player $A$, equivalence classes of plays $u^{A}$ and $w^{A}$, that represent lower- and upper-bounds for the constrained NE existence problem. Both can be represented as finite sets, representing the set of states which are visited infinitely often. For each $K \subseteq$ States, we write $v^{A}(K)$ for the equivalence class of all paths $\pi$ that visits infinitely often exactly $K$, i.e.: $\operatorname{Inf}(\pi)=K$. We also 
write $v(K)=\left(v^{A}(K)\right)_{A \in \text { Agt }}$. We look for a transition system $\langle K, E\rangle$, with $K \subseteq$ States and $E \subseteq K \times K$, for which the following properties hold:

(1) $u^{A} \lesssim_{A} v^{A}(K) \lesssim_{A} w^{A}$ for all $A \in \mathrm{Agt}$;

(2) $\langle K, E\rangle$ is strongly connected;

(3) $\forall k \in K$. $(k$, Agt $) \in W(\mathcal{G}, v(K))$;

(4) $\forall\left(k, k^{\prime}\right) \in E . \exists\left(k\right.$, Agt, $\left.m_{\text {Agt }}\right) \in W(\mathcal{G}, v(K)) \cdot \operatorname{Tab}\left(k, m_{\text {Agt }}\right)=k^{\prime}$;

(5) $(K \times\{\mathrm{Agt}\})$ is reachable from $(s, \mathrm{Agt})$ in $W(\mathcal{G}, v(K))$;

where $W(\mathcal{G}, v(K))$ is the winning region of Eve in suspect game $\mathcal{H}(\mathcal{G}, v(K)) 5$

If one can find one such transition system $\langle K, E\rangle$, then we will be able to build a lassoplay $\rho$ from $(s, \mathrm{Agt})$ in the suspect-game that will satisfy the conditions of Theorem 4.5 . Formally, we have the following lemma:

Lemma 5.10. Under hypothesis $(\star)_{a}$, there is a transition system $\langle K, E\rangle$ satisfying conditions 115 if, and only if, there is a path $\rho$ from $(s, \operatorname{Agt})$ in $\mathcal{H}(\mathcal{G}, v(K))$ that never gets out of $W(\mathcal{G}, v(K))$, along which Adam always obeys Eve, $u^{A} \lesssim_{A} v^{A}(K) \lesssim_{A} w^{A}$ for all $A \in \mathrm{Agt}$, and $\operatorname{proj}_{1}\left(\operatorname{Inf}(\rho) \cap V_{\exists}\right)=K$ (which implies that $\rho \in v^{A}(K)$ for all $\left.A\right)$.

Proof. The first implication is shown by building a path in $W(\mathcal{G}, v(K))$ that successively visits all the states in $K \times\{\mathrm{Agt}\}$ forever. Thanks to 5 , 2 and 4 (and the fact that Adam obeys Eve), such a path exists, and from 3 and 4 , this path remains in the winning region. From 1, we have the condition on the preferences. Conversely, consider such a path $\rho$, and let $K=\operatorname{proj}_{1}\left(\operatorname{Inf}(\rho) \cap V_{\exists}\right)$ and $E=\left\{\left(k, k^{\prime}\right) \in K^{2} \mid \exists\left(k, \operatorname{Agt}, m_{\text {Agt }}\right) \in \operatorname{Inf}(\rho) . \operatorname{Tab}\left(k, m_{\text {Agt }}\right)=k^{\prime}\right\}$. Condition 5 clearly holds. Conditions 1, 3 and 4 are easy consequences of the hypotheses and construction. We prove that $\langle K, E\rangle$ is strongly connected. First, since Adam obeys Eve and $\rho$ starts in $\left(k\right.$, Agt), we have $\lambda(\rho)=$ Agt. Now, take any two states $k$ and $k^{\prime}$ in $K$ : then $\rho$ visits $\left(k\right.$, Agt) and $\left(k^{\prime}\right.$, Agt) infinitely often, and there is a subpath of $\rho$ between those two states, all of which states appear infinitely often along $\rho$. Such a subpath gives rise to a path between $k$ and $k^{\prime}$, as required.

As a consequence, if $\langle K, E\rangle$ satisfies the five previous conditions, by Theorem 4.5, there is a Nash equilibrium whose outcome lies between the bounds $u^{A}$ and $w^{A}$. Our aim is to compute efficiently all maximal pairs $\langle K, E\rangle$ that satisfy the five conditions.

To that aim we define a recursive function SSG (standing for "solve sub-game"), working on transition systems, that will decompose efficiently any transition system that does not satisfy the five conditions above into polynomially many disjoint sub-transition systems via a decomposition into strongly connected components.

- if $K \times\{\mathrm{Agt}\} \subseteq W(\mathcal{G}, v(K))$, and if for all $\left(k, k^{\prime}\right) \in E$ there is a (k, Agt, $\left.m_{\mathrm{Agt}}\right)$ in $W(\mathcal{G}, v(K))$ s.t. $\operatorname{Tab}\left(k, m_{\mathrm{Agt}}\right)=k^{\prime}$, and finally if $\langle K, E\rangle$ is strongly connected, then we set SSG $(\langle K, E\rangle)=\{\langle K, E\rangle\}$. This means that conditions (2)-(4) are satisfied by $\langle K, E\rangle$.

- otherwise, we let

$$
\mathrm{SSG}(\langle K, E\rangle)=\bigcup_{\left\langle K^{\prime}, E^{\prime}\right\rangle \in \operatorname{SCC}(\langle K, E\rangle)} \mathrm{SSG}\left(T\left(\left\langle K^{\prime}, E^{\prime}\right\rangle\right)\right)
$$

where $\operatorname{SCC}(\langle K, E\rangle)$ is the set of strongly connected components of $\langle K, E\rangle$ (which can be computed in linear time), and where $T\left(\left\langle K^{\prime}, E^{\prime}\right\rangle\right)$ is the transition system whose set of

\footnotetext{
${ }^{5}$ Formally the suspect game has been defined with a play as reference, and not a equivalence class. However, in this subsection, if $\pi$ and $\pi^{\prime}$ are equivalent, the games $\mathcal{H}(\mathcal{G}, \pi)$ and $\mathcal{H}\left(\mathcal{G}, \pi^{\prime}\right)$ are identical.
} 
states is $\left\{k \in K^{\prime} \mid(k, \mathrm{Agt}) \in W\left(\mathcal{G}, v\left(K^{\prime}\right)\right)\right\}$ and whose set of edges is

$$
\left\{\left(k, k^{\prime}\right) \in E^{\prime} \mid \exists\left(k, \operatorname{Agt}, m_{\text {Agt }}\right) \in W\left(\mathcal{G}, v\left(K^{\prime}\right)\right) . \operatorname{Tab}\left(k, m_{\text {Agt }}\right)=k^{\prime}\right\} .
$$

Notice that this set of edges is never empty, but $T\left(\left\langle K^{\prime}, E^{\prime}\right\rangle\right)$ might not be strongly connected anymore, so that this is really a recursive definition.

The recursive function SSG decomposes any (sub-)transition system of the game into a list of disjoint transition systems which all satisfy conditions (2)-(4) above.

So far the computation does not take into account the bounds for the payoffs of the players (lower bound $u^{A}$ and upper bound $w^{A}$ for player $A$ ). For each upper bound $w^{A}$, we assume condition $(\star)_{c}$ holds . In the particular case of a single Büchi objective for each player define by target $T_{A}$, this is simply done by setting $S^{A}=$ States $\backslash T_{A}$, if this player has to be losing (that is, if $w^{A}$ does not satisfy the Büchi objective). Now assuming we have found the appropriate set $S^{A}$, we define

$$
\text { Sol }=\mathrm{SSG}\left(\left\langle\bigcap_{A \in \mathrm{Agt}} S^{A}, \mathrm{Edg}^{\prime}\right\rangle\right) \cap\left\{\langle K, E\rangle \mid \forall A \in \operatorname{Agt} . u^{A} \lesssim v^{A}(K)\right\}
$$

where Edg' restricts Edg to $\bigcap_{A \in \mathrm{Agt}} S^{A}$.

We now show that the set Sol computes (in a sense that we make clear) the transition systems that are mentioned in Lemma 5.10).

Lemma 5.11. We suppose condition $(\star)$ holds. If $\langle K, E\rangle \in$ Sol then it satisfies conditions 1 to 4. Conversely, if $\langle K, E\rangle$ satisfies conditions 1 to 4, then there exists $\left\langle K^{\prime}, E^{\prime}\right\rangle \in$ Sol such that $\langle K, E\rangle \subseteq\left\langle K^{\prime}, E^{\prime}\right\rangle$.

Proof. Let $\langle K, E\rangle \in$ Sol. By definition of SSG, all ( $k$, Agt) for $k \in K$ are in $W(\mathcal{G}, v(K))$, and for all $\left(k, k^{\prime}\right) \in E$, there is a state $\left(k, \operatorname{Agt}, m_{\text {Agt }}\right)$ in $W(\mathcal{G}, v(K))$ such that $\operatorname{Tab}\left(k, m_{\text {Agt }}\right)=k^{\prime}$, and $\langle K, E\rangle$ is strongly connected. Also, for all $A, u^{A} \lesssim v^{A}(K)$ because Sol $\subseteq\{\langle K, E\rangle \mid$ $\left.u^{A} \lesssim v^{A}(K)\right\}$. Finally, for any $A \in \operatorname{Agt}, v^{A}(K) \lesssim w^{A}$ because the set $K$ is included in $S^{A}$.

Conversely, assume that $\langle K, E\rangle$ satisfies the conditions. We show that if $\langle K, E\rangle \subseteq$ $\left\langle K^{\prime}, E^{\prime}\right\rangle$ then there is $\left\langle K^{\prime \prime}, E^{\prime \prime}\right\rangle$ in $\operatorname{SSG}\left(\left\langle K^{\prime}, E^{\prime}\right\rangle\right)$ such that $\langle K, E\rangle \subseteq\left\langle K^{\prime \prime}, E^{\prime \prime}\right\rangle$. The proof is by induction on the size of $\left\langle K^{\prime}, E^{\prime}\right\rangle$.

The basic case is when $\left\langle K^{\prime}, E^{\prime}\right\rangle$ satisfies the conditions 2, 3, and 4; in that case, $\operatorname{SSG}\left(\left\langle K^{\prime}, E^{\prime}\right\rangle\right)=\left\{\left\langle K^{\prime}, E^{\prime}\right\rangle\right\}$, and by letting $\left\langle K^{\prime \prime}, E^{\prime \prime}\right\rangle=\left\langle K^{\prime}, E^{\prime}\right\rangle$ we get the expected result.

We now analyze the other case. There is a strongly connected component of $\left\langle K^{\prime}, E^{\prime}\right\rangle$, say $\left\langle K^{\prime \prime}, E^{\prime \prime}\right\rangle$, which contains $\langle K, E\rangle$, because $\langle K, E\rangle$ satisfies condition 2. We have $v^{A}(K) \lesssim A$ $v^{A}\left(K^{\prime \prime}\right)$ (because $K \subseteq K^{\prime \prime}$ and $\lesssim_{A}$ is monotonic) for every $A$, and thus $W(\mathcal{G}, v(K)) \subseteq$ $W\left(\mathcal{G}, v\left(K^{\prime \prime}\right)\right)$. This ensures that $T\left(\left\langle K^{\prime \prime}, E^{\prime \prime}\right\rangle\right)$ contains $\langle K, E\rangle$ as a subgraph. Since $\left\langle K^{\prime \prime}, E^{\prime \prime}\right\rangle$ is a subgraph of $\left\langle K^{\prime}, E^{\prime}\right\rangle$, the graph $T\left(\left\langle K^{\prime \prime}, E^{\prime \prime}\right\rangle\right)$ also is. We show that they are not equal, so that we can apply the induction hypothesis to $T\left(\left\langle K^{\prime \prime}, E^{\prime \prime}\right\rangle\right)$. For this, we exploit the fact that $\left\langle K^{\prime}, E^{\prime}\right\rangle$ does not satisfy one of conditions 2 to 4 ;

- first, if $\left\langle K^{\prime}, E^{\prime}\right\rangle$ is not strongly connected while $\left\langle K^{\prime \prime}, E^{\prime \prime}\right\rangle$ is, they cannot be equal;

- if there is some $k \in K^{\prime}$ such that ( $k$, Agt) is not in $W\left(\mathcal{G}, v\left(K^{\prime}\right)\right)$, then $k$ is not a vertex of $T\left(\left\langle K^{\prime \prime}, E^{\prime \prime}\right\rangle\right)$;

- if there some edge $\left(k, k^{\prime}\right)$ in $E^{\prime}$ such that there is no state $\left(k, \mathrm{Agt}, m_{\mathrm{Agt}}\right)$ in $W\left(\mathcal{G}, v\left(K^{\prime}\right)\right)$ such that $\operatorname{Tab}\left(k, m_{\mathrm{Agt}}\right)=k^{\prime}$, then the edge $\left(k, k^{\prime}\right)$ is not in $T\left(\left\langle K^{\prime \prime}, E^{\prime \prime}\right\rangle\right)$.

We then apply the induction hypothesis to $T\left(\left\langle K^{\prime \prime}, E^{\prime \prime}\right\rangle\right)$, and get the expected result. Now, because of condition 1, $u^{A} \lesssim v^{A}(K) \lesssim w^{A}$. Hence, due to the previous analysis, there exists 
$\left\langle K^{\prime}, E^{\prime}\right\rangle \in \mathrm{SSG}\left(\left\langle\bigcap_{A \in \mathrm{Agt}} S^{A}, \operatorname{Edg}^{\prime}\right\rangle\right)$ such that $\langle K, E\rangle \subseteq\left\langle K^{\prime}, E^{\prime}\right\rangle$. This concludes the proof of the lemma.

Lemma 5.12. Under assumptions $(\star)$, if for every $K$, the set $W(\mathcal{G}, v(K))$ can be computed in polynomial time, then the set Sol can also be computed in polynomial time.

Proof. Each recursive call to SSG applies to a decomposition in strongly connected components of the current transition system under consideration. Hence the number of recursive calls is bounded by $\mid$ States $\left.\right|^{2}$. Computing the decomposition in SCCs can be done in linear time. By assumption, each set $W(\mathcal{G}, v(K))$ can be computed in polynomial time. $S^{A}$ is obtained by removing the target of the losers (for $w^{A}$ ) from States. Hence globally we can compute Sol in polynomial time.

To conclude the algorithm, we need to check that condition 5 holds for one of the solutions $\langle K, E\rangle$ in Sol. It can be done in polynomial time by looking for a path in the winning region of Eve in $\mathcal{H}(\mathcal{G}, v(K))$ that reaches $K \times\{\mathrm{Agt}\}$ from $(s, \mathrm{Agt})$. The correctness of the algorithm is ensured by the fact that if some $\langle K, E\rangle$ satisfies the five conditions, there is a $\left\langle K^{\prime}, E^{\prime}\right\rangle$ in Sol with $K \subseteq K^{\prime}$ and $E \subseteq E^{\prime}$. Since $K \subseteq K^{\prime}$ implies $v^{A}(K) \lesssim_{A} v^{A}\left(K^{\prime}\right)$, the winning region of Eve in $\mathcal{H}\left(\mathcal{G}, v\left(K^{\prime}\right)\right)$ is larger than that $\mathcal{H}\left(\mathcal{G}, v\left(K^{\prime}\right)\right)$, which implies that the path from $(s, \mathrm{Agt})$ to $K \times\{\mathrm{Agt}\}$ is also a path from $(s, \mathrm{Agt})$ to $K^{\prime} \times\{\mathrm{Agt}\}$. Hence, $\left\langle K^{\prime}, E^{\prime}\right\rangle$ also satisfies condition 5 , and therefore the five expected conditions.

We have already mentioned that single Büchi objectives do satisfy the hypotheses $(\star)$. Furthermore, Lemma 5.9 shows that, given $v(K)$, one can compute the set $W(\mathcal{G}, v(K))$ as the winning region of a co-Büchi turn-based game, which can be done in polynomial time (this is argued at the beginning of the section). Therefore Lemma 5.12 and the subsequent analysis apply: this concludes the proof that the constrained NE existence problem for finite games with single Büchi objectives is in $\mathrm{P}$.

5.3.3. Hardness. We recall a possible proof of $\mathrm{P}$-hardness for the value problem, from which we will infer the other lower bounds. The circuit-value problem can be easily encoded into a deterministic turn-based game with Büchi objectives: a circuit (which we assume w.l.o.g. has only AND- and OR-gates) is transformed into a two-player turn-based game, where one player controls the AND-gates and the other player controls the OR-gates. We add self-loops on the leaves. Positive leaves of the circuit are the (Büchi) objective of the OR-player, and negative leaves are the (Büchi) objective of the AND-player. Then obviously, the circuit evaluates to true iff the OR-player has a winning strategy for satisfying his Büchi condition, which in turn is equivalent to the fact that there is an equilibrium with payoff 0 for the AND-player, by Proposition 3.2, We obtain P-hardness for the NE existence problem, using Proposition 3.4. the preference relations in the game constructed in Proposition 3.4 are Büchi objectives.

5.4. Co-Büchi objectives. The value problem for co-Büchi objectives is P-complete. We now prove that the constrained NE existence problem is in NP, and that the constrained NE existence problem and the NE existence problem are NP-hard. We therefore deduce:

Theorem 5.13. For finite games with single co-Büchi objectives, the NE existence problem and the constrained NE existence problem are NP-complete. 
The proof of this Theorem is very similar to that for safety objectives: instead of conjunction of reachability objectives, we need to deal with conjunction of Büchi objectives. Of course constructions and algorithms need to be adapted. That is what we present now.

5.4.1. Reduction to a conjunction of Büchi conditions. We assume that for every player $A$, $\Omega_{A}$ is a single co-Büchi objective $\Omega_{A}$ given by $T_{A}$. In the corresponding suspect game, we show that the goal of player Eve is equivalent to a conjunction of Büchi objectives. Let $L \subseteq$ Agt. In suspect game $\mathcal{H}(\mathcal{G}, L)$, we define several Büchi objectives as follows: for each $A \in L$, we define $T_{A}^{\prime}=T_{A} \times\{P \mid P \subseteq$ Agt $\} \cup$ States $\times\{P \mid A \notin P\}$, and we write $\Omega_{A}^{\prime}$ for the corresponding Büchi objective.

Lemma 5.14. A play $\rho$ is winning for Eve in $\mathcal{H}(\mathcal{G}, L)$ iff $\rho \in \bigcap_{A \in L} \Omega_{A}^{\prime}$.

Proof. Let $\rho$ be a play in $\mathcal{H}(\mathcal{G}, L)$, and assume it is winning for Eve. Then, for each $A \in \lambda(\rho) \cap L, \rho \notin \Omega_{A}$, which means that the target set $T_{A}$ is visited along $\operatorname{proj}_{1}(\rho)$, and therefore $T_{A}^{\prime}$ is visited infinitely often along $\rho$. If $A \notin \lambda(\rho)$, then a state $(s, P)$ with $A \notin P$ is visited infinitely often by $\rho$ : the target set $T_{A}^{\prime}$ is visited infinitely often. This implies that $\rho \in \bigcap_{A \in L} \Omega_{A}^{\prime}$.

Conversely let $\rho \in \bigcap_{A \in L} \Omega_{A}^{\prime}$. For every $A \in L, T_{A}^{\prime}$ is visited infinitely often by $\rho$. Then, either $T_{A}$ is visited infinitely often by $\operatorname{proj}_{1}(\rho)$ (which means that $\rho \notin \Omega_{A}$ ) or $A \notin \lambda(\rho)$. In particular, $\rho$ is a winning play for Eve in $\mathcal{H}(\mathcal{G}, L)$.

5.4.2. Algorithm for solving zero-sum games with a conjunction of Büchi objectives. We adapt the algorithm for conjunctions of reachability objectives (page 28) to conjunctions of Büchi objectives. Let $\mathcal{G}$ be a two-player turn-based game with a winning objective for Eve given as a conjunction of Büchi objectives $\Omega_{1}, \ldots, \Omega_{k}$. The idea is to construct a new game $\mathcal{G}^{\prime}$ which checks that each objective $\Omega_{i}$ is visited infinitely often. The vertices of $\mathcal{G}^{\prime}$ controlled by Eve and Adam are $V_{\exists}^{\prime}=V_{\exists} \times \llbracket 0, k \rrbracket$ and $V_{\forall}^{\prime}=V_{\forall} \times \llbracket 0, k \rrbracket$ respectively. There is a transition from $(v, k)$ to $\left(v^{\prime}, 0\right)$ iff there is a transition from $v$ to $v^{\prime}$ in the original game and for $0 \leq i<k$, there is a transition from $(v, i)$ to $\left(v^{\prime}, i+1\right)$ iff there is a transition from $v$ to $v^{\prime}$ in the original game and $v^{\prime} \in \Omega_{i+1}$. In $\mathcal{G}^{\prime}$, the objective for Eve is the Büchi objective $\Omega$ given by target set States $\times\{k\}$, where States $=V_{\exists} \cup V_{\forall}$ is the set of vertices of $\mathcal{G}$. It is clear that there is a winning strategy in $\mathcal{G}$ from $v_{0}$ for the conjunction of Büchi objectives $\Omega_{1}, \ldots, \Omega_{k}$ iff there is a winning strategy in $\mathcal{G}^{\prime}$ from $\left(v_{0}, 0\right)$ for the Büchi objective $\Omega$. The number of states of game $\mathcal{G}^{\prime}$ is $\mid$ States $^{\prime}|=|$ States $\mid \cdot k$, and the size of the transition table $\left|\operatorname{Tab}^{\prime}\right|=|\mathrm{Tab}| \cdot k$. Using the standard algorithm for turn-based Büchi objectives [13, which works in time $\mathcal{O}\left(\mid\right.$ States $\left.^{\prime}|\cdot| \mathrm{Tab}^{\prime} \mid\right)$, we obtain an algorithm for solving zero-sum games with a conjunction of Büchi objectives running in time $\mathcal{O}\left(k^{2} \cdot \mid\right.$ States $|\cdot|$ Tab $\left.\mid\right)$ (hence in polynomial time).

5.4.3. Algorithm. The algorithm is the same as for reachability objectives. Only the computation of the set of winning states in the suspect game is different. Since we just showed that this part can be done in polynomial time, the global algorithm still runs in (nondeterministic) polynomial time. 


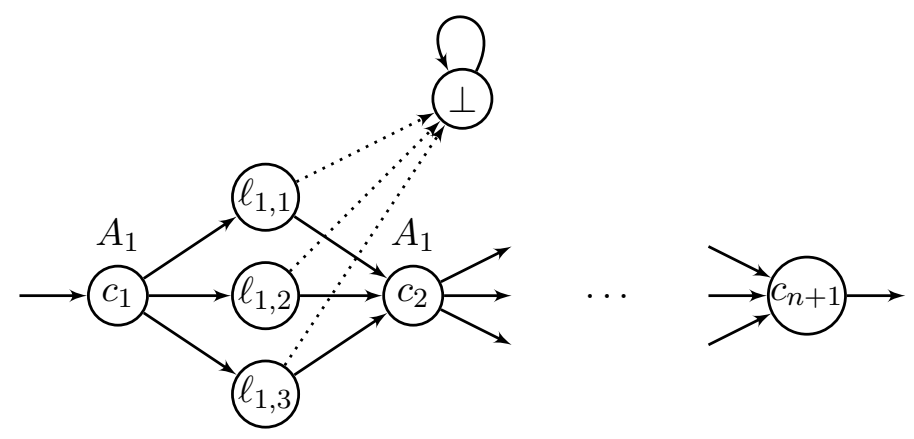

Figure 13. Module $M(\phi)$, where $\phi=c_{1} \wedge \cdots \wedge c_{n}$ and $c_{i}=\ell_{i, 1} \vee \ell_{i, 2} \vee \ell_{i, 3}$

5.4.4. Hardness. The hardness result for the constrained NE existence problem with coBüchi objectives was already proven in [40]. The idea is to encode an instance of 3SAT into a game with co-Büchi objectives. For completeness we describe the reduction below, and explain how it can be modified for proving NP-hardness of the NE existence problem.

Let us consider an instance $\phi=c_{1} \wedge \cdots \wedge c_{n}$ of SAT, where $c_{i}=\ell_{i, 1} \vee \ell_{i, 2} \vee \ell_{i, 3}$, and $\ell_{i, j} \in\left\{x_{k}, \neg x_{k} \mid 1 \leq k \leq p\right\}$. The game $\mathcal{G}$ is obtained from module $M(\phi)$ depicted on Figure 13, by joining the outgoing edge of $c_{n+1}$ to $c_{1}$. Each module $M(\phi)$ involves a set of players $B_{k}$, one for each variable $x_{k}$, and a player $A_{1}$. Player $A_{1}$ controls the clause states. Player $B_{k}$ control the literal states $\ell_{i, j}$ when $\ell_{i, j}=\neg x_{k}$, then having the opportunity to go to state $\perp$. There is no transition to $\perp$ for literals of the form $x_{k}$. In $M(\phi)$, assuming that the players $B_{k}$ will not play to $\perp$, then $A_{1}$ has a strategy that does not visit both $x_{k}$ and $\neg x_{k}$ for every $k$ if, and only if, formula $\phi$ is satisfiable. Finally, the co-Büchi objective of $B_{k}$ is given by $\left\{x_{k}\right\}$. In other terms, the aim of $B_{k}$ is to visit $x_{k}$ only a finite number of times. This way, in a Nash equilibrium, it cannot be the case that both $x_{k}$ and $\neg x_{k}$ are visited infinitely often: it would imply that $B_{k}$ loses but could improve her payoff by going to $\perp$ (actually, $\neg x_{k}$ should not be visited at all if $x_{k}$ is visited infinitely often). Therefore setting the objective of $A_{1}$ to $\{\perp\}$, there is a Nash equilibrium where she wins iff $\phi$ is satisfiable. This shows NP-hardness for the constrained NE existence problem.

For the NE existence problem, we use the transformation described in Section 3.4. We add an extra player $A_{2}$ to $\mathcal{G}$ and consider the game $\mathcal{G}^{\prime}=E\left(\mathcal{G}, A_{1}, A_{2}, \rho\right)$, where $\rho$ is a winning path for $A_{1}$. The objective of the players in $\mathcal{G}^{\prime}$ can be described by co-Büchi objectives: $A_{2}$ has to avoid seeing $T=\left\{s_{1}\right\}$ infinitely often and keep the same target for $A_{1}$. Applying Proposition 3.5, there is a Nash equilibrium in $\mathcal{G}^{\prime}$ if, and only if, there is one in $\mathcal{G}$ where $A_{1}$ wins, this shows NP-hardness for the NE existence problem.

5.5. Objectives given as circuits. The value problem is known to be PSPACE-complete for turn-based games and objectives given as circuits [27]. The transformation presented in the beginning of the section can be used to decide the value problem for finite concurrent games with a single circuit-objective, yielding PSPACE-completeness of the value problem in the case of finite concurrent games as well.

We now show that the (constrained) NE existence problem is also PSPACE-complete in this framework:

Theorem 5.15. For finite games with single objectives given as circuits, the NE existence problem and the constrained NE existence problem are PSPACE-complete. 
5.5.1. Reduction to a circuit objective. We assume the preference relation of each player $A \in$ Agt is given by a circuit $C_{A}$. Let $L \subseteq$ Agt. We define a Boolean circuit defining the winning condition of Eve in the suspect game $\mathcal{H}(\mathcal{G}, L)$.

We define for each player $A \in$ Agt and each set $P$ of players (such that States $\times P$ is reachable in $\mathcal{H}(\mathcal{G}, L)$ ), a circuit $D_{A, P}$ which outputs true for the plays $\rho$ with $\lambda(\rho)=P$ (i.e. whose states that are visited infinitely often are in States $\times\{P\})$, and whose value by $C_{A}$ is true. We do so by making a copy of the circuit $C_{A}$, adding $\mid$ States $\mid$ OR gates $g_{1} \cdots g_{\mid \text {States }} \mid$ and one AND gate $h$. There is an edge from $\left(s_{i}, P\right)$ to $g_{i}$ and from $g_{i-1}$ to $g_{i}$ if $i<\mid$ States $\mid$ then there is an edge from the output gate of $C_{A}$ to $h$ and from $h$ to the output gate of the new circuit. Inputs of $C_{A}$ are now the $\left(s, P\right.$ )'s (instead of the $s$ 's). The circuit $D_{A, P}$ is given on Figure 14.

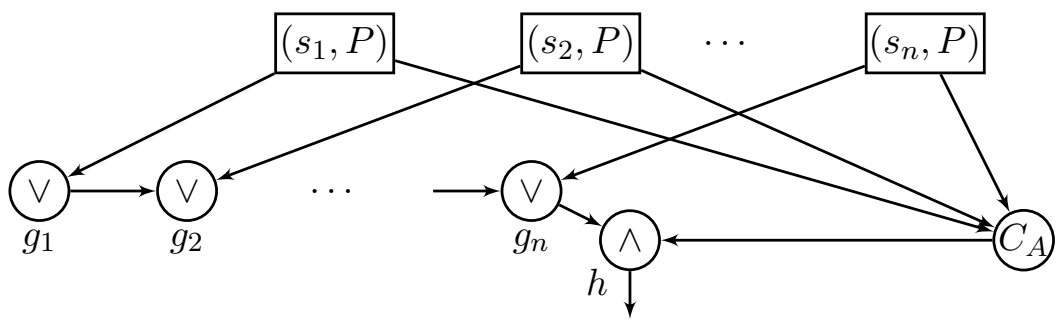

Figure 14. Circuit $D_{A, P}$

We then define a circuit $E_{A}$ which outputs true for the plays $\rho$ with $A \in \lambda(\rho)$ and whose output by $C_{A}$ is true. We do so by taking the disjunction of the circuits $D_{A, P}$. Formally, for each set of players $P$ such that States $\times P$ is reachable in the suspect game and $A \in P$, we include the circuit $D_{A, P}$ and writing $o_{A, P}$ for its output gate, we add OR gates so that there is an edge from $o_{A, P}$ to $g_{i}$ and from $g_{i}$ to $g_{i+1}$, and then from $g_{n+1}$ to the output gate.

Finally we define the circuit $F_{L}$, which outputs true for the plays $\rho$ such that there is no $A \in L$ such that $A \in \lambda(\rho)$ and the output of $\operatorname{proj}_{1}(\rho)$ by $C_{A}$ is true. This corresponds exactly to the plays that are winning for Eve in suspect game $\mathcal{H}(\mathcal{G}, L)$. We do so by negating the disjunction of all the circuits $E_{A}$ for $A \in L$.

The next lemma follows from the construction:

Lemma 5.16. A play $\rho$ is winning for Eve in $\mathcal{H}(\mathcal{G}, L)$ iff $\rho$ evaluates circuit $F_{L}$ to true.

We should notice that circuit $F_{L}$ has size polynomial in the size of $\mathcal{G}$, thanks to Proposition 4.8 .

5.5.2. Algorithm and complexity analysis. To solve the constrained NE existence problem we apply the same algorithm as for reachability objectives (see section 5.1). For complexity matters, the only difference stands in the computation of the set of winning states in the suspect game. Thanks to Lemma 5.16, we know it reduces to the computation of the set of winning states in a turn-based game with an objective given as a circuit (of polynomial-size). This can be done in PSPACE [27], which yields a PSPACE upper bound for the constrained NE existence problem (and therefore for the NE existence problem and the value problem - see Proposition 3.2). PSPACE-hardness of all problems follows from that of the value problem in turn-based games [27, and from Propositions 3.2 and 3.4 (we notice that the preference relations in the new games are easily definable by circuits). 
5.6. Rabin and parity objectives. The value problem is known to be NP-complete for Rabin conditions [18] and in UP $\cap$ co-UP for parity conditions [28].

We then notice that a parity condition is a Rabin condition with half as many pairs as the number of priorities: assume the parity condition is given by $p$ : States $\mapsto \llbracket 0, d \rrbracket$ with $d \in \mathbb{N}$; take for $i$ in $\llbracket 0, \frac{d}{2} \rrbracket, Q_{i}=p^{-1}\{2 i\}$ and $R_{i}=p^{-1}\{2 j+1 \mid j \geq i\}$. Then the Rabin objective $\left(Q_{i}, R_{i}\right)_{0 \leq i \leq \frac{d}{2}}$ is equivalent to the parity condition given by $p$.

We design an algorithm that solves the constrained NE existence problem in $\mathrm{P}_{\|}^{\mathrm{NP}}$ for Rabin objectives (see footnote 1 on page 4 for an informal definition of $\mathrm{P}_{\|}^{\mathrm{NP}}$ ).

Our algorithm heavily uses non-determinism (via the oracle). We then propose a deterministic algorithm which runs in exponential time, but will be useful in Section 5.7. This subsection ends with proving $\mathrm{P}_{\|}^{\mathrm{NP}}$-hardness of the constrained NE existence problem and $\mathrm{NE}$ existence problem for parity objectives. In the end, we will have proven the following theorem:

Theorem 5.17. For finite games with single objectives given as Rabin or parity conditions, the NE existence problem and the constrained NE existence problem are $\mathrm{P}_{\|}^{\mathrm{NP}}$-complete.

5.6.1. Reduction to a Streett game. We assume that the preference relation of each player $A \in$ Agt is given by the Rabin condition $\left(Q_{i, A}, R_{i, A}\right)_{i \in \llbracket 1, k_{A} \rrbracket}$. Let $L \subseteq$ Agt. In the suspect game $\mathcal{H}(\mathcal{G}, L)$, we define the Streett objective $\left(Q_{i, A}^{\prime}, R_{i, A}^{\prime}\right)_{i \in \llbracket 1, k_{A} \rrbracket, A \in L}$, where $Q_{i, A}^{\prime}=\left(Q_{i, A} \times\right.$ $\{P \mid A \in P\}) \cup($ States $\times\{P \mid A \notin P\})$ and $R_{i, A}^{\prime}=R_{i, A} \times\{P \mid A \in P\}$, and we write $\Omega_{L}$ for the corresponding set of winning plays.

Lemma 5.18. A play $\rho$ is winning for Eve in $\mathcal{H}(\mathcal{G}, L)$ iff $\rho \in \Omega_{L}$.

Proof. Assume $\rho$ is winning for Eve in $\mathcal{H}(\mathcal{G}, L)$. For all $A \in \lambda(\rho) \cap L, \operatorname{proj}_{1}(\rho)$ does not satisfy the Rabin condition given by $\left(Q_{i, A}, R_{i, A}\right)_{i \in \llbracket 1, k_{A} \rrbracket}$. For all $1 \leq i \leq k_{A}, \operatorname{Inf}\left(\operatorname{proj}_{1}(\rho)\right) \cap Q_{i, A}=\varnothing$ or $\operatorname{Inf}\left(\operatorname{proj}_{1}(\rho)\right) \cap R_{i, A} \neq \varnothing$. We infer that for all $1 \leq i \leq k_{A}, \operatorname{Inf}(\rho) \cap Q_{i, A}^{\prime}=\varnothing$ or $\operatorname{Inf}(\rho) \cap R_{i, A}^{\prime} \neq \varnothing$. Now, if $A \notin \lambda(\rho)$ then all $Q_{i, A}^{\prime}$ are seen infinitely often along $\rho$. Therefore for every $A \in L$, the Streett conditions $\left(Q_{i, A}^{\prime}, R_{i, A}^{\prime}\right)$ is satisfied along $\rho$ (that is, $\rho \in \Omega_{L}$ ).

Conversely, if the Streett condition $\left(Q_{i, A}^{\prime}, R_{i, A}^{\prime}\right)_{i \in \llbracket 1, k_{A} \rrbracket, A \in L}$ is satisfied along $\rho$, then either the Rabin condition $\left(Q_{i, A}, R_{i, A}\right)$ is not satisfied along $\operatorname{proj}_{1}(\rho)$ or $A \notin \lambda(\rho)$. This means that Eve is winning in $\mathcal{H}(\mathcal{G}, L)$.

5.6.2. Algorithm. We now describe a $\mathrm{P}_{\|}^{\mathrm{NP}}$ algorithm for solving the constrained NE existence problem in games where each player has a single Rabin objective. As in the previous cases, our algorithm relies on the suspect game construction.

Write $\mathcal{P}$ for the set of sets of players of Agt that appear as the second item of a state of $\mathcal{J}(\mathcal{G})$ :

$$
\mathcal{P}=\{P \subseteq \operatorname{Agt} \mid \exists s \in \text { States. }(s, P) \text { is a state of } \mathcal{J}(\mathcal{G})\} .
$$

Since $\mathcal{J}(\mathcal{G})$ has size polynomial, so has $\mathcal{P}$. Also, for any path $\rho, \lambda(\rho)$ is a set of $\mathcal{P}$. Hence, for a fixed $L$, the number of sets $\lambda(\rho) \cap L$ is polynomial. Now, as recalled on page 25. the winning condition for Eve is that the players in $\lambda(\rho) \cap L$ must be losing along $\operatorname{proj}_{1}(\rho)$ in $\mathcal{G}$ for their Rabin objective. We have seen that this can be seen as a Streett objective (Lemma 5.18). 
Now, deciding whether a state is winning in a turn-based game for a Streett condition can be decided in coNP [18]. Hence, given a state $s \in$ States and a set $L$, we can decide in coNP whether $s$ is winning for Eve in $\mathcal{H}(\mathcal{G}, L)$. This will be used as an oracle in our algorithm below.

Now, pick a set $P \subseteq$ Agt of suspects, i.e., for which there exists $(s, t) \in \operatorname{States}^{2}$ and $m_{\text {Agt }}$ s.t. $P=\operatorname{Susp}\left((s, t), m_{\text {Agt }}\right)$. Using the same arguments as in the proof of Proposition 4.8, it can be shown that $2^{|P|} \leq|\mathrm{Tab}|$, so that the number of subsets of $P$ is polynomial. Now, for each set $P$ of suspects and each $L \subseteq P$, write $w(L)$ for the size of the winning region of Eve in $\mathcal{H}(\mathcal{G}, L)$. Then the sum $\sum_{P \in \mathcal{P} \backslash\{\text { Agt }\}} \sum_{L \subseteq P} w(L)$ is at most $\mid$ States $|\times|$ Tab $\left.\right|^{2}$.

Assume that the exact value $M$ of this sum is known, and consider the following algorithm:

(1) for each $P \subseteq \mathcal{P} \backslash\{$ Agt $\}$ and each $L \subseteq P$, guess a set $W(L) \subseteq$ States, which we intend to be the exact winning region for Eve in $\mathcal{H}(\mathcal{G}, L)$.

(2) check that the sizes of those sets sum up to $M$;

(3) for each $s \notin W(L)$, check that Eve does not have a winning strategy from $s$ in $\mathcal{H}(\mathcal{G}, L)$. This can be checked in non-deterministic polynomial time, as explained above.

(4) guess a lasso-shaped path $\rho=\pi \cdot \tau^{\omega}$ in $\mathcal{H}(\mathcal{G}, L)$ starting from $(s, \operatorname{Agt})$, with $|\pi|$ and $|\tau|$ less than $\mid$ States $\left.\right|^{2}$ (following Proposition 3.1) visiting only states where the second item is Agt. This path can be seen as the outcome of some strategy of Eve when Adam obeys. For this path, we then check the following:

- along $\rho$, the sets of winning and losing players satisfy the original constraint (remember that we aim at solving the constrained NE existence problem);

- any deviation along $\rho$ leads to a state that is winning for Eve. In other terms, pick a state $h=\left(s\right.$, Agt, $\left.m_{\text {Agt }}\right)$ of Adam along $\rho$, and pick a successor $h^{\prime}=(t, P)$ of $h$ such that $t \neq \operatorname{Tab}\left(s, m_{\mathrm{Agt}}\right)$. Then the algorithm checks that $t \in W(L \cap P)$.

The algorithm accepts the input $M$ if it succeeds in finding the sets $W$ and the path $\rho$ such that all the checks are successful. This algorithm is non-deterministic and runs in polynomial time, and will be used as a second oracle.

We now show that if $M$ is exactly the sum of the $w(L)$, then the algorithm accepts $M$ if, and only if, there is a Nash equilibrium satisfying the constraint, i.e., if, and only if, Eve has a winning strategy from $(s, \mathrm{Agt})$ in $\mathcal{H}(\mathcal{G}, L)$.

First assume that the algorithm accepts $M$. This means that it is able, for each $L$, to find sets $W(L)$ of states whose complement does not intersect the winning region of $\mathcal{H}(\mathcal{G}, L)$. Since $M$ is assumed to be the exact sum of $w(L)$ and the size of the sets $W(L)$ sum up to $M$, we deduce that $W(L)$ is exactly the winning region of Eve in $\mathcal{H}(\mathcal{G}, L)$. Now, since the algorithm accepts, it is also able to find a (lasso-shaped) path $\rho$ only visiting states having Agt as the second component. This path has the additional property that any "deviation" from a state of Adam along this path ends up in a state that is winning for Eve for players in $L \cap P$, where $P$ is the set of suspects for the present deviation. This way, if during $\rho$, Adam deviates to a state $(t, P)$, then Eve will have a strategy to ensure that along any subsequent play, the objectives of players in $L \cap P$ (in $\mathcal{G}$ ) are not fulfilled, so that along any run $\rho^{\prime}$, the players in $L \cap \lambda\left(\rho^{\prime}\right)$ are losing for their objectives in $\mathcal{G}$, so that Eve wins in $\mathcal{H}(\mathcal{G}, L)$.

Conversely, assume that there is a Nash equilibrium satisfying the constraint. Following Proposition 3.1, we assume that the outcome of the corresponding strategy profile has the form $\pi \cdot \tau^{\omega}$. From Lemma 4.4, there is a winning strategy for Eve in $\mathcal{H}(\mathcal{G}, L)$ whose 
outcome when Adam obeys follows the outcome of the Nash equilibrium. As a consequence, the outcome when Adam obeys is a path $\rho$ that the algorithm can guess. Indeed, it must satisfy the constraints, and any deviation from $\rho$ with set of suspects $P$ ends in a state where Eve wins for the winning condition of $\mathcal{H}(\mathcal{G}, L)$, hence also for the winning condition of $\mathcal{H}(\mathcal{G}, L \cap P)$, since any path $\rho^{\prime}$ visiting $(t, P)$ has $\lambda\left(\rho^{\prime}\right) \subseteq P$.

Finally, our global algorithm is as follows: we run the first oracle for all the states and all the sets $L$ that are subsets of a set of suspects (we know that there are polynomially many such inputs). We also run the second algorithm on all the possible values for $M$, which are also polynomially many. Now, from the answers of the first oracle, we compute the exact value $M$, and return the value given by the second on that input. This algorithm runs in $\mathrm{P}_{\|}^{\mathrm{NP}}$ and decides the constrained NE existence problem.

5.6.3. Deterministic algorithm. In the next section we will need a deterministic algorithm to solve games with objectives given as deterministic Rabin automata. We therefore present it right now. The deterministic algorithm works by successively trying all the possible payoffs, there are $2^{|\mathrm{Agt}|}$ of them. Then it computes the winning strategies of the suspect game for that payoff. In [25] an algorithm for Streett games is given, which works in time $\mathcal{O}\left(n^{k} \cdot k !\right)$, where $n$ is the number of vertices in the game, and $k$ the size of the Streett condition. The algorithm has to find, in the winning region of Eve in $\mathcal{J}(\mathcal{G})$, a lasso that satisfies the Rabin winning conditions of the winners and do not satisfy whose of the losers. To do so it tries all the possible choices of elementary Rabin condition that are satisfied to make the players win, there are at most $\prod_{A \in \text { Agt }} k_{A}$ possible choices. And for the losers, we try the possible choices for whether $Q_{i, A}$ is visited of not, there are $\prod_{A \in \mathrm{Agt}} 2^{k_{A}}$ such choices. It then looks for a lasso cycle that, when $A$ is a winner, does not visit $Q_{i_{A}, A}$ and visits $R_{i_{A}, A}$, and when $A$ is a loser, visits $R_{i_{A}, A}$ when it has to, or does not visit $Q_{i_{A}, A}$. This is equivalent to finding a path satisfying a conjunction of Büchi conditions and can be done in polynomial time $\mathcal{O}\left(n \times \sum_{A \in \mathrm{Agt}} k_{A}\right)$. The global algorithm works in time

$$
\mathcal{O}\left(2^{|\mathrm{Agt}|} \cdot\left(|\mathrm{Tab}|^{3 \sum_{A} k_{A}} \cdot\left(\sum_{A} k_{A}\right) !+\left(\prod_{A \in \mathrm{Agt}} k_{A} \cdot 2^{k_{A}}\right) \cdot|\mathrm{Tab}|^{3} \cdot \sum_{A} k_{A}\right)\right)
$$

Notice that the exponential does not come from the size of the graph but from the number of agents and the number of elementary Rabin conditions, this will be important when in the next subsection we will reuse the algorithm on a game structure whose size is exponential.

5.6.4. $\mathrm{P}_{\|}^{\mathrm{NP}}$-hardness. We now prove $\mathrm{P}_{\|}^{\mathrm{NP}}$-hardness of the (constrained) NE existence problem in the case of parity objectives. The main reduction is an encoding of the $\oplus$ SAT problem, where the aim is to decide whether the number of satisfiable instances among a set of formulas is even. This problem is known to be complete for $\mathrm{P}_{\|}^{\mathrm{NP}}[22]$.

Before tackling the whole reduction, we first develop some preliminaries on single instances of SAT, inspired from [12]. Let us consider an instance $\phi=c_{1} \wedge \cdots \wedge c_{n}$ of SAT, where $c_{i}=\ell_{i, 1} \vee \ell_{i, 2} \vee \ell_{i, 3}$, and $\ell_{i, j} \in\left\{x_{k}, \neg x_{k} \mid 1 \leq k \leq p\right\}$. With $\phi$, we associate a three-player game $N(\phi)$, depicted on Figure 15 (where the first state of $N(\phi)$ is controlled by $A_{1}$, and 

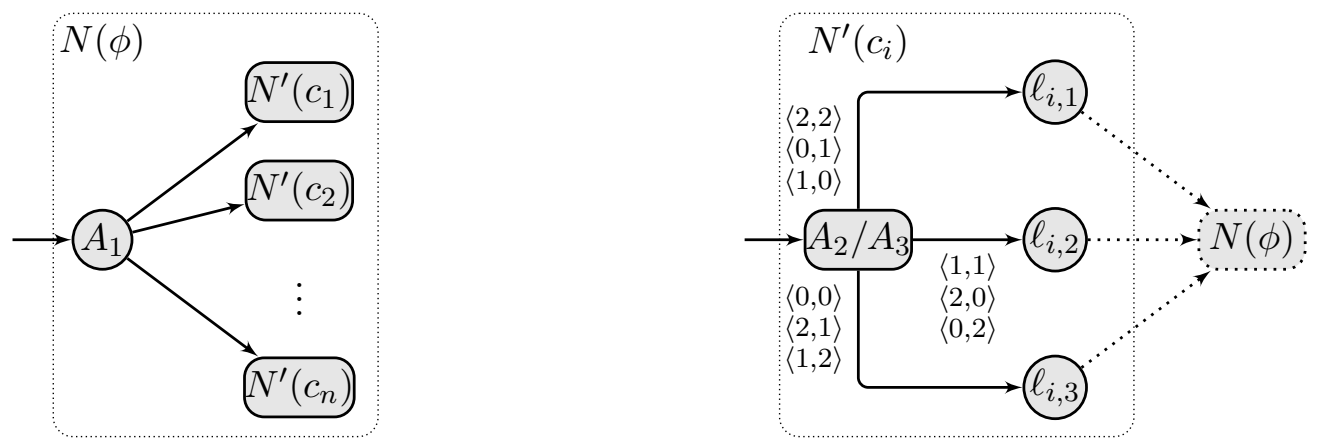

Figure 15. The game $N(\phi)$ (left), where $N^{\prime}\left(c_{i}\right)$ is the module on the right.

the first state of each $N^{\prime}\left(c_{j}\right)$ is concurrently controlled by $A_{2}$ and $\left.A_{3}\right)$. For each variable $x_{j}$, players $A_{2}$ and $A_{3}$ have the following target sets:

$$
T_{2 j}^{A_{2}}=\left\{x_{j}\right\} \quad T_{2 j+1}^{A_{2}}=\left\{\neg x_{j}\right\} \quad T_{2 j+1}^{A_{3}}=\left\{x_{j}\right\} \quad T_{2 j}^{A_{3}}=\left\{\neg x_{j}\right\}
$$

This construction enjoys interesting properties, given by the following lemma:

Lemma 5.19. If the formula $\phi$ is not satisfiable, then there is a strategy for player $A_{1}$ in $N(\phi)$ such that players $A_{2}$ and $A_{3}$ lose. If the formula $\phi$ is satisfiable, then for any strategy profile $\sigma_{\mathrm{Agt}}$, one of $A_{2}$ and $A_{3}$ can change her strategy and win.

Proof. We begin with the first statement, assuming that $\phi$ is not satisfiable and defining the strategy for $A_{1}$. With a history $h$ in $N(\phi)$, we associate a valuation $v^{h}:\left\{x_{k} \mid k \in[1, p]\right\} \rightarrow$ $\{\top, \perp\}$ (where $p$ is the number of distinct variables in $\phi$ ), defined as follows:

$$
v^{h}\left(x_{k}\right)=\top \Leftrightarrow \exists m . h_{m}=x_{k} \wedge \forall m^{\prime}>m . h_{m^{\prime}} \neq \neg x_{k} \quad \text { for all } k \in[1, p]
$$

We also define $v^{h}\left(\neg x_{k}\right)=\neg v^{h}\left(x_{k}\right)$. Under this definition, $v^{h}\left(x_{k}\right)=\top$ if the last occurrence of $x_{k}$ or $\neg x_{k}$ along $h$ was $x_{k}$. We then define a strategy $\sigma_{1}$ for player $A_{1}$ : after a history $h$ ending in an $A_{1}$-state, we require $\sigma_{1}(h)$ to go to $N^{\prime}\left(c_{i}\right)$ for some $c_{i}$ (with least index, say) that evaluates to false under $v^{h}$ (such a $c_{i}$ exists since $\phi$ is not satisfiable). This strategy enforces that if $h \cdot \sigma_{1}(h) \cdot \ell_{i, j}$ is a finite outcome of $\sigma_{1}$, then $v^{h}\left(\ell_{i, j}\right)=\perp$, because $A_{1}$ has selected a clause $c_{i}$ whose literals all evaluate to $\perp$. Moreover, $v^{h \cdot \sigma_{1}(h) \cdot \ell_{i, j}}\left(\ell_{i, j}\right)=\top$, so that for each $j$, any outcome of $\sigma_{1}$ will either alternate between $x_{k}$ and $\neg x_{k}$ (hence visit both of them infinitely often), or no longer visit any of them after some point. Hence both $A_{2}$ and $A_{3}$ lose.

We now prove the second statement. Let $v$ be a valuation under which $\phi$ evaluates to true, and $\sigma_{\text {Agt }}$ be a strategy profile. From $\sigma_{A_{2}}$ and $\sigma_{A_{3}}$, we define two strategies $\sigma_{A_{2}}^{\prime}$ and $\sigma_{A_{3}}^{\prime}$. Consider a finite history $h$ ending in the first state of $N^{\prime}\left(c_{i}\right)$, for some $i$. Pick a literal $\ell_{i, j}$ of $c_{i}$ that is true under $v$ (the one with least index, say). We set

$$
\sigma_{A_{2}}^{\prime}(h)=\left[j-\sigma_{A_{3}}(h)(\bmod 3)\right] \quad \sigma_{A_{3}}^{\prime}(h)=\left[j-\sigma_{A_{2}}(h)(\bmod 3)\right] .
$$

It is easily checked that, when $\sigma_{A_{2}}$ and $\sigma_{A_{3}}^{\prime}$ (or $\sigma_{A_{2}}^{\prime}$ and $\sigma_{A_{3}}$ ) are played simultaneously in the first state of some $N^{\prime}\left(c_{i}\right)$, then the game goes to $\ell_{i, j}$. Thus under those strategies, any visited literal evaluates to true under $v$, which means that at most one of $x_{k}$ and $\neg x_{k}$ is visited (infinitely often). Hence one of $A_{2}$ and $A_{3}$ is winning, which proves our claim. 
We now proceed by encoding an instance

$$
\begin{gathered}
\exists x_{1}^{1}, \ldots x_{k}^{1} \cdot \phi^{1}\left(x_{1}^{1}, \ldots, x_{k}^{1}\right) \\
\ldots \\
\exists x_{1}^{m}, \ldots x_{k}^{m} \cdot \phi^{m}\left(x_{1}^{m}, \ldots, x_{k}^{m}\right)
\end{gathered}
$$

of $\oplus$ SAT into a parity game. The game involves the three players $A_{1}, A_{2}$ and $A_{3}$ of the game $N(\phi)$ defined above, and it will contain a copy of $N\left(\phi^{r}\right)$ for each $1 \leq r \leq m$. The objectives of $A_{2}$ and $A_{3}$ are the unions of their objectives in each $N\left(\phi^{r}\right)$, e.g. $p^{A_{2}}\left(x_{j}^{1}\right)=$ $p^{A_{2}}\left(x_{j}^{2}\right)=\cdots=p^{A_{m}}\left(x_{j}^{m}\right)=2 j$.

For each such $r$, the game will also contain a copy of the game $M\left(\phi^{r}\right)$ depicted on Figure 13, Each game $M\left(\phi^{r}\right)$ involves an extra set of players $B_{k}^{r}$, one for each variable $x_{k}^{r}$. As we have seen in Section 5.4, in a Nash equilibrium, it cannot be the case that both $x_{k}^{r}$ and $\neg x_{k}^{r}$ are visited infinitely often.

In order to test the parity of the number of satisfiable formulas, we then define two families of modules, depicted on Figure [16 to 19. Finally, the whole game $\mathcal{G}$ is depicted on Figure 20. In that game, the objective of $A_{1}$ is to visit infinitely often the initial state init.

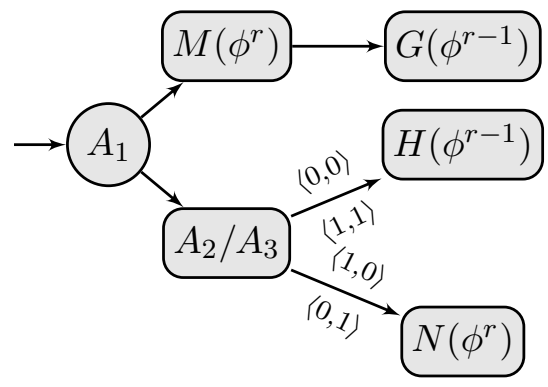

Figure 16. Module $H\left(\phi^{r}\right)$ for $r \geq 2$

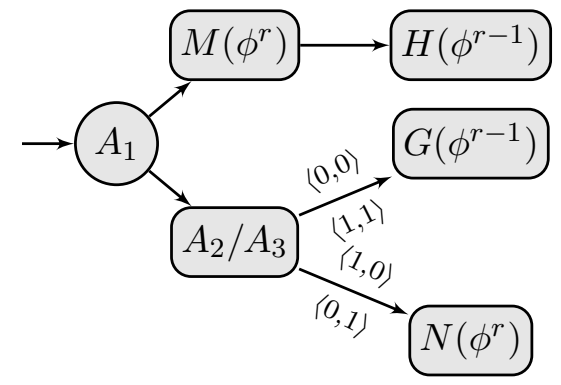

Figure 17. Module $G\left(\phi^{r}\right)$ for $r \geq 2$

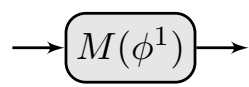

FIG. 18. Module $H\left(\phi^{1}\right)$

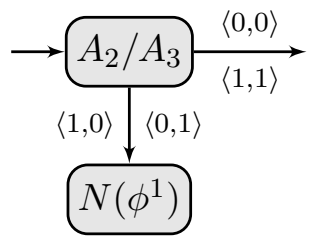

FIG. 19. Module $G\left(\phi^{1}\right)$

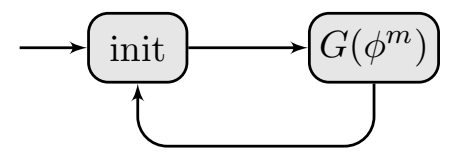

FIG. 20. The game $\mathcal{G}$

Lemma 5.20. There is a Nash equilibrium in the game $\mathcal{G}$ where $A_{2}$ and $A_{3}$ lose and $A_{1}$ wins if, and only if, the number of satisfiable formulas is even.

Proof. Assume that there is a Nash equilibrium in $\mathcal{G}$ where $A_{1}$ wins and both $A_{2}$ and $A_{3}$ lose. Let $\rho$ be its outcome. As already noted, if $\rho$ visits module $M\left(\phi^{r}\right)$ infinitely often, then it cannot be the case that both $x_{k}^{r}$ and $\neg x_{k}^{r}$ are visited infinitely often in $M\left(\phi^{r}\right)$, as otherwise $B_{k}^{r}$ would be losing and have the opportunity to improve her payoff. This implies that $\phi^{r}$ is satisfiable. Similarly, if $\rho$ visits infinitely often the states of $H\left(\phi^{r}\right)$ or $G\left(\phi^{r}\right)$ that is controlled by $A_{2}$ and $A_{3}$, then it must be the case that $\phi^{r}$ is not satisfiable, since from Lemma 5.19 this would imply that $A_{2}$ or $A_{3}$ could deviate and improve her payoff by going to $N\left(\phi^{r}\right)$. 
We now show by induction on $r$ that if $\rho$ goes infinitely often in module $G\left(\phi^{r}\right)$ then $\#\left\{j \leq r \mid \phi^{r}\right.$ is satisfiable $\}$ is even, and that (if $n>1$ ) this number is odd if $\rho$ goes infinitely in module $H\left(\phi^{r}\right)$.

When $r=1$, since $H\left(\phi^{1}\right)$ is $M\left(\phi^{1}\right), \phi^{1}$ is satisfiable, as noted above. Similarly, if $\rho$ visits $G\left(\phi^{1}\right)$ infinitely often, it also visits its $A_{2} / A_{3}$-state infinitely often, so that $\phi^{1}$ is not satisfiable. This proves the base case.

Assume that the result holds up to some $r-1$, and assume that $\rho$ visits $G\left(\phi^{r}\right)$ infinitely often. Two cases may occur:

- it can be the case that $M\left(\phi^{r}\right)$ is visited infinitely often, as well as $H\left(\phi^{r-1}\right)$. Then $\phi^{r}$ is satisfiable, and the number of satisfiable formulas with index less than or equal to $r-1$ is odd. Hence the number of satisfiable formulas with index less than or equal to $r$ is even.

- it can also be the case that the state $A_{2} / A_{3}$ of $G\left(\phi^{r}\right)$ is visited infinitely often. Then $\phi^{r}$ is not satisfiable. Moreover, since $A_{1}$ wins, the play will also visit $G\left(\phi^{r-1}\right)$ infinitely often, so that the number of satisfiable formulas with index less than or equal to $r$ is even.

If $\rho$ visits $H\left(\phi^{r}\right)$ infinitely often, using similar arguments we prove that the number of satisfiable formulas with index less than or equal to $r$ is odd.

To conclude, since $A_{1}$ wins, the play visits $G\left(\phi^{m}\right)$ infinitely often, so that the total number of satisfiable formulas is even.

Conversely, assume that the number of satisfiable formulas is even. We build a strategy profile, which we prove is a Nash equilibrium in which $A_{1}$ wins, and $A_{2}$ and $A_{3}$ lose. The strategy for $A_{1}$ in the initial states of $H\left(\phi^{r}\right)$ and $G\left(\phi^{r}\right)$ is to go to $M\left(\phi^{r}\right)$ when $\phi^{r}$ is satisfiable, and to state $A_{2} / A_{3}$ otherwise. In $M\left(\phi^{r}\right)$, the strategy is to play according to a valuation satisfying $\phi^{r}$. In $N\left(\phi^{r}\right)$, it follows a strategy along which $A_{2}$ and $A_{3}$ lose (this exists according to Lemma 5.19). This defines the strategy for $A_{1}$. Then $A_{2}$ and $A_{3}$ are required to always play the same move, so that the play never goes to some $N\left(\phi^{r}\right)$. In $N\left(\phi^{r}\right)$, they can play any strategy (they lose anyway, whatever they do). Finally, the strategy of $B_{k}^{r}$ never goes to $\perp$.

We now explain why this is the Nash equilibrium we are after. First, as $A_{1}$ plays according to fixed valuations for the variables $x_{k}^{r}$, either $B_{k}^{r}$ wins or she does not have the opportunity to go to $\perp$. It remains to prove that $A_{1}$ wins, and that $A_{2}$ and $A_{3}$ lose and cannot improve (individually). To see this, notice that between two consecutive visits to init, exactly one of $G\left(\phi^{r}\right)$ and $H\left(\phi^{r}\right)$ is visited. More precisely, it can be observed that the strategy of $A_{1}$ enforces that $G\left(\phi^{r}\right)$ is visited if $\#\left\{r<r^{\prime} \leq m \mid \phi^{r^{\prime}}\right.$ is satisfiable $\}$ is even, and that $H\left(\phi^{r}\right)$ is visited otherwise. Then if $H\left(\phi_{1}\right)$ is visited, the number of satisfiable formulas with index between 2 and $m$ is odd, so that $\phi_{1}$ is satisfiable and $A_{1}$ can return to init. If $G\left(\phi^{1}\right)$ is visited, an even number of formulas with index between 2 and $m$ is satisfiable, and $\phi^{1}$ is not. Hence $A_{1}$ has a strategy in $N\left(\phi^{1}\right)$ to make $A_{2}$ and $A_{3}$ lose, so that $A_{2}$ and $A_{3}$ cannot improve their payoffs.

This proves hardness for the constrained NE existence problem with parity objectives. For the NE existence problem, we use the construction of Section 3.4, but since it can only be used to get rid of constraint of the type " $A_{1}$ is winning", we add to the game two players, $A_{4}$ and $A_{5}$, whose objectives are opposite to $A_{2}$ and $A_{3}$ respectively, and one player $A_{6}$ that will be playing matching-penny games. The objectives for $A_{4}$ and $A_{5}$ are definable by parity objectives, by adding 1 to all the priorities. Then, we consider game $\mathcal{G}^{\prime}=E\left(E\left(E\left(\mathcal{G}, A_{1}, A_{6}, \rho_{1}\right), A_{4}, A_{6}, \rho_{4}\right), A_{5}, A_{6}, \rho_{5}\right)$ where $\rho_{1}, \rho_{4}$ and $\rho_{5}$ are winning paths 
for $A_{1}, A_{4}$ and $A_{5}$ respectively. Thanks to Proposition 3.5, there is a Nash equilibrium in $\mathcal{G}^{\prime}$ if, and only if, there is a Nash equilibrium in $\mathcal{G}$ where $A_{1}$ wins and $A_{2}$ and $A_{3}$ lose. We deduce $\mathrm{P}_{\|}^{\mathrm{NP}}$-hardness for the NE existence problem with parity objectives.

5.7. Objectives given as deterministic Rabin automata. In order to find Nash equilibria when objectives are given as deterministic Rabin automata, we first define the notion of game simulation, which we show has the property that when $\mathcal{G}^{\prime}$ game-simulates $\mathcal{G}$, then a Nash equilibrium in the latter game gives rise to a Nash equilibrium in the former one.

We then define the product of a game with automata (defining the objectives of the players), and show that it game-simulates the original game. This reduces the case of games with objectives are defined as Rabin automata to games with Rabin objectives, which we handled at the previous section; the resulting algorithm is in EXPTIME. We then show a PSPACE lower bound for the problem in the case of objectives given as deterministic Büchi automata. This proves the following theorem:

Theorem 5.21. For finite games with single objectives given as deterministic Rabin automata or deterministic Büchi automata, the NE existence problem and the constrained NE existence problem are in EXPTIME and PSPACE-hard.

It must be noticed that game simulation can be used in other contexts: in particular, in 4] (where we introduced this notion), it is shown that a region-based abstraction of timed games game simulates its original timed game, which provides a way of computing Nash equilibria in timed games.

5.7.1. Game simulation. We define game simulation and show how that can be used to compute Nash equilibria. We then apply it to objectives given as deterministic Rabin automata.

Definition 5.22. Consider two games $\mathcal{G}=\left\langle\right.$ States, Agt, Act, Mov, Tab, $\left.\left(\precsim_{A}\right)_{A \in \text { Agt }}\right\rangle$ and $\mathcal{G}^{\prime}=\left\langle\right.$ States $^{\prime}$, Agt, Act $\left.^{\prime}, \mathrm{Mov}^{\prime}, \mathrm{Tab}^{\prime},\left(\precsim_{A}^{\prime}\right)_{A \in \mathrm{Agt}}\right\rangle$ with the same set Agt of players. A relation $\triangleleft \subseteq$ States $\times$ States $^{\prime}$ is a game simulation if $s \triangleleft s^{\prime}$ implies that for each move $m_{\text {Agt }}$ in $\mathcal{G}$ there exists a move $m_{\text {Agt }}^{\prime}$ in $\mathcal{G}^{\prime}$ such that:

(1) $\operatorname{Tab}\left(s, m_{\text {Agt }}\right) \triangleleft \operatorname{Tab}^{\prime}\left(s^{\prime}, m_{\text {Agt }}^{\prime}\right)$, and

(2) for each $t^{\prime} \in$ States $^{\prime}$ there exists $t \in$ States with $t \triangleleft t^{\prime}$ and

$\operatorname{Susp}\left(\left(s^{\prime}, t^{\prime}\right), m_{\mathrm{Agt}}^{\prime}\right) \subseteq \operatorname{Susp}\left((s, t), m_{\mathrm{Agt}}\right)$.

If $\triangleleft$ is a game simulation and $\left(s_{0}, s_{0}^{\prime}\right) \in \triangleleft$, we say that $\mathcal{G}^{\prime}$ game-simulates (or simply simulates) $\mathcal{G}$. When there are two paths $\rho$ and $\rho^{\prime}$ such that $\rho_{=i} \triangleleft \rho_{=i}^{\prime}$ for all $i \in \mathbb{N}$, we will simply write $\rho \triangleleft \rho^{\prime}$.

A game simulation $\triangleleft$ is preference-preserving from $\left(s_{0}, s_{0}^{\prime}\right) \in$ States $\times$ States $^{\prime}$ if for all $\rho_{1}, \rho_{2} \in \operatorname{Play}_{\mathcal{G}}\left(s_{0}\right)$ and $\rho_{1}^{\prime}, \rho_{2}^{\prime} \in \operatorname{Play}_{\mathcal{G}^{\prime}}\left(s_{0}^{\prime}\right)$ with $\rho_{1} \triangleleft \rho_{1}^{\prime}$ and $\rho_{2} \triangleleft \rho_{2}^{\prime}$, for all $A \in$ Agt it holds that $\rho_{1} \precsim A \rho_{2}$ iff $\rho_{1}^{\prime} \precsim_{A}^{\prime} \rho_{2}^{\prime}$.

As we show now, Nash equilibria are preserved by game simulation, in the following sense:

Proposition 5.23. Let $\mathcal{G}=\left\langle\right.$ States, Agt, Act, Mov, Tab, $\left.\left(\precsim_{A}\right)_{A \in \text { Agt }}\right\rangle$ and $\mathcal{G}^{\prime}=\left\langle\right.$ States $^{\prime}$, Agt, $\left.\mathrm{Act}^{\prime}, \mathrm{Mov}^{\prime}, \mathrm{Tab}^{\prime},\left(\precsim_{A}^{\prime}\right)_{A \in \mathrm{Agt}}\right\rangle$ be two games involving the same set of players. Fix two states $s_{0}$ and $s_{0}^{\prime}$ in $\mathcal{G}$ and $\mathcal{G}^{\prime}$ respectively, and let $\triangleleft$ be a preference-preserving game simulation 
from $\left(s_{0}, s_{0}^{\prime}\right)$. If there exists a Nash equilibrium $\sigma_{\mathrm{Agt}}$ in $\mathcal{G}$ from $s_{0}$, then there exists a Nash equilibrium $\sigma_{\text {Agt }}^{\prime}$ in $\mathcal{G}^{\prime}$ from $s_{0}^{\prime}$ with $\operatorname{Out}_{\mathcal{G}}\left(s_{0}, \sigma_{\mathrm{Agt}}\right) \triangleleft \operatorname{Out}_{\mathcal{G}^{\prime}}\left(s_{0}^{\prime}, \sigma_{\mathrm{Agt}}^{\prime}\right)$.

Proof. We fix a strategy profile $\sigma_{\mathrm{Agt}}$ in $\mathcal{G}$ and $\rho$ the outcome of $\sigma_{\mathrm{Agt}}$ from $s_{0}$. We derive a strategy profile $\sigma_{\text {Agt }}^{\prime}$ in $\mathcal{G}^{\prime}$ and its outcome $\rho^{\prime}$ from $s_{0}^{\prime}$, such that:

(a) for every $\bar{\rho}^{\prime} \in \operatorname{Play}_{\mathcal{G}^{\prime}}\left(s_{0}^{\prime}\right)$, there exists $\bar{\rho} \in \operatorname{Play}_{\mathcal{G}}\left(s_{0}\right)$ s.t. $\bar{\rho} \triangleleft \bar{\rho}^{\prime}$ and $\operatorname{Susp}\left(\bar{\rho}^{\prime}, \sigma_{\text {Agt }}^{\prime}\right) \subseteq$ $\operatorname{Susp}\left(\bar{\rho}, \sigma_{\mathrm{Agt}}\right)$

(b) $\rho \triangleleft \rho^{\prime}$.

Assume we have done the construction, and that $\sigma_{\text {Agt }}$ is a Nash equilibrium in $\mathcal{G}$. We prove that $\sigma_{\text {Agt }}^{\prime}$ is a Nash equilibrium in $\mathcal{G}^{\prime}$. Towards a contradiction, assume that some player $A$ has a strategy $\bar{\sigma}_{A}^{\prime}$ in $\mathcal{G}^{\prime}$ such that $\bar{\rho}^{\prime} \mathscr{L}_{A} \rho^{\prime}$, where $\bar{\rho}^{\prime}=\operatorname{Out}_{\mathcal{G}^{\prime}}\left(s^{\prime}, \sigma_{\text {Agt }}^{\prime}\left[A \mapsto \bar{\sigma}_{A}^{\prime}\right]\right)$. Note that $A \in \operatorname{Susp}\left(\bar{\rho}^{\prime}, \sigma_{\text {Agt }}^{\prime}\right)$. Applying (a) above, there exists $\bar{\rho} \in \operatorname{Play}_{\mathcal{G}}\left(s_{0}\right)$ such that $\bar{\rho} \triangleleft \bar{\rho}^{\prime}$ and $\operatorname{Susp}\left(\bar{\rho}^{\prime}, \sigma_{\text {Agt }}^{\prime}\right) \subseteq \operatorname{Susp}\left(\bar{\rho}, \sigma_{\text {Agt }}\right)$. In particular, $A \in \operatorname{Susp}\left(\bar{\rho}, \sigma_{\text {Agt }}\right)$, and there exists a strategy $\bar{\sigma}_{A}$ for $A$ such that $\bar{\rho}=\operatorname{Out}_{\mathcal{G}}\left(s_{0}, \sigma_{\mathrm{Agt}}[A \mapsto \bar{\sigma}]\right.$ ). As $\rho \triangleleft \rho^{\prime}$ (by $\left.([\mathrm{b})]\right)$ and $\triangleleft$ is preference-preserving from $\left(s_{0}, s_{0}^{\prime}\right), \bar{\rho} \not L_{A} \rho$, which contradicts the fact that $\sigma_{\text {Agt }}$ is a Nash equilibrium. Hence, $\sigma_{\text {Agt }}^{\prime}$ is a Nash equilibrium in $\mathcal{G}^{\prime}$ from $s_{0}^{\prime}$.

It remains to show how we construct $\sigma_{\text {Agt }}^{\prime}$ (and $\rho^{\prime}$ ). We first build $\rho^{\prime}$ inductively, and define $\sigma_{\text {Agt }}^{\prime}$ along that path.

- Initially, we let $\rho_{=0}^{\prime}=s_{0}^{\prime}$. Since $\triangleleft$ is a game simulation containing $\left(s_{0}, s_{0}^{\prime}\right)$, we have $s_{0} \triangleleft s_{0}^{\prime}$, and there is a move $m_{\text {Agt }}^{\prime}$ associated with $\sigma_{\text {Agt }}\left(s_{0}\right)$ satisfying the conditions of Definition 5.22. Then $\rho_{=0} \triangleleft \rho_{=0}^{\prime}$, and $\operatorname{Susp}\left(\rho_{=0}^{\prime}, \sigma_{\text {Agt }}^{\prime}\left(\rho_{=0}^{\prime}\right)\right) \subseteq \operatorname{Susp}\left(\rho_{=0}, \sigma_{\text {Agt }}\left(\rho_{=0}\right)\right)$.

- Assume we have built $\rho_{\leq i}^{\prime}$ and $\sigma_{\text {Agt }}^{\prime}$ on all the prefixes of $\rho_{\leq i}^{\prime}$, and that they are such that $\rho_{\leq i} \triangleleft \rho_{\leq i}^{\prime}$ and $\operatorname{Susp}\left(\rho_{\leq i}^{\prime}, \sigma_{\text {Agt }}^{\prime}\right) \subseteq \operatorname{Susp}\left(\rho_{\leq i}, \sigma_{\text {Agt }}\right)$ (notice that $\operatorname{Susp}\left(\rho_{\leq i}^{\prime}, \sigma_{\text {Agt }}^{\prime}\right)$ only depends on the value of $\sigma_{\text {Agt }}^{\prime}$ on all the prefixes of $\left.\rho_{\leq i}\right)$. In particular, we have $\rho_{=i} \triangleleft \rho_{=i}^{\prime}$, so that with the move $\sigma_{\text {Agt }}\left(\rho_{\leq i}\right)$, we can associate a move $m_{\text {Agt }}^{\prime}$ (to which we set $\sigma_{\text {Agt }}^{\prime}\left(\rho_{\leq i}^{\prime}\right)$ ) satisfying both conditions of Definition 5.22. This defines $\rho_{=i+1}^{\prime}$ in such a way that $\rho_{\leq i+1} \triangleleft \rho_{\leq i+1}^{\prime} ; \operatorname{moreover}, \operatorname{Susp}\left(\rho_{\leq i+1}^{\prime}, \sigma_{\text {Agt }}^{\prime}\right)=\operatorname{Susp}\left(\rho_{\leq i}^{\prime}, \sigma_{\text {Agt }}^{\prime}\right) \cap \operatorname{Susp}\left(\left(\rho_{=i}^{\prime}, \rho_{=i+1}^{\prime}\right), m_{\text {Agt }}^{\prime}\right)$ is indeed a subset of $\operatorname{Susp}\left(\rho_{\leq i+1}, \sigma_{\mathrm{Agt}}\right)$.

It remains to define $\sigma_{\text {Agt }}^{\prime}$ outside its outcome $\rho^{\prime}$. Notice that, for our purposes, it suffices to define $\sigma_{\text {Agt }}^{\prime}$ on histories starting from $s_{0}^{\prime}$. We again proceed by induction on the length of the histories, defining $\sigma_{\text {Agt }}^{\prime}$ in order to satisfy (a) on prefixes of plays of $\mathcal{G}^{\prime}$ from $s_{0}^{\prime}$. At each step, we also make sure that for every $h^{\prime} \in \operatorname{Hist}_{\mathcal{G}^{\prime}}\left(s_{0}^{\prime}\right)$, there exists $h \in \operatorname{Hist}_{\mathcal{G}}(s)$ such that $h \triangleleft h^{\prime}, \operatorname{Susp}\left(h^{\prime}, \sigma_{\text {Agt }}^{\prime}\right) \subseteq \operatorname{Susp}\left(h, \sigma_{\text {Agt }}\right)$, and $\sigma_{\text {Agt }}(h)$ and $\sigma_{\text {Agt }}^{\prime}\left(h^{\prime}\right)$ satisfy the conditions of Definition 5.22 in the last states of $h$ and $h^{\prime}$, resp.

As we only consider histories from $s_{0}^{\prime}$, the case of histories of length zero was already handled. Assume we have defined $\sigma_{\text {Agt }}^{\prime}$ for histories $h^{\prime}$ of length $i$, and fix a new history $h^{\prime} \cdot t^{\prime} \in \operatorname{Hist}_{\mathcal{G}^{\prime}}\left(s_{0}^{\prime}\right)$ of length $i+1$ (that is not a prefix of $\rho$ ). By induction hypothesis, there is $h \in \operatorname{Hist}_{\mathcal{G}}\left(s_{0}\right)$ such that $h \triangleleft h^{\prime}$, and $\operatorname{Susp}\left(h^{\prime}, \sigma_{\mathrm{Agt}}^{\prime}\right) \subseteq \operatorname{Susp}\left(h, \sigma_{\mathrm{Agt}}\right)$, and $\sigma_{\mathrm{Agt}}(h)$ and $\sigma_{\mathrm{Agt}}\left(h^{\prime}\right)$ satisfy the required properties. In particular, with $t^{\prime}$, we can associate $t$ s.t. $t \triangleleft t^{\prime}$ and $\operatorname{Susp}\left(\left(\operatorname{last}\left(h^{\prime}\right), t^{\prime}\right), \sigma_{\text {Agt }}^{\prime}\left(h^{\prime}\right)\right) \subseteq \operatorname{Susp}\left((\operatorname{last}(h), t), \sigma_{\text {Agt }}(h)\right)$. Then $(h \cdot t) \triangleleft\left(h^{\prime} \cdot t^{\prime}\right)$. Since $t \triangleleft t^{\prime}$, there is a move $m_{\text {Agt }}^{\prime}$ associated with $\sigma_{\text {Agt }}(h \cdot t)$ and satisfying the conditions of Definition 5.22, Letting $\sigma_{\text {Agt }}^{\prime}\left(h^{\prime} \cdot t^{\prime}\right)=m_{\text {Agt }}^{\prime}$, we fulfill all the requirements of our induction hypothesis. 
We now need to lift the property from histories to infinite paths. Consider a play $\bar{\rho}^{\prime} \in \operatorname{Play}_{\mathcal{G}^{\prime}}\left(s_{0}^{\prime}\right)$, we will construct a corresponding play $\bar{\rho}$ in $\mathcal{G}$. Set $\bar{\rho}_{0}=s_{0}$. If $\bar{\rho}$ has been defined up to index $i$ and $\bar{\rho}_{i} \triangleleft \bar{\rho}_{i}^{\prime}$ (this is true for $i=0$ ), thanks to the way $\sigma_{\text {Agt }}^{\prime}$ is constructed, $\sigma_{\text {Agt }}\left(\bar{\rho}_{\leq i}\right)$ and $\sigma_{\text {Agt }}^{\prime}\left(\bar{\rho}_{\leq i}^{\prime}\right)$ satisfy the conditions of Definition 5.22 in $\bar{\rho}_{\leq i}$ and $\bar{\rho}_{i}^{\prime}$, respectively. We then pick $\bar{\rho}_{i+1}$ such that $\bar{\rho}_{i+1} \triangleleft \bar{\rho}_{i+1}^{\prime}$ and $\operatorname{Susp}\left(\left(\bar{\rho}_{i}, \bar{\rho}_{i+1}\right), \sigma_{\text {Agt }}\left(\bar{\rho}_{i}\right)\right) \subseteq$ $\operatorname{Susp}\left(\left(\bar{\rho}_{i}^{\prime}, \bar{\rho}_{i+1}^{\prime}\right), \sigma_{\mathrm{Agt}}^{\prime}\left(\bar{\rho}_{i}^{\prime}\right)\right)$. This being true at each step, the path $\bar{\rho}$ that is obtained, is such that $\bar{\rho} \triangleleft \bar{\rho}^{\prime}$ and $\operatorname{Susp}\left(\bar{\rho}^{\prime}, \sigma_{\text {Agt }}^{\prime}\right) \subseteq \operatorname{Susp}\left(\bar{\rho}, \sigma_{\text {Agt }}\right)$. This is the desired property.

5.7.2. Product of a game with deterministic Rabin automata. After this digression on game simulation, we come back to the game $\mathcal{G}=\left\langle\right.$ States, Agt, Act, Mov, Tab, $\left.\left(\precsim_{A}\right)_{A \in \text { Agt }}\right\rangle$, where we assume that some player $A$ has her objective given by a deterministic Rabin automaton $\mathcal{A}=\left\langle Q\right.$, States, $\left.\delta, q_{0},\left(Q_{i}, R_{i}\right)_{i \in \llbracket 1, n \rrbracket}\right\rangle$ (recall that this automaton reads sequences of states of $\mathcal{G}$, and accepts the paths that are winning for player $A$ ). We show how to compute Nash equilibria in $\mathcal{G}$ by building a product $\mathcal{G}^{\prime}$ of $\mathcal{G}$ with the automaton $\mathcal{A}$ and by computing the Nash equilibria in the resulting game, with a Rabin winning condition for $A$.

We define the product of the game $\mathcal{G}$ with the automaton $\mathcal{A}$ as the game $\mathcal{G} \ltimes \mathcal{A}=$ $\left\langle\right.$ States', Agt, Act, Mov', $\left.\operatorname{Tab}^{\prime},\left(\precsim_{A}^{\prime}\right)_{A \in \text { Agt }}\right\rangle$, where:

- States $^{\prime}=$ States $\times Q$;

- $\operatorname{Mov}^{\prime}\left((s, q), A_{j}\right)=\operatorname{Mov}\left(s, A_{j}\right)$ for every $A_{j} \in \operatorname{Agt}$;

- $\operatorname{Tab}^{\prime}\left((s, q), m_{\mathrm{Agt}}\right)=\left(s^{\prime}, q^{\prime}\right)$ where $\operatorname{Tab}\left(s, m_{\mathrm{Agt}}\right)=s^{\prime}$ and $\delta(q, s)=q^{\prime}$;

- If $B=A$ then $\precsim_{B}^{\prime}$ is given by the internal Rabin condition $Q_{i}^{\prime}=$ States $\times Q_{i}$ and $R_{i}^{\prime}=$ States $\times R_{i}^{\prime}$. Otherwise $\precsim_{B}^{\prime}$ is derived from $\precsim_{B}$, defined by $\rho \precsim_{B}^{\prime} \bar{\rho}$ if, and only if, $\operatorname{proj}(\rho) \precsim_{B} \operatorname{proj}(\bar{\rho})$ (where $\operatorname{proj}$ is the projection of States' on States). Notice that if $\precsim_{B}$ is an internal Rabin condition, then so is $\precsim_{B}^{\prime}$.

Lemma 5.24. $\mathcal{G} \ltimes \mathcal{A}$ game-simulates $\mathcal{G}$, with game simulation defined according to the projection: $s \triangleleft\left(s^{\prime}, q\right)$ iff $s=s^{\prime}$. This game simulation is preference-preserving.

Conversely, $\mathcal{G}$ game-simulates $\mathcal{G} \ltimes \mathcal{A}$, with game simulation defined by $(s, q) \triangleleft^{\prime} s^{\prime}$ iff $s=s^{\prime}$, which is also preference-preserving.

Proof. We begin with proving that both relations are preference-preserving. First notice that if $\left(\left(s_{n}, q_{n}\right)\right)_{n \geq 0}$ is a play in $\mathcal{G} \ltimes \mathcal{A}$, then its proj-projection $\left(s_{n}\right)_{n \geq 0}$ is a play in $\mathcal{G}$. Conversely, if $\rho=\left(s_{n}\right)_{n \geq 0}$ is a play in $\mathcal{G}$, then there is a unique path $\left(q_{n}\right)_{n \geq 0}$ from initial state $q_{0}$ in $\mathcal{A}$ which reads it, and $\left(\left(s_{n}, q_{n}\right)\right)_{n \geq 0}$ is then a path in $\mathcal{G} \ltimes \mathcal{A}$ that we write $\operatorname{proj}^{-1}(\rho)=\left(\left(s_{n}, q_{n}\right)\right)_{n \geq 0}$. That way, $\operatorname{proj}$ defines a one-to-one correspondence between plays in $\mathcal{G}$ and plays in $\mathcal{G} \ltimes \mathcal{A}$ where the second component starts in $q_{0}$. For a player $B \neq A$, the objective is defined so that $\operatorname{proj}(\rho)$ has the same payoff as $\rho$. Consider now player $A$, she is winning in $\mathcal{G}$ for $\rho=\left(s_{n}\right)_{n \geq 0}$ iff $\left(s_{n}\right)_{n \geq 0} \in \mathcal{L}(\mathcal{A})$ iff the unique path $\left(q_{n}\right)_{n \geq 0}$ from initial state $q_{0}$ that reads $\left(s_{n}\right)_{n \geq 0}$ satisfies the Rabin condition $\left(Q_{i}, R_{i}\right)_{i \in \llbracket 1, n \rrbracket}$ in $\mathcal{A}$ iff $\operatorname{proj}^{-1}(\rho)$ satisfies the internal Rabin condition $\left(Q_{i}^{\prime}, R_{i}^{\prime}\right)_{i \in \llbracket 1, n \rrbracket}$ in $\mathcal{G} \ltimes \mathcal{A}$. This proves that $\triangleleft$ is winning-preserving.

It remains to show that both relations are game simulations. Assume $s \triangleleft(s, q)$ and pick a move $m_{\text {Agt }}$ in $\mathcal{G}$. It is also a move in $\mathcal{G} \ltimes \mathcal{A}$, and $\operatorname{Tab}^{\prime}\left((s, q), m_{\text {Agt }}\right)=\left(\operatorname{Tab}\left(s, m_{\text {Agt }}\right), \delta(q, s)\right)$. By definition of $\triangleleft$ it then holds that $\operatorname{Tab}\left(s, m_{\text {Agt }}\right) \triangleleft \operatorname{Tab}^{\prime}\left((s, q), m_{\text {Agt }}\right)$, which proves condition (11) of the definition of a game simulation. It remains to show condition (2). Pick a state $\left(s^{\prime}, q^{\prime}\right) \in$ States $^{\prime}$. We distinguish two cases 
- If $\delta(q, s) \neq q^{\prime}$ then $\operatorname{Susp}\left(\left((s, q),\left(s^{\prime}, q^{\prime}\right)\right), m_{\mathrm{Agt}}\right)=\varnothing$, and condition (2) trivially holds.

- Otherwise $\delta(q, s)=q^{\prime}$. In that case, for any move $m_{\text {Agt }}^{\prime}$, we have that $\operatorname{Tab}\left(s, m_{\text {Agt }}^{\prime}\right)=s^{\prime}$ if, and only if, $\operatorname{Tab}^{\prime}\left((s, q), m_{\text {Agt }}^{\prime}\right)=\left(s^{\prime}, q^{\prime}\right)$. It follows that $\operatorname{Susp}\left(\left((s, q),\left(s^{\prime}, q^{\prime}\right)\right), m_{\text {Agt }}\right)=$ $\operatorname{Susp}\left(\left(s, s^{\prime}\right), m_{\text {Agt }}\right)$, which implies condition (2).

This proves that $\mathcal{G} \ltimes \mathcal{A}$ game-simulates $\mathcal{G}$.

We now assume $(s, q) \triangleleft^{\prime} s$ and pick a move $m_{\mathrm{Agt}}$ in $\mathcal{G} \ltimes \mathcal{A}$. It is also a move in $\mathcal{G}$, and as previously, condition (11) obviously holds. Pick now $s^{\prime} \in$ States. We define $q^{\prime}=$ $\delta(q, s)$, and we have $\left(s^{\prime}, q^{\prime}\right) \triangleleft s^{\prime}$ by definition of $\triangleleft^{\prime}$. As before, we get condition (2) because $\operatorname{Susp}\left(\left((s, q),\left(s^{\prime}, q^{\prime}\right)\right), m_{\text {Agt }}\right)=\operatorname{Susp}\left(\left(s, s^{\prime}\right), m_{\text {Agt }}\right)$.

We will solve the case where each player's objective is given by a deterministic Rabin automaton by applying the above result inductively. We will obtain a game where each player has an internal Rabin winning condition. Applying Proposition 5.23 each time, we get the following result:

Proposition 5.25. Let $\mathcal{G}=\left\langle\right.$ States, Agt, Act, Mov, Tab, $\left.\left(\precsim_{A}\right)_{A \in \text { Agt }}\right\rangle$ be a finite concurrent game, where for each player $A$, the preference relation $\precsim_{A}$ is single-objective given by a deterministic Rabin automaton $\mathcal{A}$. Write Agt $=\left\{A_{1}, \ldots, A_{n}\right\}$. There is a Nash equilibrium $\sigma_{\text {Agt }}$ in $\mathcal{G}$ from some state $s$ with outcome $\rho$ iff there is a Nash equilibrium $\sigma_{\text {Agt }}^{\prime}$ in $\mathcal{G}^{\prime}=$ $\left(\left(\left(\mathcal{G} \ltimes \mathcal{A}_{1}\right) \ltimes \mathcal{A}_{2}\right) \cdots \times \mathcal{A}_{n}\right)$ from $\left(s, q_{01}, \ldots, q_{0 n}\right)$ with outcome $\rho^{\prime}$, where $q_{0 i}$ is the initial state of $\mathcal{A}_{i}$ and $\rho$ is the projection of $\rho^{\prime}$ on $\mathcal{G}$.

5.7.3. Algorithm. Assume that the objective of player $A_{i}$ is given by a deterministic Rabin automaton $\mathcal{A}_{i}$. The algorithm for solving the constrained NE existence problem starts by computing the product of the game with the automata: $\mathcal{G}^{\prime}=\left(\left(\left(\mathcal{G} \ltimes \mathcal{A}_{1}\right) \ltimes \mathcal{A}_{2}\right) \cdots \times \mathcal{A}_{n}\right)$. The resulting game has size $|\mathcal{G}| \times \prod_{j \in \llbracket 1, n \rrbracket}\left|\mathcal{A}_{j}\right|$, which is exponential in the number of players. For each player $A_{j}(1 \leq j \leq n)$, the number of Rabin pairs in the product game is that of the original specification $\mathcal{A}_{j}$, say $k_{j}$. We then apply the deterministic algorithm that we have designed for Rabin objectives (see Subsection 5.6.3 page 39), which yields an exponential-time algorithm in our framework.

5.7.4. Hardness. We prove PSPACE-hardness in the restricted case of deterministic Büchi automata, by a reduction from (the complement of) the problem of the emptiness of the intersection of several language given by deterministic finite automata. This problem is known to be PSPACE-complete [29, Lemma 3.2.3].

We fix finite automata $\mathcal{A}_{1}, \ldots, \mathcal{A}_{n}$ over alphabet $\Sigma$. Let $\Sigma^{\prime}=\Sigma \cup\{$ init, final $\}$, where init and final are two special symbols not in $\Sigma$. For every $j \in \llbracket 1, n \rrbracket$, we construct a Büchi automaton $\mathcal{A}_{j}^{\prime}$ from $\mathcal{A}_{j}$ as follows. We add a state $F$ with a self-loop labelled by final and an initial state $I$ with a transition labelled by init to the original initial state. We add transitions labelled by final from every terminal state to $F$. We set the Büchi condition to $\{F\}$. If $\mathcal{L}_{j}$ is the language recognised by $\mathcal{A}_{j}$, then the language recognised by the Büchi automaton $\mathcal{A}_{j}^{\prime}$ is $\mathcal{L}_{j}^{\prime}=$ init $\cdot \mathcal{L}_{j} \cdot$ final ${ }^{\omega}$. The intersection of the languages recognised by the automata $\mathcal{A}_{j}$ is empty if, and only if, the intersection of the languages recognised by the automata $\mathcal{A}_{j}^{\prime}$ is empty.

We construct the game $\mathcal{G}$, with States $=\Sigma^{\prime}$. For each $j \in \llbracket 1, n \rrbracket$, there is a player $A_{j}$ whose objective is given by $\mathcal{A}_{j}^{\prime}$ and one special player $A_{0}$ whose objective is $\operatorname{States}^{\omega}$ (she is always winning). Player $A_{0}$ controls all the states and there are transitions from any state 
to the states of $\Sigma \cup\{$ final $\}$. Formally Act $=\Sigma \cup\{$ final $\} \cup \perp$, for all state $s \in$ States, $\operatorname{Mov}\left(s, A_{0}\right)=$ Act, and if $j \neq 0$ then $\operatorname{Mov}\left(s, A_{j}\right)=\{\perp\}$ and for all $\alpha \in \Sigma \cup\{$ final $\}$, $\operatorname{Tab}(s,(\alpha, \perp, \ldots, \perp))=\alpha$.

Lemma 5.26. There is a Nash equilibrium in game $\mathcal{G}$ from init where every player wins if, and only if, the intersection of the languages recognised by the automata $\mathcal{A}_{j}^{\prime}$ is not empty.

Proof. If there is such a Nash equilibrium, let $\rho$ be its outcome. The path $\rho$ forms a word of $\Sigma^{\prime}$, it is accepted by every automata $\mathcal{A}_{j}^{\prime}$ since every player wins. Hence the intersection of the languages $\mathcal{L}_{j}$ is not empty.

Conversely, if a word $w=i n i t \cdot w_{1} \cdot w_{2} \cdots$ is accepted by all the automata, player $A_{0}$ can play in a way such that everybody is winning: if at each step $j$ she plays $w_{j}$, then the outcome is $w$ which is accepted by all the automata. It is a Nash equilibrium since $A_{0}$ controls everything and cannot improve her payoff.

Since PSPACE is stable by complementation, this proves that the constrained NE existence problem is PSPACE-hard for objectives described by Büchi automata.

In order to prove hardness for the $\mathrm{NE}$ existence problem we use results from Section 3.5. Winning conditions in $E\left(E\left(\ldots\left(E\left(\mathcal{G}, A_{n}, A_{0}, \rho_{n}\right), \ldots, A_{2}, A_{0}, \rho_{2}\right), A_{1}, A_{0}, \rho_{1}\right)\right.$, where $\rho_{j}$ is a winning play for $A_{i}$, can be defined by slightly modifying automata $\mathcal{A}_{1}^{\prime}, \ldots, \mathcal{A}_{n}^{\prime}$ to take into account the new states. By Proposition 3.5, there exists a Nash equilibrium in this game if, and only, if there is one in $\mathcal{G}$ where all the players win. Hence PSPACE-hardness also holds for the NE existence problem.

\section{ORdered BüChI obJeCtives}

In this Section we assume that preference relations of the players are given by ordered Büchi objectives (as defined in Section 2.5), and we prove the results listed in Table 2 (page 3). We first consider the general case of preorders given as Boolean circuits, and then exhibit several simpler cases.

For the rest of this section, we fix a game $\mathcal{G}=\left\langle\right.$ States, Agt, Act, Mov, Tab, $\left.\left(\precsim_{A}\right)_{A \in \mathrm{Agt}}\right\rangle$, and assume that $\precsim_{A}$ is given by an ordered Büchi objective $\omega_{A}=\left\langle\left(\Omega_{i}^{A}\right)_{1 \leq i \leq n_{A}},\left(\lesssim_{A}\right)_{A \in \text { Agt }}\right\rangle$.

\subsection{General case: preorders are given as circuits.}

Theorem 6.1. For finite games with ordered Büchi objectives where preorders are given as Boolean circuits, the value problem, the NE existence problem and the constrained NE existence problem are PSPACE-complete.

Proof. We explain the algorithm for the constrained NE existence problem. We assume that for each player $A$, the preorder $\lesssim_{A}$ is given by a Boolean circuit $C_{A}$. The algorithm proceeds by trying all the possible payoffs for the players.

Fix such a payoff $\left(v^{A}\right)_{A \in \mathrm{Agt}}$, with $v^{A} \in\{0,1\}^{n_{A}}$ for every player $A$. We build a circuit $D_{A}$ which represents a single objective for player $A$. Inputs to circuit $D_{A}$ will be states of the game. This circuit is constructed from $C_{A}$ as follows: We set all input gates $w_{1} \cdots w_{n}$ of circuit $C_{A}$ to the value given by payoff $v^{A}$; The former input $v_{i}$ receives the disjunction of all the states in $\Omega_{i}$; We negate the output. It is not hard to check that the new circuit $D_{A}$ is such that for every play $\rho, D_{A}[\operatorname{Inf}(\rho)]$ evaluates to true if, and only if, payoff ${ }_{A}(\rho) \mathbb{L}_{A} v^{A}$, i.e. if $\rho$ is an improvement for player $A$. 
Circuit $D_{A}$ is now viewed as a single objective for player $A$, we write $\mathcal{G}^{\prime}$ for the new game. We look for Nash equilibria in this new game, with payoff 0 for each player. Indeed, a Nash equilibrium $\sigma_{\text {Agt }}$ in $\mathcal{G}$ with payoff $\left(v^{A}\right)_{A \in \text { Agt }}$ is a Nash equilibrium in game $\mathcal{G}^{\prime}$ with payoff $(0, \ldots, 0)$. Conversely a Nash equilibrium $\sigma_{\text {Agt }}$ in game $\mathcal{G}^{\prime}$ with payoff $(0, \ldots, 0)$ is a Nash equilibrium in $\mathcal{G}$ as soon as the payoff of its outcome (in $\mathcal{G}$ ) is $\left(v^{A}\right)_{A \in \mathrm{Agt}}$.

We use the algorithm described in Section 5.5. for computing Nash equilibria with single objectives given as Boolean circuits, and we slightly modify it to take into account the constraint that it has payoff $v^{A}$ for each player $A$. This can be done in polynomial space, thanks to Proposition 3.1; it is sufficient to look for plays of the form $\pi \cdot \tau^{\omega}$ with $|\pi| \leq \mid$ States $\left.\right|^{2}$ and $|\tau| \leq \mid$ States $\left.\right|^{2}$.

PSPACE-hardness was proven for single objectives given as a Boolean circuit (the circuit evaluates by setting to true all states that are visited infinitely often, and to false all other states) in Section 5.5. This kind of objective can therefore be seen as an ordered Büchi objective with a preorder given as a Boolean circuit.

6.2. When the ordered objective can be (co-)reduced to a single Büchi objective. For some ordered objectives, the preference relation can (efficiently) be reduced to a single objective. For instance, a disjunction of several Büchi objectives can obviously be reduced to a single Büchi objective, by considering the union of the target sets. Formally, we say that an ordered Büchi objective $\omega=\left\langle\left(\Omega_{i}\right)_{1 \leq i \leq n}, \lesssim\right\rangle$ is reducible to a single Büchi objective if, given any payoff vector $v$, we can construct in polynomial time a target set $\widehat{T}(v)$ such that for all paths $\rho, v \lesssim \operatorname{payoff}_{\omega}(\rho)$ if, and only if, $\operatorname{Inf}(\rho) \cap \widehat{T}(v) \neq \varnothing$. It means that securing payoff $v$ corresponds to ensuring infinitely many visits to the new target set. Similarly, we say that $\omega$ is co-reducible to a single Büchi objective if for any vector $v$ we can construct in polynomial time a target set $\widehat{T}(v)$ such that $\operatorname{payoff}_{\omega}(\rho) \not{Z} v$ if, and only if, $\operatorname{Inf}(\rho) \cap \widehat{T}(v) \neq \varnothing$. It means that improving on payoff $v$ corresponds to ensuring infinitely many visits to the new target

We prove the following proposition, which exploits (co-)reducibility for efficiently solving the various problems.

\section{Proposition 6.2.}

- For finite games with ordered Büchi objectives which are reducible to single Büchi objectives, and in which the preorders are non-trivia 6 and monotonic, the value problem is $\mathrm{P}$-complete.

- For finite games with ordered Büchi objectives which are co-reducible to single Büchi objectives, and in which the preorders are non-trivial and monotonic the NE existence problem and the constrained $N E$ existence problem are $\mathrm{P}$-complete.

Note that the hardness results follow from the hardness of the same problems for single Büchi objectives (see Section 5.3). We now prove the two upper bounds.

6.2.1. Reducibility to single Büchi objectives and the value problem. We transform the ordered Büchi objectives of the considered player into a single Büchi objective, and use a polynomial-time algorithm [23, Chapter 2] to solve the resulting zero-sum (turn-based) Büchi game.

\footnotetext{
${ }^{6}$ That is, there is more than one class in the preorder.
} 
6.2.2. Co-reducibility to single Büchi objectives and the (constrained) NE existence problem. We assume that the ordered objectives $\left(\omega_{A}\right)_{A \in \text { Agt }}$ are all co-reducible to single Büchi objectives. We show that we can use the algorithm presented in Section 5.3.2 to solve the constrained NE existence problem in polynomial time.

We first notice that the preference relations $\precsim_{A}$ satisfy the hypotheses $(\star)$ (see page 30): $(\star)_{a}$ and $(\star)_{b}$ are obvious, and $(\star)_{c}$ is by co-reducibility of the ordered objectives. It means that we can apply the results of Lemmas 5.10 and 5.11 to the current framework. To be able to conclude and apply Lemma 5.12, we need to show that for every payoff $v$, we can compute in polynomial time the set $W(\mathcal{G}, v)$ in the suspect game $\mathcal{H}(\mathcal{G}, v)$.

Lemma 6.3. Fix a threshold $v$. The set $W(\mathcal{G}, v)$ can be computed in polynomial time.

Proof. As the ordered objectives are co-reducible to single Büchi objectives, we can construct in polynomial time target sets $\widehat{T}^{A}(v)$ for each player $A$. The objective of Eve in the suspect game $\mathcal{H}(\mathcal{G}, K)$ is then equivalent to a co-Büchi objective with target set $\left\{\left(\widehat{T}^{A}(v, P) \mid A \in P\right\}\right.$. The winning region $W(\mathcal{G}, v)$ can then be determined using a polynomial time algorithm of [23, Sect. 2.5.3].

6.2.3. Applications. We will give preorders to which the above applies, allowing to infer several P-completeness results in Table 2 (those written with reference "Section 6.2]').

We first show that reducibility and co-reducibility coincide when the preorder is total.

Lemma 6.4. Let $\omega=\left\langle\left(\Omega_{i}\right)_{1 \leq i \leq n}\right.$, $\rangle$ be an ordered Büchi objective, and assume that $\lesssim i s$ total. Then, $\omega$ is reducible to a single Büchi objective if, and only if, $\omega$ is co-reducible to a single Büchi objective.

Proof. Let $u \in\{0,1\}^{n}$ be a vector. If $u$ is a maximal element, the new target set is empty, and thus satisfies the property for co-reducibility. Otherwise we pick a vector $v$ among the smallest elements that is strictly larger than $u$. Since the preorder is reducible to a single Büchi objective, there is a target set $\widehat{T}$ that is reached infinitely often whenever the payoff is greater than $v$. Since the preorder is total and by choice of $v$, we have $w \not z u v \lesssim w$. Thus the target set $\widehat{T}$ is visited infinitely often when $u$ is not larger than the payoff. Hence $\omega$ is co-reducible to a single Büchi objective.

The proof of the other direction is similar.

Lemma 6.5. Ordered Büchi objectives with disjunction or maximise preorders are reducible to single Büchi objectives. Ordered Büchi objectives with disjunction, maximise or subset preorders are co-reducible to single Büchi objectives.

Proof. Let $\omega=\left\langle\left(\Omega_{i}\right)_{1 \leq i \leq n}, \lesssim\right\rangle$ be an ordered Büchi objective. Assume $T_{i}$ is the target set for $\Omega_{i}$.

Assume $\lesssim$ is the disjunction preorder. If the payoff $v$ is different from $\mathbf{0}$ then we define $\widehat{T}(v)$ as the union of all the target sets: $\widehat{T}(v)=\bigcup_{i=1}^{n} T_{i}$. Then, for every run $\rho$,

$$
\begin{aligned}
v \lesssim \text { payoff }_{\omega}(\rho) & \Leftrightarrow \text { there is some } i \text { for which } \operatorname{Inf}(\rho) \cap T_{i} \neq \varnothing \\
& \Leftrightarrow \operatorname{Inf}(\rho) \cap \widehat{T}(v) \neq \varnothing
\end{aligned}
$$

If the payoff $v$ is $\mathbf{0}$ then we get the expected result with $\widehat{T}(v)=$ States. Disjunction being a total preorder, it is also co-reducible (from Lemma 6.4). 
We assume now that $\lesssim$ is the maximise preorder. Given a payoff $v$, consider the index $i_{0}=\max \left\{i \mid v_{i}=1\right\}$. We then define $\widehat{T}(v)$ as the union of the target sets that are above $i_{0}$ : $\widehat{T}(v)=\bigcup_{i \geq i_{0}} T_{i}$. The following four statements are then equivalent, if $\rho$ is a run:

$$
\begin{aligned}
v \lesssim \operatorname{payoff}_{\omega}(\rho) & \Leftrightarrow v \lesssim \mathbf{1}_{\left\{i \mid \operatorname{Inf}(\rho) \cap T_{i} \neq \varnothing\right\}} \\
& \Leftrightarrow i_{0} \leq \max \left\{i \mid \operatorname{Inf}(\rho) \cap T_{i} \neq \varnothing\right\} \\
& \Leftrightarrow \quad \exists i \geq i_{0} . \operatorname{Inf}(\rho) \cap T_{i} \neq \varnothing
\end{aligned}
$$

Hence $\omega$ is reducible, and also co-reducible as it is total, to a single Büchi objective.

Finally, we assume that $\lesssim$ is the subset preorder, and we show that $\omega$ is then coreducible to a single Büchi objective. Given a payoff $v$, the new target is the union of the target sets that are not reached infinitely often for that payoff: $\widehat{T}(v)=\bigcup_{\left\{i \mid v_{i}=0\right\}} T_{i}$. Then the following statements are equivalent, if $\rho$ is a run:

$$
\begin{aligned}
\operatorname{payoff}_{\omega}(\rho) \not z & \Leftrightarrow \mathbf{1}_{\left\{i \mid \operatorname{Inf}(\rho) \cap T_{i} \neq \varnothing\right\} \not{Z} u} \\
& \Leftrightarrow \exists i \cdot \operatorname{Inf}(\rho) \cap T_{i} \neq \varnothing \text { and } u_{i}=0 \\
& \Leftrightarrow \operatorname{Inf}(\rho) \cap \widehat{T}(v) \neq \varnothing
\end{aligned}
$$

As a corollary, we get the following result:

Corollary 6.6. For finite games with ordered Büchi objectives, with either the disjunction or the maximise preorder, the value problem is $\mathrm{P}$-complete. For finite games with ordered Büchi objectives, with either the disjunction, the maximise or the subset preorder, the NE existence problem and the constrained NE existence problem are $\mathrm{P}$-complete.

Remark 6.7. Note that we cannot infer P-completeness of the value problem for the subset preorder since the subset preorder is not total, and ordered objectives with subset preorder are not reducible to single Büchi objectives. Such an ordered objective is actually reducible to a generalised Büchi objective (several Büchi objectives should be satisfied).

6.3. When the ordered objective can be reduced to a deterministic Büchi automaton objective. For some ordered objectives, the preference relation can (efficiently) be reduced to the acceptance by a deterministic Büchi automaton. Formally, we say that an ordered objective $\omega=\left\langle\left(\Omega_{i}\right)_{1 \leq i \leq n}, \lesssim\right\rangle$ is reducible to a deterministic Büchi automaton whenever, given any payoff vector $u$, we can construct in polynomial time a deterministic Büchi automaton over States which accepts exactly all plays $\rho$ with $u \lesssim \operatorname{payoff}_{\omega}(\rho)$. For such preorders, we will see that the value problem can be solved efficiently by constructing the product of the deterministic Büchi automaton and the arena of the game. This construction does however not help for solving the (constrained) NE existence problems since the number of players is a parameter of the problem, and the size of the resulting game will then be exponential.

Proposition 6.8. For finite games with ordered Büchi objectives which are reducible to deterministic Büchi automata, the value problem is $\mathrm{P}$-complete.

Proof. Given the payoff $v^{A}$ for player $A$, the algorithm proceeds by constructing the automaton that recognises the plays with payoff higher than $v^{A}$. By performing the product with the game as described in Section 5.7.2, we obtain a new game, in which there is a winning strategy if, and only if, there is a strategy in the original game to ensure payoff $v^{A}$. In this 
new game, player $A$ has a single Büchi objective, so that the NE existence of a winning strategy can be decided in polynomial time.

Hardness follows from that of games with single Büchi objectives.

Applications. We now give preorders to which the above result applies, that is, which are reducible to deterministic Büchi automata objectives.

Lemma 6.9. An ordered objective where the preorder is either the conjunction, the subset or the lexicographic preorder is reducible to a deterministic Büchi automaton objective.

Proof. We first focus on the conjunction preorder. Let $\omega=\left\langle\left(\Omega_{i}\right)_{1 \leq i \leq n}, \lesssim\right\rangle$ be an ordered Büchi objective, where $\lesssim$ is the conjunction. For every $1 \leq i \leq n$, let $T_{i}$ be the target set defining the Büchi condition $\Omega_{i}$. There are only two possible payoffs: either all objectives are satisfied, or one objective is not satisfied. For the second payoff case, any play has a larger payoff: hence the trivial automaton (which accepts all plays) witnesses the property. For the first payoff case, we construct a deterministic Büchi automaton $\mathcal{B}$ as follows. There is one state for each target set, plus one accepting state: $Q=\left\{q_{0}, q_{1}, \ldots, q_{n}\right\}$; the initial state is $q_{0}$, and the unique repeated state is $q_{n}$. For all $1 \leq i \leq n$, the transitions are $q_{i-1} \stackrel{s}{\rightarrow} q_{i}$ when $s \in T_{i}$ and $q_{i-1} \stackrel{s}{\rightarrow} q_{i-1}$ otherwise. There are also transitions $q_{n} \stackrel{s}{\rightarrow} q_{0}$ for every $s \in$ States. Automaton $\mathcal{B}$ describes the plays that goes through each set $T_{i}$ infinitely often, hence witnesses the property. It can furthermore be computed in polynomial time. The construction is illustrated in Figure 21.

We now turn to the subset preorder. Let $\omega=\left\langle\left(\Omega_{i}\right)_{1 \leq i \leq n}, \lesssim\right\rangle$ be an ordered Büchi objective, where $\lesssim$ is the subset preorder. For every $1 \leq i \leq n$, let $T_{i}$ be the target set defining the Büchi condition $\Omega_{i}$. Fix a payoff $u$. A play $\rho$ is such that $u \lesssim \operatorname{payoff}_{\omega}(\rho)$ if, and only if, $\rho$ visits infinitely often all sets $T_{i}$ with $u_{i}=1$. This is then equivalent to the conjunction of all $\Omega_{i}$ 's with $u_{i}=1$. We therefore apply the previous construction for the conjunction and get the expected result.

We finish this proof with the lexicographic preorder. Let $\omega=\left\langle\left(\Omega_{i}\right)_{1 \leq i \leq n}, \lesssim\right\rangle$ be an ordered Büchi objective, where $\lesssim$ is the lexicographic preorder. For every $1 \leq i \leq n$, let $T_{i}$ be the target set defining the Büchi condition $\Omega_{i}$. Let $u \in\{0,1\}^{n}$ be a payoff vector. We construct the following deterministic Büchi automaton which recognises the runs whose payoff is greater than or equal to $u$.

In this automaton there is a state $q_{i}$ for each $i$ such that $u_{i}=1$, and a state $q_{0}$ that is both initial and repeated: $Q=\left\{q_{0}\right\} \cup\left\{q_{i} \mid u_{i}=1\right\}$. We write $I=\{0\} \cup\left\{i \mid u_{i}=1\right\}$. For every $i \in I$, we write $\operatorname{succ}(i)=\min (I \backslash\{j \mid j \leq i\})$, with the convention that $\min \varnothing=0$. The transition relation is defined as follows:

- for every $s \in$ States, there is a transition $q_{0} \stackrel{s}{\rightarrow} q_{\text {succ }(0)}$;

- for every $i \in I \backslash\{0\}$, we have the following transitions:

$-q_{i} \stackrel{T_{i}}{\rightarrow} q_{\mathrm{succ}(i)}$;

$-q_{i} \stackrel{T_{k} \backslash T_{i}}{\longrightarrow} q_{0}$ with $k<i$ and $u_{k}=0$;

$-q_{i} \stackrel{s}{\rightarrow} q_{i}$ for every $s \in$ States $\backslash\left(T_{i} \cup \bigcup_{k<i, u_{k}=0} T_{k}\right)$.

An example of the construction is given in Figure 22

We now prove correctness of this construction. Consider a path that goes from $q_{0}$ to $q_{0}$ : if the automaton is currently in state $q_{i}$, then since the last occurrence of $q_{0}$, at least one 
state for each target set $T_{j}$ with $j<i$ and $u_{j}=1$ has been visited. When $q_{0}$ is reached again, either it is because we have seen all the $T_{j}$ with $u_{j}=1$, or it is because the run visited some target $T_{i}$ with $u_{i}=0$ and all the $T_{j}$ such that $u_{j}=1$ and $j<i$; in both cases, the set of targets that have been visited between two visits to $q_{0}$ describes a payoff greater than $u$. Assume the play $\pi$ is accepted by the automaton; then there is a sequence of $q_{i}$ as above that is taken infinitely often, therefore payoff $_{\omega}(\pi)$ is greater than or equal to $u$ for the lexicographic order.

Conversely assume $v=\operatorname{payoff}_{\omega}(\pi)$ is greater than or equal to $u$, that we already read a prefix $\pi_{\leq k}$ for some $k$, and that the current state is $q_{0}$. Reading the first symbol in $\pi$ after position $k$, the run goes to the state $q_{i}$ where $i$ is the least integer such that $u_{i}=1$. Either the path visits $T_{i}$ at some point, or it visits a state in a target $T_{j}$, with $j$ smaller than $i$ and $v_{j}=0$, in which case the automaton goes back to $q_{0}$. Therefore from $q_{0}$ we can again come back to $q_{0}$ while reading the following of $\pi$, and the automaton accepts.

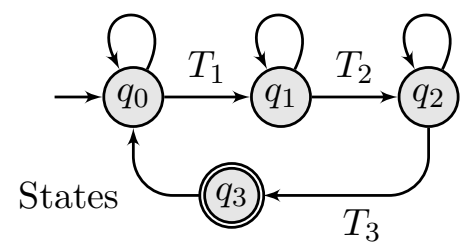

Figure 21. The automaton for the conjunction preorder, $n=3$

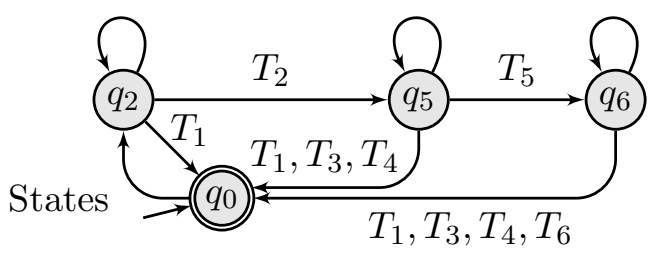

Figure 22. The automaton for the lexicographic order, $n=7$ and $u=$ $(0,1,0,0,1,1,0)$

We conclude with the following corollary:

Corollary 6.10. For finite games with ordered Büchi objectives with either of the conjunction, the lexicographic or the subset preorders, the value problem is $\mathrm{P}$-complete.

6.4. Preference relations with monotonic preorders. We will see in this part that monotonic preorders lead to more efficient algorithms. More precisely we prove the following result:

Proposition 6.11. - For finite games with ordered Büchi objectives where the preorders are given by monotonic Boolean circuits, the value problem is in coNP, and the NE existence problem and the constrained NE existence problem are in NP.

- Completeness holds in both cases for finite games with ordered Büchi objectives where the preorders are given by monotonic Boolean circuits or with the counting preorder.

- NP-completeness also holds for the constrained NE existence problem for finite games with ordered Büchi objectives where the preorders admit an element $v$ such that for every $v^{\prime}$, it holds $v^{\prime} \neq \mathbf{1} \Leftrightarrow v^{\prime} \lesssim v .7$

We first show that monotonicity of the preorders imply some memorylessness property in the suspect game. We then give algorithms witnessing the claimed upper bounds, and show the various lower bounds.

\footnotetext{
${ }^{7}$ To be fully formal, a preorder $\lesssim$ is in fact a family $\left(\lesssim_{n}\right)_{n \in \mathbb{N}}$ (where $\lesssim_{n}$ compares two vectors of size $\left.n\right)$, and this condition should be stated as "for all $n$, there is an element $v_{n} \in\{0,1\}^{n}$ such that for all $v^{\prime} \in\{0,1\}^{n}$, it holds $v^{\prime} \neq \mathbf{1} \Leftrightarrow v^{\prime} \lesssim v_{n}$ ".
} 
6.4.1. When monotonicity implies memorylessness. We say that a strategy $\sigma$ is memoryless (resp. memoryless from state $s_{0}$ ) if there exists a function $f$ : States $\rightarrow$ Act such that $\sigma(h \cdot s)=f(s)$ for every $h \in$ Hist (resp. for every $h \in \operatorname{Hist}\left(s_{0}\right)$ ). A strategy profile is said memoryless whenever all strategies of single players are memoryless. We show that when the preorders used in the ordered Büchi objectives are monotonic, the three problems are also easier than in the general case. This is because we can find memoryless trigger profiles (recall Definition 4.1).

We first show this lemma, that will then be applied to the suspect game.

Lemma 6.12. Let $\mathcal{H}$ be a turn-based two-player game. Call Eve one player, and let $\sigma_{\exists}$ be a strategy for Eve, and $s_{0}$ be a state of $\mathcal{H}$. There is a memoryless strategy $\sigma_{\exists}^{\prime}$ such that for every $\rho^{\prime} \in \operatorname{Out}_{\mathcal{H}}\left(s_{0}, \sigma_{\exists}^{\prime}\right)$, there exists $\rho \in \operatorname{Out}_{\mathcal{H}}\left(s_{0}, \sigma_{\exists}\right)$ such that $\operatorname{Inf}\left(\rho^{\prime}\right) \subseteq \operatorname{Inf}(\rho)$.

Proof. This proof is by induction on the size of the set

$$
S\left(\sigma_{1}\right)=\left\{(s, m) \mid \exists h \in \operatorname{Hist}\left(\sigma_{1}\right) . \sigma_{1}(h)=m \text { and last }(h)=s\right\} .
$$

If its size is the same as that of $\left\{s \mid \exists h \in \operatorname{Hist}\left(\sigma_{1}\right)\right.$. last $\left.(h)=s\right\}$ then the strategy is memoryless. Otherwise, let $s$ be a state at which $\sigma_{1}$ takes several different actions (i.e., $\mid(\{s\} \times$ Act $\left.) \cap S\left(\sigma_{1}\right) \mid>1\right)$.

We will define a new strategy $\sigma_{1}^{\prime}$ that takes fewer different actions in $s$ and such that for every outcome of $\sigma_{1}^{\prime}$, there is an outcome of $\sigma_{1}$ that visits (at least) the same states infinitely often.

If $\sigma$ is a strategy and $h$ is a history, we let $\sigma \circ h: h^{\prime} \mapsto \sigma\left(h \cdot h^{\prime}\right)$ for any history $h^{\prime}$. Then for every $m$ such that $(s, m) \in S\left(\sigma_{1}\right)$ we let $H_{m}=\left\{h \in \operatorname{Hist}\left(\sigma_{1}\right) \mid \operatorname{last}(h)=s\right.$ and $\left.\sigma_{1}(h)=m\right\}$, and for every $h, h^{-1} \cdot H_{m}=\left\{h^{\prime} \mid h \cdot h^{\prime} \in H_{m}\right\}$. We pick $m$ such that $H_{m}$ is not empty.

- Assume that there is $h_{0} \in \operatorname{Hist}\left(\sigma_{1}\right)$ with last $\left(h_{0}\right)=s$, such that $h_{0}^{-1} \cdot H_{m}$ is empty. We define a new strategy $\sigma_{1}^{\prime}$ as follows. If $h$ is an history which does not visit $s$, then $\sigma_{1}^{\prime}(h)=\sigma_{1}(h)$. If $h$ is an history which visits $s$, then decompose $h$ as $h^{\prime} \cdot h^{\prime \prime}$ where last $\left(h^{\prime}\right)=s$ is the first visit to $s$ and define $\sigma_{1}^{\prime}(h)=\sigma_{1}\left(h_{0} \cdot h^{\prime \prime}\right)$. Then, strategy $\sigma_{1}^{\prime}$ does not use $m$ at state $s$, and therefore at least one action has been "removed" from the strategy. More precisely, $\mid(\{s\} \times$ Act $) \cap S\left(\sigma_{1}^{\prime}\right)|\leq|(\{s\} \times$ Act $) \cap S\left(\sigma_{1}\right) \mid-1$. Furthermore the conditions on infinite states which are visited infinitely often by outcomes of $\sigma_{1}^{\prime}$ is also satisfied.

- Otherwise for any $h \in \operatorname{Hist}\left(\sigma_{1}\right)$ with last $(h)=s, h^{-1} \cdot H_{m}$ is not empty. We will construct a strategy $\sigma_{1}^{\prime}$ which plays $m$ at $s$. Let $h$ be an history, we first define the extension $e(h)$ inductively in that way:

$-e(\varepsilon)=\varepsilon$, where $\varepsilon$ is the empty history;

$-e(h \cdot s)=e(h) \cdot h^{\prime}$ where $h^{\prime} \in(e(h))^{-1} \cdot H_{m}$;

$-e\left(h \cdot s^{\prime}\right)=e(h) \cdot s^{\prime}$ if $s^{\prime} \neq s$.

We extend the definition of $e$ to infinite outcomes in the natural way: $e(\rho)_{i}=e\left(\rho_{\leq i}\right)_{i}$. We then define the strategy $\sigma_{1}^{\prime}: h \mapsto \sigma_{1}(e(h))$. We show that if $\rho$ is an outcome of $\sigma_{1}^{\prime}$, then $e(\rho)$ is an outcome of $\sigma_{1}$. Indeed assume $h$ is a finite outcome of $\sigma_{1}^{\prime}$, that $e(h)$ is an outcome of $\sigma_{1}$ and last $(h)=\operatorname{last}(e(h))$. If $h \cdot s$ is an outcome of $\sigma_{1}^{\prime}$, by construction of $e, e(h \cdot s)=e(h) \cdot h^{\prime}$, such that last $\left(h^{\prime}\right)=s$, and $h^{\prime}$ is an outcome of $\sigma_{1} \circ e(h)$ and as $e(h)$ is an outcome of $\sigma_{1}$ by hypothesis, that means that $e(h \cdot s)$ is an outcome of $\sigma_{1}$. If $h \cdot s^{\prime}$ with $s^{\prime} \neq s$ is an outcome of $\sigma_{1}^{\prime}, e\left(h \cdot s^{\prime}\right)=e(h) \cdot s^{\prime}, s^{\prime} \in \operatorname{Tab}\left(\operatorname{last}(h), \sigma_{1}^{\prime}(h)\right)$, and $\sigma_{1}^{\prime}(h)=\sigma_{1}(e(h))$. Using the hypothesis last $(h)=\operatorname{last}(e(h))$, and $e(h)$ is an outcome of $\sigma_{1}$, therefore $e\left(h \cdot s^{\prime}\right)$ is an outcome of $\sigma_{1}$. This shows that if $\rho$ is an outcome of $\sigma_{1}^{\prime}$ then 
$e(\rho)$ is an outcome of $\sigma_{1}$. The property on states visited infinitely often follows. Several moves have been removed from the strategy at $s$ (since the strategy is now memoryless at $s$, playing $m$ ).

In all cases we have $S\left(\sigma_{1}^{\prime}\right)$ strictly included in $S\left(\sigma_{1}\right)$, and an inductive reasoning entails the result.

Lemma 6.13. If for every player $A, \lesssim_{A}$ is monotonic, and if there is a trigger profile for some play $\pi$ from $s$, then there is a memoryless winning strategy for Eve in $\mathcal{H}(\mathcal{G}, \pi)$ from state ( $s, \mathrm{Agt})$.

Proof. Assume there is a trigger profile for $\pi$. We have seen in Lemma 4.4 that there is then a winning strategy $\sigma_{\exists}$ in game $\mathcal{H}(\mathcal{G}, \pi)$ for Eve. Consider the memoryless strategy $\sigma_{\exists}^{\prime}$ constructed as in Lemma 6.12, Let $\rho^{\prime}$ be an outcome of $\sigma_{\exists}^{\prime}$, there is an outcome $\rho$ of $\sigma_{\exists}$ such that $\operatorname{Inf}\left(\rho^{\prime}\right) \subseteq \operatorname{Inf}(\rho)$. As $\sigma_{\exists}$ is winning in $\mathcal{H}(\mathcal{G}, \pi)$, for every $A \in \lambda(\rho), \operatorname{proj}_{1}(\rho) \precsim A \pi$. We assume the Büchi conditions are given by the target sets $\left(T_{i}^{A}\right)_{A, i}$. For each player $A$, $\left\{i \mid \operatorname{Inf}\left(\operatorname{proj}_{1}\left(\rho^{\prime}\right)\right) \cap T_{i}^{A}\right\} \subseteq\left\{i \mid \operatorname{Inf}\left(\operatorname{proj}_{1}(\rho)\right) \cap T_{i}^{A}\right\}$. As the preorder is monotonic the payoff of $\operatorname{proj}_{1}\left(\rho^{\prime}\right)$ is smaller than that of $\operatorname{proj}_{1}(\rho): \operatorname{proj}_{1}\left(\rho^{\prime}\right) \precsim_{A} \operatorname{proj}_{1}(\rho)$. So the play is winning for any player $A$ and $\sigma_{\exists}^{\prime}$ is a memoryless winning strategy in game $\mathcal{H}(\mathcal{G}, \pi)$ for Eve.

Lemma 6.14. If for every player $A, \lesssim_{A}$ is given by monotonic Boolean circuits, then given a path $\pi$, we can decide in polynomial time if a memoryless strategy for Eve in $\mathcal{H}(\mathcal{G}, \pi)$ is winning.

Proof. Let $\sigma_{\exists}$ be a memoryless strategy in $\mathcal{H}(\mathcal{G}, \pi)$ for Eve. By keeping only the edges that are taken by $\sigma_{\exists}$, we define a subgraph of the game. We can compute in polynomial time the strongly connected components of this graph. If one component is reachable and does not satisfy the objective of Eve, then the strategy is not winning. Conversely if all the reachable strongly connected components satisfy the winning condition of Eve, since the preorder is monotonic, $\sigma_{\exists}$ is a winning strategy. Notice that since the preorder is given as a Boolean circuit, we can check in polynomial time whether a strongly connected component is winning or not. Globally the algorithm is therefore polynomial-time.

We now turn to the proof of the claimed upper bounds.

6.4.2. Proofs for the upper bounds. We show that the value problem is in coNP for finite games with ordered Büchi objectives, when preorders are given by monotonic Boolean circuits.

As already mentioned at the beginning of Section 5, for the value problem, we can make the concurrent game turn-based: since player $A$ must win against any strategy of the coalition $P=$ Agt $\backslash\{A\}$, she must also win in the case where the opponents' strategies can adapt to what $A$ plays. In other terms, we can make $A$ play first, and then the coalition. This turn-based game is determined, so that there is a strategy $\sigma$ whose outcomes are always better (for $A$ ) than $v^{A}$ if, and only if, for any strategy $\sigma^{\prime}$ of coalition $P$, there is an outcome with payoff (for $A$ ) better than $v^{A}$. If there is a counterexample to this fact, then thanks to Lemma 6.12 there is one with a memoryless strategy $\sigma^{\prime}$. The coNP algorithm proceeds by checking that all the memoryless strategies of coalition $P$ have an outcome better than $v^{A}$, which is achievable in polynomial time, with a method similar to Lemma 6.14.

We show now that the constrained NE existence problem is in NP for finite games with ordered Büchi objectives, when preorders are given by monotonic Boolean circuits. 
The algorithm for the constrained NE existence problem proceeds by guessing:

- the payoff for each player,

- a play of the form $\pi \cdot \tau^{\omega}$, where $|\pi| \leq \mid$ States $\left.\right|^{2}$ and $|\tau| \leq \mid$ States $\left.\right|^{2}$,

- an under-approximation $W$ of the set of winning states in $\mathcal{H}\left(\mathcal{G}, \pi \cdot \tau^{\omega}\right)$

- a memoryless strategy profile $\sigma_{\text {Agt }}$ in $\mathcal{H}\left(\mathcal{G}, \pi \cdot \tau^{\omega}\right)$.

We check that $\sigma_{\text {Agt }}$ is a witness for the fact that the states in $W$ are winning; thanks to Lemma 6.14, this can be done in polynomial time. We also verify that the play $\pi \cdot \tau^{\omega}$ has the expected payoff, that the payoff satisfies the constraints, and that it never gets out of $W$. If these conditions are fulfilled, then the play $\pi \cdot \tau^{\omega}$ meets the conditions of Theorem 4.5, and there is a Nash equilibrium with outcome $\pi \cdot \tau^{\omega}$. Lemma 6.13 and Proposition 3.1 ensure that if there is a Nash equilibrium, we can find it this way.

6.4.3. Proofs for the hardness results. We first prove the hardness results for the counting preorder.

Lemma 6.15. For finite games with ordered Büchi objectives that use the counting preorder, the value problem is coNP-hard.

Proof. We reduce (the complement of) 3SAT into the value problem for two-player turn-based games with Büchi objectives with the counting preorder. Consider an instance

$$
\phi=C_{1} \wedge \cdots \wedge C_{m}
$$

with $C_{j}=\ell_{j, 1} \vee \ell_{j, 2} \vee \ell_{j, 3}$, over a set of variables $\left\{x_{1}, \ldots, x_{n}\right\}$. With $\phi$, we associate a two-player turn-based game $\mathcal{G}$. Its set of states is made of

- a set containing the unique initial state $V_{0}=\left\{s_{0}\right\}$,

- a set of two states $V_{k}=\left\{x_{k}, \neg x_{k}\right\}$ for each $1 \leq k \leq n$,

- and a set of three states $V_{n+j}=\left\{t_{j, 1}, t_{j, 2}, t_{j, 3}\right\}$ for each $1 \leq j \leq m$.

Then, for each $0 \leq l \leq n+m$, there is a transition between any state of $V_{l}$ and any state of $V_{l+1}$ (assuming $V_{n+m+1}=V_{0}$ ).

The game involves two players: player $B$ owns all the states, but has no objectives (she always loses). Player $A$ has a set of Büchi objectives defined by $T_{2 \cdot k}^{A}=\left\{x_{k}\right\} \cup\left\{t_{j, p} \mid\right.$ $\left.\ell_{j, p}=x_{k}\right\}, T_{2 \cdot k+1}^{A}=\left\{\neg x_{k}\right\} \cup\left\{t_{j, p} \mid \ell_{j, p}=\neg x_{k}\right\}$, for $1 \leq k \leq n$. Notice that at least $n$ of these objectives will be visited infinitely often along any infinite play. We prove that if the formula is not satisfiable, then at least $n+1$ objectives will be fulfilled, and conversely.

Assume the formula is satisfiable, and pick a witnessing valuation $v$. We define a strategy $\sigma_{B}$ for $B$ that "follows" valuation $v$ : from states in $V_{k-1}$, for any $1 \leq k \leq n$, the strategy plays towards $x_{k}$ if $v\left(x_{k}\right)=$ true (and to $\neg x_{k}$ otherwise). Then, from a state in $V_{n+l-1}$ with $1 \leq l \leq m$, it plays towards one of the $t_{j, p}$ that evaluates to true under $v$ (the one with least index $p$, say). This way, the number of targets of player $A$ that are visited infinitely often is $n$.

Conversely, pick a play in $\mathcal{G}$ s.t. at most (hence exactly) $n$ objectives of $A$ are fulfilled. In particular, for any $1 \leq k \leq n$, this play never visits one of $x_{k}$ and $\neg x_{k}$, so that it defines a valuation $v$ over $\left\{x_{1}, \ldots, x_{n}\right\}$. Moreover, any state of $V_{n+l}$, with $1 \leq l \leq p$, that is visited infinitely often must correspond to a literal that is made true by $v$, as otherwise this would make one more objective that is fulfilled for $A$. As a consequence, each clause of $\phi$ evaluates to true under $v$, and the result follows. 


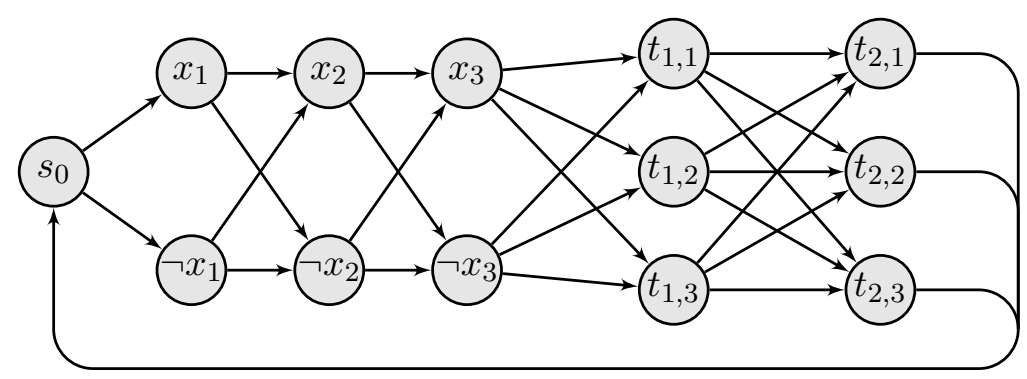

Figure 23. The game $\mathcal{G}$ associated with formula $\phi$ of 6.1

Example 6.16. We illustrate the construction of the previous proof in Figure 23 for the formula

$$
\varphi=\left(x_{1} \vee x_{2} \vee \neg x_{3}\right) \wedge\left(\neg x_{1} \vee x_{2} \vee \neg x_{3}\right) .
$$

The targets for player $A$ are $T_{1}=\left\{x_{1}, t_{1,1}\right\}, T_{2}=\left\{\neg x_{1}, t_{2,1}\right\}, T_{3}=\left\{x_{2}, t_{1,2}, t_{2,2}\right\}, T_{4}=$ $\left\{\neg x_{2}\right\}, T_{5}=\left\{x_{3}\right\}, T_{6}=\left\{\neg x_{3}, t_{1,3}, t_{2,3}\right\}$. Player $A$ cannot ensure visiting infinitely often four target sets, therefore the formula is satisfiable.

Lemma 6.17. For finite games with ordered Büchi objectives that use the counting preorder, the NE existence problem is NP-hard.

Proof. Let $\mathcal{G}$ be the game we constructed for Lemma 6.15. We construct the game $\mathcal{G}^{\prime \prime}$ from $\mathcal{G}$ as described in Section 3.3. The preference in $\mathcal{G}^{\prime}$ can still be described with ordered Büchi objectives and the counting preorder: the only target set of $B$ is $\left\{s_{1}\right\}$ and we add $s_{1}$ to $n$ different targets of $A$, where $n$ is the number of variables as in Lemma 6.15. From Proposition 3.4 there is a Nash equilibrium in $\mathcal{G}^{\prime \prime}$ from $s_{0}$ if, and only if, $A$ cannot ensure visiting at least $n+1$ targets infinitely often. Hence the NE existence problem is NP-hard.

This proves also NP-hardness for the constrained NE existence problem for ordered Büchi objectives with the counting preorder. Hardness results for preorders given by monotonic Boolean circuits follow from the above since the counting preorder is a special case of preorder given as a monotonic Boolean circuit (and the counting preorder can be expressed as a polynomial-size monotonic Boolean circuit).

We now show hardness in the special case of preorders with (roughly) at most one maximal element below 1.

Lemma 6.18. For finite turn-based games with ordered Büchi objectives with a monotonic preorder for which there is an element $v$ such that for every $v^{\prime}, v^{\prime} \neq \mathbf{1} \Leftrightarrow v^{\prime} \lesssim v$, the constrained NE existence problem is NP-hard.

Proof. Let us consider a formula $\phi=C_{1} \wedge \cdots \wedge C_{m}$ For each variable $x_{i}$, our game has one player $B_{i}$ and three states $s_{i}, x_{i}$ and $\neg x_{i}$. The objectives of $B_{i}$ are the sets $\left\{x_{i}\right\}$ and $\left\{\neg x_{i}\right\}$. Transitions go from each $s_{i}$ to $x_{i}$ and $\neg x_{i}$, and from $x_{i}$ and $\neg x_{i}$ to $s_{i+1}$ (with $s_{n+1}=s_{0}$ ). Finally, an extra player $A$ has full control of the game (i.e., she owns all the states) and has $n$ objectives, defined by $T_{i}^{A}=\left\{\ell_{i, 1}, \ell_{i, 2}, \ell_{i, 3}\right\}$ for $1 \leq i \leq n$. The construction is illustrated in Figure 24,

We show that formula $\phi$ is satisfiable if, and only if, there is a Nash equilibrium where each player $B_{i}$ gets payoff $\beta_{i}$ satisfying $\beta_{i} \lesssim v$ (hence $\beta_{i} \neq(1,1)$ ), and player $A$ gets payoff 1 . 


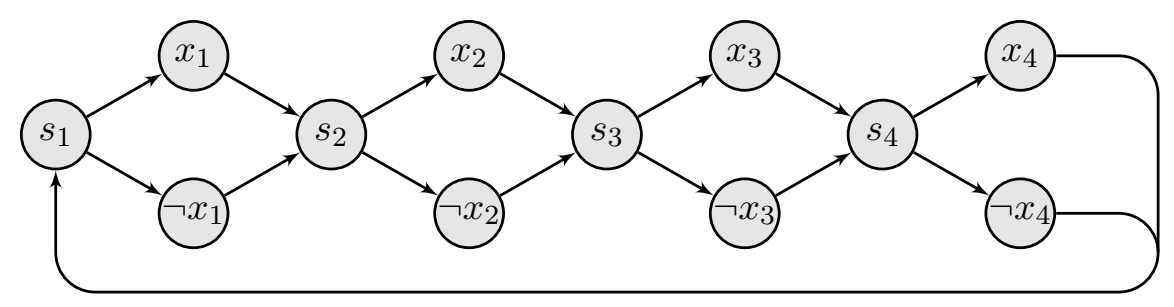

Figure 24. The Büchi game for a formula with 4 variables

First assume that the formula is satisfiable, and pick a witnessing valuation $u$. By playing according to $u$, player $A$ can satisfy all of her objectives (hence she cannot improve her payoff, since the preorder is monotonic). Since she alone controls all the game, the other players cannot improve their payoff, so that this is a Nash equilibrium. Moreover, since $A$ plays memoryless, only one of $x_{i}$ and $\neg x_{i}$ is visited for each $i$, so that the payoff $\beta_{i}$ for $B_{i}$ satisfies $\beta_{i} \lesssim v$. Conversely, if there is a Nash equilibrium with the desired payoff, then by hypothesis, exactly one of each $x_{i}$ and $\neg x_{i}$ is visited infinitely often (so that the payoff for $B_{i}$ is not $(1,1)$ ), which defines a valuation $u$. Since in this Nash equilibrium, player $A$ satisfies all its objectives, one state of each target is visited, which means that under valuation $u$, formula $\phi$ evaluates to true.

6.4.4. Applications. We now describe examples of preorders which satisfy the conditions on the existence of an element $v$ such that $v^{\prime} \neq \mathbf{1} \Leftrightarrow v^{\prime} \lesssim v$.

Lemma 6.19. Conjunction, counting and lexicographic preorders have an element $v$ such that $v^{\prime} \neq \mathbf{1} \Leftrightarrow v^{\prime} \lesssim v$.

Proof. Consider $v=(1, \ldots, 1,0)$, and $v^{\prime} \neq \mathbf{1}$. For conjunction, there is $i$ such that $v_{i}^{\prime}=0$, so $v^{\prime} \lesssim v$. For counting, $\left|\left\{i \mid v_{i}^{\prime}=1\right\}\right|<n$, so $v^{\prime} \lesssim v$. For the lexicographic preorder, let $i$ be the smallest index such that $v_{i}^{\prime}=0$, and either $v_{i}=1$ and $v_{j}=v_{j}^{\prime}$ for all $j<i$, or for all $j \in\{1, \ldots, n\}, v_{j}=v_{j}^{\prime}$. In both cases $v^{\prime} \lesssim v$.

As a consequence, the result of Lemma 6.18 applies in particular to the conjunction and lexicographic preorders, for which the constrained NE existence problem is thus NPcomplete. Hence we get:

Corollary 6.20. For finite games with ordered Büchi objectives with either of the conjunction or the lexicographic preorders, the constrained NE existence problem is NP-complete.

\section{ORDERED REACHABILITY OBJECTIVES}

In this Section we assume that preference relations of the players are given by ordered reachability objectives (as defined in Section 2.5), and we prove the results listed in Table 3 (page 3). We will first consider the general case when preorders are given by Boolean circuits and we will show that the various decision problems are PSPACE-complete. We will even notice that the hardness result holds for several simpler preorders. We will finally improve this result in a number of cases. 
For the rest of this section, we fix a game $\mathcal{G}=\left\langle\right.$ States, Agt, Act, Mov, Tab, $\left.\left(\precsim_{A}\right)_{A \in \text { Agt }}\right\rangle$, and we assume that $\precsim A$ is given by an ordered reachability objective $\omega_{A}=\left\langle\left(\Omega_{i}^{A}\right)_{1 \leq i \leq n_{A}}\right.$, $\left.\left(\lesssim_{A}\right)_{A \in \mathrm{Agt}}\right\rangle$

7.1. General case: preorders are given as circuits. We prove the following result:

\section{Proposition 7.1.}

- For finite games with ordered reachability objectives where preorders are given by Boolean circuits, the value problem, the NE existence problem and the constrained NE existence problem are in PSPACE.

- For finite two-player turn-based games with ordered reachability objectives where preorders have 1 as a unique maximal element, the value problem is PSPACE-hard.

- For finite two-player games with ordered reachability objectives where preorders have $\mathbf{1}$ as a unique maximal element, and have an element $v$ such that for every $v^{\prime}, v^{\prime} \neq \mathbf{1} \Leftrightarrow v^{\prime} \lesssim v$, then the NE existence problem and the constrained NE existence problem are PSPACE-hard.

The upper bound will be proven by reduction to games with ordered Büchi objectives using game-simulation.

7.1.1. Reduction to a game with ordered Büchi objectives. We show how to transform a game $\mathcal{G}$ with preferences given by Boolean circuits over reachability objectives into a new game $\mathcal{G}^{\prime}$, with preferences given by Boolean circuits over Büchi objectives. Although the size of $\mathcal{G}^{\prime}$ will be exponential, circuit order with Büchi objectives define prefix-independent preference relations and thus checking condition 3 of Theorem 4.5 can be made more efficient.

States of $\mathcal{G}^{\prime}$ store the set of states of $\mathcal{G}$ that have already been visited. The set of states of $\mathcal{G}^{\prime}$ is States $^{\prime}=$ States $\times 2^{\text {States }}$. The transitions are as follows: $(s, S) \rightarrow\left(s^{\prime}, S^{\prime}\right)$ when there is a transition $s \rightarrow s^{\prime}$ in $\mathcal{G}$ and $S^{\prime}=S \cup\left\{s^{\prime}\right\}$. We keep the same circuits to define the preference relations, but the reachability objectives are transformed into Büchi objectives: a target set $T$ is transformed into $T^{\prime}=\{(s, S) \mid S \cap T \neq \varnothing\}$. Although the game has exponential size, the preference relations only depend on the strongly connected components the path ends in, so that we will be able to use a special algorithm, which we describe after this lemma.

We define the relation $s \triangleleft s^{\prime}$ over states of $\mathcal{G}$ and $\mathcal{G}^{\prime}$ if, and only if, $s^{\prime}=(s, S)$ with $S \subseteq$ States, and prove that it is a game simulation (see Definition 5.22).

Lemma 7.2. The relation $\triangleleft\left(\right.$ resp. $\left.\triangleleft^{-1}\right)$ is a game simulation between $\mathcal{G}$ and $\mathcal{G}^{\prime}$, and it is preference-preserving from $\left(s_{0},\left(s_{0},\left\{s_{0}\right\}\right)\right)$ (resp. $\left.\left(\left(s_{0},\left\{s_{0}\right\}\right), s_{0}\right)\right)$.

Proof. Let $m_{\text {Agt }}$ be a move; writing $t=\operatorname{Tab}\left(s, m_{\text {Agt }}\right)$, we have $\operatorname{Tab}^{\prime}\left((s, S), m_{\text {Agt }}\right)=(t, S \cup$ $\{t\})$. Therefore $\operatorname{Tab}\left(s, m_{\mathrm{Agt}}\right) \triangleleft \operatorname{Tab}^{\prime}\left(s^{\prime}, m_{\mathrm{Agt}}\right)$. Let $\left(t, S^{\prime}\right)$ be a state of $\mathcal{G}^{\prime}$; then we also have $t \triangleleft\left(t, S^{\prime}\right)$. If $S^{\prime}=S \cup\{t\}$ then $\operatorname{Susp}\left((s, t), m_{\text {Agt }}\right)=\operatorname{Susp}\left(\left((s, S),\left(t, S^{\prime}\right)\right), m_{\text {Agt }}\right)$; otherwise $\operatorname{Susp}\left(\left((s, S),\left(t, S^{\prime}\right)\right), m_{\mathrm{Agt}}\right)=\varnothing$. In both cases, condition (2) in the definition of a game simulation is obviously satisfied.

In the other direction, let $\left(s^{\prime}, S \cup\left\{s^{\prime}\right\}\right)=\operatorname{Tab}\left((s, S), m_{\mathrm{Agt}}\right)$; we have that $s^{\prime} \triangleleft\left(s^{\prime}, S \cup\right.$ $\left.\left\{s^{\prime}\right\}\right)$. Let $t \in$ States. Then $t \triangleleft(t, S \cup\{t\})$, and $\operatorname{Susp}\left((s, t), m_{\mathrm{Agt}}\right)=\operatorname{Susp}(((s, S),(t, S \cup$ $\left.\{t\})), m_{\text {Agt }}\right)$. Hence $\triangleleft^{-1}$ is a game simulation.

Let $\rho$ and $\rho^{\prime}$ be two paths, from $s_{0}$ and $\left(s_{0},\left\{s_{0}\right\}\right)$ respectively, and such that $\rho \triangleleft \rho^{\prime}$. We show preference preservation, by showing that $\rho$ reaches target set $T$ if, and only if, 
$\rho^{\prime}$ visits $T^{\prime}$ infinitely often. If $\rho$ visits some state $s \in T$, then from that point, states visited by $\rho^{\prime}$ are of the form $\left(s^{\prime}, S^{\prime}\right)$ with $s \in S^{\prime}$; all these states are in $T^{\prime}$, therefore $\rho^{\prime}$ visits $T^{\prime}$ infinitely often. Conversely, if $\rho^{\prime}$ visits $T^{\prime}$ infinitely often, then some state of $T^{\prime}$ have been visited by $\rho$. From this, we easily obtain preference preservation.

As a corollary (Proposition 5.23) we get that there is a correspondence between Nash equilibria in $\mathcal{G}$ and Nash equilibria in $\mathcal{G}^{\prime}$.

Lemma 7.3. If there is a Nash equilibrium $\sigma_{\mathrm{Agt}}$ in $\mathcal{G}$ from $s_{0}$, then there is a Nash equilibrium $\sigma_{\text {Agt }}^{\prime}$ in $\mathcal{G}^{\prime}$ from $\left(s_{0},\left\{s_{0}\right\}\right)$ such that $\operatorname{Out}_{\mathcal{G}}\left(s_{0}, \sigma_{\text {Agt }}\right) \triangleleft \operatorname{Out}_{\mathcal{G}^{\prime}}\left(\left(s_{0},\left\{s_{0}\right\}\right), \sigma_{\text {Agt }}^{\prime}\right)$. And vice-versa: if there is a Nash equilibrium $\sigma_{\text {Agt }}^{\prime}$ in $\mathcal{G}^{\prime}$ from $\left(s_{0},\left\{s_{0}\right\}\right)$, then there is a Nash equilibrium $\sigma_{\mathrm{Agt}}$ in $\mathcal{G}$ from $s_{0}$ such that $\operatorname{Out}_{\mathcal{G}^{\prime}}\left(\left(s_{0},\left\{s_{0}\right\}\right), \sigma_{\text {Agt }}^{\prime}\right) \triangleleft^{-1} \operatorname{Out}_{\mathcal{G}}\left(s_{0}, \sigma_{\text {Agt }}\right)$.

Note that, if $\operatorname{Out}_{\mathcal{G}}\left(s_{0}, \sigma_{\mathrm{Agt}}\right) \triangleleft \operatorname{Out}_{\mathcal{G}^{\prime}}\left(\left(s_{0},\left\{s_{0}\right\}\right), \sigma_{\mathrm{Agt}}^{\prime}\right)$, then $\operatorname{Out}_{\mathcal{G}}\left(s_{0}, \sigma_{\mathrm{Agt}}\right)$ satisfies the reachability objective with target set $T$ if, and only if, $\operatorname{Out}_{\mathcal{G}^{\prime}}\left(\left(s_{0},\left\{s_{0}\right\}\right), \sigma_{\text {Agt }}^{\prime}\right)$ satisfies the Büchi objective with target set $T^{\prime}=\{(s, S) \mid S \cap T \neq \varnothing\}$. From this strong correspondence between $\mathcal{G}$ and $\mathcal{G}^{\prime}$, we get that it is sufficient to look for Nash equilibria in game $\mathcal{G}^{\prime}$.

7.1.2. How to efficiently solve the suspect game of $\mathcal{G}^{\prime}$. In game $\mathcal{G}^{\prime}$, preference relations are prefix-independent. Applying Remark 4.6 the preference relation in the suspect game is then also prefix-independent, and the payoff of a play only depends on which stronglyconnected component the path ends in. We now give an alternating algorithm which runs in polynomial time and solves the game $\mathcal{H}\left(\mathcal{G}^{\prime}, \pi^{\prime}\right)$, where $\pi^{\prime}$ is an infinite path in $\mathcal{G}^{\prime}$.

Lemma 7.4. The winner of $\mathcal{H}\left(\mathcal{G}^{\prime}, \pi^{\prime}\right)$ can be decided by an alternating algorithm which runs in time polynomial in the size of $\mathcal{G}$.

Proof. Let $C^{A}$ be the circuit defining the preference relation of player $A$. Let $\rho=\left(s_{i}, S_{i}\right)_{i \geq 0}$ be a path in $\mathcal{G}^{\prime}$, the sequence $\left(S_{i}\right)_{i \geq 0}$ is non-decreasing and converges to a limit $S(\rho)$. We have payoff ${ }_{A}(\rho)=\mathbf{1}_{\left\{i \mid T_{A}^{i} \cap S(\rho)=\varnothing\right\}}$. Therefore the winning condition of Eve in $\mathcal{H}\left(\mathcal{G}^{\prime}, \pi^{\prime}\right)$ for a play $\rho$ only depends on the limits $\lambda(\rho)$ and $S\left(\operatorname{proj}_{1}(\rho)\right)$. It can be described as a single Büchi condition with target set $T=\left\{((s, S), P) \mid \forall A \in P . C^{A}\left[v^{A}(S), w^{A}\right]\right.$ evaluates to true $\}$ where $v^{A}(S)=\mathbf{1}_{\left\{i \mid T_{A}^{i} \cap S=\varnothing\right\}}$ and $w^{A}=$ payoff $_{A}\left(\pi^{\prime}\right)$. We now describe the algorithm.

Initially the current state is set to $\left(\left(s_{0},\left\{s_{0}\right\}\right)\right.$, Agt). We also keep a list of the states which have been visited, and we initialise it with Occ $\leftarrow\left\{\left(s_{0},\left\{s_{0}\right\}\right)\right.$, Agt $\}$. Then,

- if the current state is $((s, S), P)$, the algorithm existentially guesses a move $m_{\mathrm{Agt}}$ of Eve and we set $t=\left((s, S), P, m_{\mathrm{Agt}}\right)$;

- otherwise if the current state is of the form $\left((s, S), P, m_{\text {Agt }}\right)$, it universally guesses a state $s^{\prime}$ which corresponds to a move of Adam and we set $t=\left(\left(s^{\prime}, S \cup\left\{s^{\prime}\right\}\right), P \cap \operatorname{Susp}\left(\left(s, s^{\prime}\right), m_{\mathrm{Agt}}\right)\right)$.

If $t$ was already seen (that is, if $t \in$ Occ), the algorithm returns true when $t \in T$ and false when $t \notin T$, otherwise the current state is set to $t$, and we add $t$ to the list of visited states: Occ $\leftarrow$ Occ $\cup\{t\}$, and we repeat this step. Because we stop when the same state is seen, the algorithm stops after at most $\ell+1$ steps, where $\ell$ is the length of the longest acyclic path. Since the size of $S$ can only increase and the size of $P$ only decrease, we bound $\ell$ with $\mid$ States $\left.\right|^{2} \cdot \mid$ Agt $\mid$.

We now prove the correctness of the algorithm. First, $\mathcal{H}\left(\mathcal{G}^{\prime}, \pi^{\prime}\right)$ is a turn-based Büchi game, which is a special case of parity game. Parity games are known to be determined with memoryless strategies [34, 19], hence $\mathcal{H}\left(\mathcal{G}^{\prime}, \pi^{\prime}\right)$ is determined with memoryless strategies. 
If the algorithm returns true, then there exist a strategy $\sigma_{\exists}$ of Eve such that for all the strategies $\sigma_{\forall}$ of Adam, any outcome $\rho$ of $\operatorname{Out}\left(\sigma_{\exists}, \sigma_{\forall}\right)$ is such that there exist $i<j \leq \ell+1$ with $\rho_{i}=\rho_{j} \in T$ and all $\rho_{k}$ with $k<j$ are different. We extend this strategy $\sigma_{\exists}$ to a winning strategy $\sigma_{\exists}^{\prime}$ for Eve. We do so by ignoring the loops we see in the history, formally we inductively define a reduction $r$ of histories by:

- $r(\varepsilon)=\varepsilon$;

- if $((s, S), P)$ does not appear in $r(h)$ then $r(h \cdot((s, S), P))=r(h) \cdot((s, S), P)$;

- otherwise $r(h \cdot((s, S), P))=r(h)_{\leq i}$ where $i$ is the smallest index such that $r(h)_{i}=$ $((s, S), P)$.

We then define $\sigma_{\exists}^{\prime}$ for any history $h$ by $\sigma_{\exists}^{\prime}(h)=\sigma_{\exists}(r(h))$.

We show by induction that if $h$ is a history compatible with $\sigma_{\exists}^{\prime}$ from $\left(\left(s_{0},\left\{s_{0}\right\}\right)\right.$, Agt $)$ then $r(h)$ is compatible with $\sigma_{\exists}$ from $\left(\left(s_{0},\left\{s_{0}\right\}\right)\right.$, Agt $)$. It is true when $h=\left(\left(s_{0},\left\{s_{0}\right\}\right)\right.$, Agt $)$, now assuming it holds for all history of length $\leq k$, we show it for history of length $k+1$. Let $h \cdot s$ be a history of length $k+1$ compatible with $\sigma_{\exists}^{\prime}$. By hypothesis $r(h)$ is compatible with $h$ and since $\sigma_{\exists}^{\prime}(h)=\sigma_{\exists}(r(h)), r(h) \cdot s$ is compatible with $\sigma_{\exists}$. If $r(h \cdot s)=r(h) \cdot s$ then $r(h \cdot s)$ is compatible with $\sigma_{\exists}$. Otherwise $r(h \cdot s)$ is a prefix of $r(h)$ and therefore of length $\leq k$, we can apply the induction hypothesis to conclude that $r(h \cdot s)$ is compatible with $\sigma_{\exists}$.

We now show that the strategy $\sigma_{\exists}^{\prime}$ that we defined, is winning. Let $\rho$ be a possible outcome of $\sigma_{\exists}^{\prime}$, let $i<j$ be the first indexes such that $\rho_{i}, \rho_{j} \in($ States $\times S(\rho)) \times \lambda(\rho)$ and $\rho_{i}=\rho_{j}$. Because there is no repetition between $i$ and $j-1: r\left(\rho_{\leq j-1}\right)=r\left(\rho_{\leq i-1}\right) \rho_{i} \cdots \rho_{j-1}$. We have that $\sigma_{\exists}\left(r\left(\rho_{\leq i-1}\right) \rho_{i} \cdots \rho_{j-1}\right)=\sigma_{\exists}^{\prime}\left(\rho_{j-1}\right)$. From this move, $\rho_{j}$ is a possible next state, so $r\left(\rho_{\leq i-1}\right) \rho_{i} \cdots \rho_{j}$ is a possible outcome of $\sigma_{\exists}$. As $\rho_{i}=\rho_{j}$ and all other states are different, by the hypothesis on $\sigma_{\exists}$ we have that $\rho_{j} \in T$. This shows that $\rho$ ultimately loops in states of $T$ and therefore $\rho$ is a winning run for Eve.

Reciprocally, if Eve has a winning strategy, she has a memoryless one $\sigma_{\exists}$ since this is a Büchi game. We can see this strategy as an oracle for the various existential choices in the algorithm. Consider some universal choices in the algorithm, it corresponds to a strategy $\sigma_{\forall}$ for Adam. The branch corresponding to $\left(\sigma_{\exists}, \sigma_{\forall}\right)$ ends the first time we encounter a loop, we write this history $h \cdot h^{\prime}$ with last $\left(h^{\prime}\right)=\operatorname{last}(h)$. Since the strategy $\sigma_{\exists}$ is memoryless, $h \cdot h^{\prime \omega}$ is a possible outcome. Since it is winning, last $\left(h^{\prime}\right)$ is in $T$ and therefore the branch is accepting. This being true for all the branches given by the choices of $\sigma_{\exists}$, the algorithm answers true.

7.1.3. Proof of the PSPACE upper bounds in Proposition 7.1. We describe a PSPACE algorithm for solving the constrained NE existence problem. The algorithm proceeds by trying all plays $\pi$ in $\mathcal{G}$ of the form described in Proposition 3.1. This corresponds to a (unique) play $\pi^{\prime}$ in $\mathcal{G}^{\prime}$. We check that $\pi^{\prime}$ has a payoff satisfying the constraints, and that there is a path $\rho$ in $\mathcal{H}\left(\mathcal{G}^{\prime}, \pi^{\prime}\right)$, whose projection is $\pi^{\prime}$, along which Adam obeys Eve, and which stays in the winning region of Eve. This last step is done by using the algorithm of Lemma 7.4 on each state $\rho$ goes through. All these conditions are satisfied exactly when the conditions of Theorem 4.5 are satisfied, in which case there is a Nash equilibrium within the given bounds.

The PSPACE upper bound for the value problem can be inferred from Proposition 3.2 . 
7.1.4. Proof of PSPACE-hardness for the value problem. We show PSPACE-hardness of the value problem when the preorder has $\mathbf{1}$ as a unique maximal element.

We reduce QSAT to the value problem, where QSAT is the satisfiability problem for quantified Boolean formulas. For an instance of QSAT, we assume without loss of generality that the Boolean formula is a conjunction of disjunctive clauses?

Let $\phi=Q_{1} x_{1} \ldots Q_{p} x_{p} . \phi^{\prime}$, where $Q_{i} \in\{\forall, \exists\}$ and $\phi^{\prime}=c_{1} \wedge \cdots \wedge c_{n}$ with $c_{i}=\bigvee_{1 \leq j \leq 3} \ell_{i, j}$ and $\ell_{i, j} \in\left\{x_{k}, \neg x_{k} \mid 1 \leq k \leq p\right\} \cup\{\top, \perp\}$. We define a turn-based game $\mathcal{G}(\phi)$ in the following way (illustrated in Example 7.6 below). There is one state for each quantifier, one for each literal, and two additional states $\top$ and $\perp$ :

$$
\text { States }=\left\{Q_{k} \mid 1 \leq k \leq p\right\} \cup\left\{x_{k}, \neg x_{k} \mid 1 \leq k \leq p\right\} \cup\{\top, \perp\} .
$$

The game involves two players, $A$ and $B$. The states $\top$, and $\perp$, the existential-quantifier states and the literal states are all controlled by $A$, while the universal-quantifier states belong to player $B$. For all $1 \leq k \leq p$, the state corresponding to quantifier $Q_{k}$ has two outgoing transitions, going to $x_{k}$ and $\neg x_{k}$ respectively. Those two literal states only have one transition to the next quantifier state $Q_{k+1}$, or to the final state $\top$ if $k=p$. Finally, states $T$ and $\perp$ carries a self-loop (notice that $\perp$ is not reachable, while $T$ will always be visited).

Player $A$ has one target set for each clause: if $c_{i}=\bigwedge_{1 \leq j \leq 3} \ell_{i, j}$ then $T_{i}^{A}=\left\{\ell_{i, j} \mid 1 \leq\right.$ $j \leq 3\}$. The $i$-th objective $\Omega_{i}^{A}$ is to reach target set $T_{i}^{A}$. The following result is then straightforward:

Lemma 7.5. Formula $\phi$ is valid if, and only if, player $A$ has a strategy whose outcomes from state $Q_{1}$ all visit each target set $T_{i}^{A}$.

Proof. We begin with the direct implication, by induction on $p$. For the base case, $\phi=$ $Q_{1} x_{1} . \bigwedge_{i} c_{i}$ where $c_{i}$ only involves $x_{1}$ and $\neg x_{1}$. We consider two cases:

- $Q_{1}=\exists$ : since we assume $\phi$ be true, there must exist a value for $x_{1}$ which makes all clauses true. If this value is $\top$, consider the strategy $\sigma_{\top}$ of Player $A$ such that $\sigma_{\top}\left(Q_{1}\right)=x_{1}$. Then each clause $c_{i}$ must have $x_{1}$ as one of its literals, so that the objective $\Omega_{i}^{A}$ is satisfied with this strategy. The same argument applies if the value for $x_{1}$ were $\perp$.

- $Q_{1}=\forall$ : in that case, Player $A$ has only one strategy. For both $x_{1}$ and $\neg x_{1}$ all the clauses are satisfied. It follows that each clause $c_{i}$ must contain $x_{1}$ and $\neg x_{1}$, so that objective $\Omega_{i}^{A}$ is satisfied for any strategy of player $B$.

Now, assume that the result holds for all QSAT instances with at most $p-1$ quantifiers.

- if $Q_{1}=\exists$, then one of $Q_{2} x_{2} \ldots Q_{p} x_{p} \phi^{\prime}\left[x_{1} \leftarrow \top\right]$ and $Q_{2} x_{2} \ldots Q_{p} x_{p} \phi^{\prime}\left[x_{1} \leftarrow \perp\right]$ is valid. We handle the first case, the second one being symmetric. For a literal $\lambda_{k} \in\left\{x_{k}, \neg x_{k}\right\}$, we write $T_{\lambda_{k}}$ for the set of target sets $T_{i}^{A}$ such that the clause $c_{i}$ contains the literal $\lambda_{k}$.

Assume $Q_{2} x_{2} \ldots Q_{p} x_{p} \phi^{\prime}\left[x_{1} \leftarrow \top\right]$ is valid; by induction we know that there exists a strategy $\sigma^{x_{1}}$ such that all the targets in $T_{\lambda_{k}}$ are visited along any outcome from state $Q_{2}$ (because $\mathcal{G}\left(Q_{2} x_{2} \ldots Q_{p} x_{p} \phi^{\prime}\left[x_{1} \leftarrow \top\right]\right)$ is the same game as $\mathcal{G}(\phi)$, but with $Q_{2}$ as the initial state, and with the targets in $T_{x_{1}}$ containing $\{\top\}$ in place of $x_{1}$ ). We define the strategy $\sigma$ by $\sigma\left(Q_{1}\right)=x_{1}$ and $\sigma\left(Q_{1} \cdot x_{1} \cdot \rho\right)=\sigma^{x_{1}}(\rho)$. An outcome of $\sigma$ will necessarily visit $x_{1}$, hence visiting all the targets in $T_{x_{1}}$; because $\sigma$ follows $\sigma^{x_{1}}$, all the objectives not in $T_{x_{1}}$ are met as well.

\footnotetext{
${ }^{8}$ With the convention that an empty disjunction is equivalent to $\perp$.
} 
- if $Q_{1}=\forall$, then $Q_{2} x_{2} \ldots Q_{p} x_{p} \phi^{\prime}\left[x_{1} \leftarrow \mathrm{T}\right]$ is valid. Using the induction hypothesis we know that from $Q_{2}$ there is a strategy $\sigma^{x_{1}}$ that enforces a visit to all the targets in $T_{x_{1}}$. Similarly, $Q_{2} x_{2} \ldots Q_{p} x_{p} \phi^{\prime}\left[x_{1} \leftarrow \perp\right]$ is valid, and there is a strategy $\sigma^{\neg x_{1}}$ that visits all the objectives not in $T_{\neg x_{1}}$. We define a new strategy $\sigma$ as follows: $\sigma\left(Q_{1} \cdot x_{1} \cdot \rho\right)=\sigma^{x_{1}}(\rho)$ and $\sigma\left(Q_{1} \cdot \neg x_{1} \cdot \rho\right)=\sigma \neg x_{1}(\rho)$. Consider an outcome of $\sigma$ : if it visits $x_{1}$, then all the objectives in $T_{x_{1}}$ are visited, and because the path follows $\sigma^{x_{1}}$, the objectives not in $T_{x_{1}}$ are also visited. The other case is similar.

We now turn to the converse implication. Assume the formula is not valid. We prove that for any strategy $\sigma$ of player $A$, there is an outcome $\rho$ of this strategy such that some objective $\Omega_{i}^{A}$ is not satisfied. We again proceed by induction, beginning with the case where $n=1$.

- if $Q_{1}=\exists$, then both $\phi^{\prime}\left[x_{1} \leftarrow \top\right]$ and $\phi^{\prime}\left[x_{1} \leftarrow \perp\right]$ are false. This entails that one of the clauses only involves $\perp$ (no other disjunction involving $x_{1}$ and/or $\neg x_{1}$ is always false), and the corresponding reachability condition is $\perp$, which is not reachable.

- if $Q_{1}=\forall$, then one of $\phi^{\prime}\left[x_{1} \leftarrow \top\right]$ and $\phi^{\prime}\left[x_{1} \leftarrow \perp\right]$ is false. In the former case, one of the clauses $c_{i}$ contains $\neg x_{1}$, or only contains $\perp$. Then along the run $Q_{1} \cdot x_{1} \cdot \top^{\omega}$, the objective $T_{i}^{A}$ is not visited. The other case is similar.

Now, assuming that the result holds for formulas with $n-1$ quantifiers, we prove the result with $n$ quantifiers.

- if $Q_{1}=\exists$, then both $Q_{2} x_{2} \ldots Q_{p} x_{p} \phi^{\prime}\left[x_{1} \leftarrow \top\right]$ and $Q_{2} x_{2} \ldots Q_{p} x_{p} \phi^{\prime}\left[x_{1} \leftarrow \perp\right]$ are false. Ising the induction hypothesis, any run from $Q_{2}$ fails to visit some objective not in $T_{x_{1}} \cup T_{\neg x_{1}}$. Hence no strategy from $Q_{1}$ can enforce a visit to all the objectives.

- if $Q_{1}=\forall$, then one of $Q_{2} x_{2} \ldots Q_{p} x_{p} \phi^{\prime}\left[x_{1} \leftarrow \top\right]$ and $Q_{2} x_{2} \ldots Q_{p} x_{p} \phi^{\prime}\left[x_{1} \leftarrow \perp\right]$ is false. We handle the first case, the second one being symmetric. By induction hypothesis, for any strategy $\sigma$ of player $A$ in the game $\mathcal{G}\left(\phi^{\prime}\left[x_{1} \leftarrow \top\right]\right)$, one of the outcome fails to visit all the objective not in $T_{x_{1}}$. Then along the path $\rho=Q_{1} \cdot x_{1} \cdot \rho^{\prime}$, some objectives not in $T_{x_{1}}$ are not visited.

We can directly conclude from this lemma that the value of the game for $A$ is $\mathbf{1}$ (the unique maximal payoff for our preorder) if, and only if, the formula $\phi$ is valid, which proves that the former problem is PSPACE-hard.

Example 7.6. As an example of the construction, let us consider the formula

$$
\phi=\forall x_{1} . \exists x_{2} . \forall x_{3} . \exists x_{4} .\left(x_{1} \vee \neg x_{2} \vee \neg x_{3}\right) \wedge\left(x_{1} \vee x_{2} \vee x_{4}\right) \wedge\left(\neg x_{4} \vee \perp \vee \perp\right)
$$

The target sets for player $A$ are given by $T_{1}^{A}=\left\{x_{1} ; \neg x_{2} ; \neg x_{3}\right\}, T_{2}^{A}=\left\{x_{1} ; x_{2} ; x_{4}\right\}$, and $T_{3}^{A}=\left\{\neg x_{4} ; \perp\right\}$. The structure of the game is represented in Figure 25, $B$ has a strategy that falsifies one of the clauses whatever $A$ does, which means that the formula is not valid.

7.1.5. Proof of PSPACE-hardness for the (constrained) NE existence problem. We will now prove PSPACE-hardness for the NE existence problem, under the conditions specified in the statement of Proposition 7.1, using Proposition 3.4. We specify the new preference relation for the construction of Section 3.3. We give $B$ one objective, which is to reach $s_{1}\left(s_{1}\right.$ is the sink state introduced by the construction). In terms of preferences for $A$, going $s_{1}$ should be just below visiting all targets. For this we use the statement in Proposition 7.1, that there is $v$ such that for every $v^{\prime}, v^{\prime} \neq \mathbf{1} \Leftrightarrow v^{\prime} \lesssim v$, and add $s_{1}$ as a target to each $T_{i}^{A}$ such that $v_{i}=1$. This defines a preference relation equivalent to the one in the game constructed 

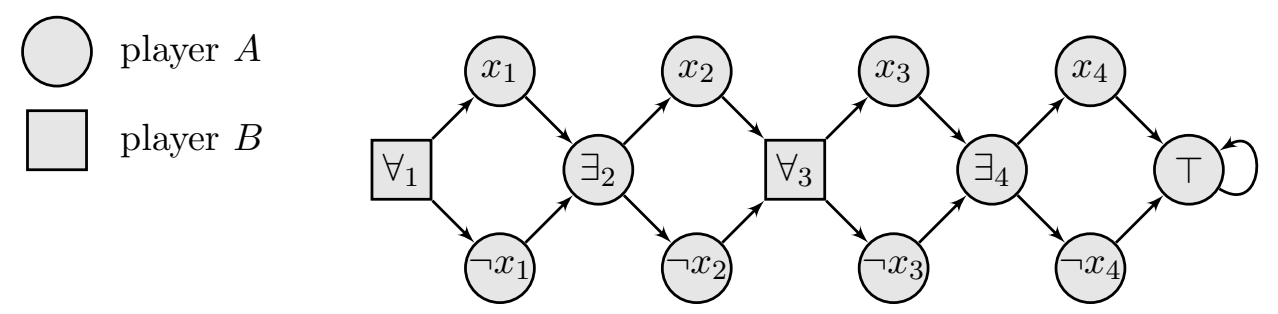

Figure 25. Reachability game associated with the formula (7.1)

in Section 3.3, therefore we deduce with Proposition 3.4 that the NE existence problem is PSPACE-hard.

7.1.6. Applications. We should now notice that conjunction, counting and lexicographic preorders (thanks to the fact that $\mathbf{1}$ is the unique maximal element for theses orders and to Lemma 6.19). As conjunction (for instance) can easily be encoded using a (monotonic) Boolean circuit in polynomial time, the hardness results are also valid if the preorder is given by a (monotonic) Boolean circuit. Finally the subset preorder can be expressed as a polynomial-size Boolean circuit and has a maximal element. We therefore get the following summary of results:

\section{Corollary 7.7.}

- For finite games with ordered reachability objectives, with either the conjunction, the counting or the lexicographic preorder, the value problem, the NE existence problem and the constrained NE existence problem are PSPACE-complete.

- For finite games with ordered reachability objectives, where the preorders are given by (monotonic) Boolean circuits, the value problem, the NE existence problem and the constrained NE existence problem are PSPACE-complete.

- For finite games with ordered reachability objectives, with the subset preorder, the value problem is PSPACE-complete.

On the other hand, the disjunction and maximise preorders do not have a unique maximal element, so the hardness result does not carry over to these preorders. In the same way, for the subset preorder, there is no $v$ such that $v^{\prime} \neq \mathbf{1} \Leftrightarrow v^{\prime} \lesssim v$, so the hardness result does not apply. We prove later (in Section 7.2) that in these special cases, the complexity is actually lower.

7.2. Simple cases. As for ordered Büchi objectives, for some ordered reachability objectives, the preference relation can be (efficiently) (co-)reduced to a single reachability objective. We do not give the formal definitions, they can easily be inferred from that for Büchi objectives on page 48 .

\section{Proposition 7.8.}

- For finite games with ordered reachability objectives which are reducible to single reachability objectives and in which the preorders are non-trivial, the value problem is $\mathrm{P}$-complete.

- For finite games with ordered reachability objectives which are co-reducible to single reachability objectives, and and in which the preorders are non-trivial, the NE existence problem and the constrained NE existence problem are NP-complete. 
Proof. Since P-hardness (resp. NP-hardness) already holds for the value (resp. NE existence) problem with a single reachability objective (see [23, Sect. 2.5.1]), we only focus on the upper bounds.

We begin with the value problem: given a payoff vector $u$ for player $A$, we build the new target set $\widehat{T}$ in polynomial time, and then use a classical algorithm for deciding whether $A$ has a winning strategy (see [23, Sect. 2.5.1]). If she does, then she can secure payoff $u$.

Consider now the constrained NE existence problem, and assume that the preference relation for each player $A$ is given by target sets $\left(T_{i}^{A}\right)_{1 \leq i \leq n_{A}}$. The NP-algorithm consists in guessing the payoff vector $\left(v_{A}\right)_{A \in \text { Agt }}$ and an ultimately periodic play $\rho=\pi \cdot \tau^{\omega}$ with $|\pi|,|\tau| \leq \mid$ States $\left.\right|^{2}$, which, for each $A$, visits $T_{i}^{A}$ if, and only if, $v_{i}^{A}=1$. We then co-reduce the payoff to a new target set $\widehat{T}^{A}\left(v^{A}\right)$ for each player $A$.

The run $\rho$ is the outcome of a Nash equilibrium with payoff $\left(v_{A}\right)_{A \in \text { Agt }}$ for the original preference relation if, and only if, $\rho$ is the outcome of a Nash equilibrium with payoff 0 with the single reachability objective $\widehat{T}^{A}\left(v^{A}\right)$ for each $A \in$ Agt. Indeed, in both cases, this is equivalent to the property that no player $A$ can enforce a payoff greater than $v^{A}$. Applying the algorithm presented in Section [5.1, this condition can be checked in polynomial time.

We now see to which ordered objectives this result applies. It is not difficult to realise that the same transformations as those made in the proof of Lemma 6.5 can be made as well for reachability objectives. We therefore get the following lemma, from which we get the remaining results in Table 3 ,

Lemma 7.9. Ordered reachability objectives with disjunction or maximise preorders are reducible to single reachability objectives. Ordered reachability objectives with disjunction, maximise or subset preorders are co-reducible to single reachability objectives.

We conclude with stating the following corollary:

\section{Corollary 7.10.}

- For finite games with ordered reachability objectives, with either the disjunction or the maximise preorder, the value problem is $\mathrm{P}$-complete.

- For finite games with ordered reachability objectives, with either the disjunction, the maximise or the subset preorder, the NE existence problem and the constrained NE existence problem are NP-complete.

\section{Conclusion}

Summary and impact of the results. Concurrent games are a natural class of games, extending classical turn-based games with more complex interactions. We have developed a complete methodology, involving new techniques, for computing pure Nash equilibria in this class of games. We were able to characterise the complexity of finding Nash equilibria (possibly with constraints on the payoff) for simple qualitative objectives first (Section [5), and then for semi-quantitative objectives (Section [6] and 7). We would like to point out that the algorithm for Büchi objectives with maximise preorder (see Section 6.2) has been implemented in tool Praline 9 [8]

\footnotetext{
${ }^{9}$ Available on http://www.lsv.ens-cachan.fr/Software/praline/
} 
We believe the methodology we have developed in this paper can be used in many other contexts, and the suspect game is a very powerful tool that will allow to analyze various properties of multi-agent systems. Indeed, the correspondence between pure Nash equilibria in the original game and winning strategies in the suspect game holds with no assumption on the structure of the game. In particular it can be applied to games given as pushdown systems, counter systems, etc. Also it does not assume anything on the preference relations, only the resulting winning condition in the suspect game can become very complex if the preference relations are complex. Now the matter is just algorithmics, in that we have to solve a two-player turn-based game in a potentially complex arena (if the original game structure is complex) with a potentially complex winning condition (if the preference relations are complex).

The suspect game construction can also be adapted to compute many other kinds of equilibria; this is for instance applied to robust equilibria in [9]. We believe this can be used in many other contexts.

We have also developed in this paper another tool that might have its own interest and be useful in some other contexts: the game-simulation (see Section [5.7.1). We used this tool several times (for handling objectives given by deterministic Rabin automata, but also for handling ordered reachability objectives). This tool can also be used to handle more complex game structures, like we did in [5] for timed games, when we originally introduced this notion. In particular, the construction done in [5] shows that we can compute Nash equilibria for timed games with all kinds of objectives studied in the current paper.

Our future researches will include extending the use of the suspect game abstraction for other families of games, and to push it further to also handle truly quantitative objectives.

Discussion on the various hypotheses made in this paper. We have assumed strategies are pure, and game structures are deterministic. This is indeed a restriction, and allowing for randomised strategies would be of great interest. Note however that pure Nash equilibria are resistant to malicious randomised players (that is, to deviations by randomised strategies). There is no obvious way to modify the suspect game construction to handle either stochastic game structures or randomised strategies. Indeed, given a history, it is hard to detect strategy deviations if they can be randomised, and therefore the set of suspects is hard to compute (and actually even define). This difficulty is non-surprising, since the existence of a Nash equilibrium in pure or randomised strategies is undecidable for stochastic games with reachability or Büchi objectives [42], and the existence of a Nash equilibrium in randomised strategies is undecidable for deterministic games [41]. However we would like to exhibit subclasses of stochastic games for which we can synthesize randomised Nash equilibria, this is part of our research programme.

We have assumed that strategies are based on histories that only record states which have been visited, and not actions which have been played. We believe this is more relevant in the context of distributed systems, where only the effect of an action might be visible to other players. Furthermore, this framework is more general than the one where every player could see the actions of the other players, since the latter can easily be encoded in the former. In the context of complete information (precise view of the actions), computing Nash equilibria is rather easy since, once a player has deviated from the equilibrium, all the other players know it and can make a coalition against that player. To illustrate that simplification, we only mention that the constrained NE existence problem falls in NP for finite games with single parity objectives (we can obtain this bound based on the suspect 
game construction), if we assume that strategies can observe actions, whereas the problem is $\mathrm{P}_{\|}^{\mathrm{NP}}$-hard if strategies do not observe the actions.

Finally we have chosen the framework of concurrent games, and not that of turn-based games as is often the case in the literature. Concurrent games naturally appear when studying timed games [5] (the semantics of a timed game is that of a concurrent game, and the abstraction based on regions that is correct for timed games is concurrent), and in

the context of distributed systems, concurrent moves are also very natural. In fact turnbased games are even a simpler case of concurrent games when we assume strategies can see the actions. Of course, the suspect game construction applies to turn-based games, but becomes quite simple (as is the case if strategies do see actions), since the set of suspect players is either the set Agt of all players (this is the case as long as no player has deviated from the equilibrium), or reduces to a singleton, as soon as a player has deviated. To illustrate this simplification, we notice that in the turn-based finite games, the constrained NE existence problem is NP-complete for single parity objectives [40] (it is $\mathrm{P}_{\|}^{\mathrm{NP}}$-complete in finite concurrent games).

Acknowledgment. We would like to thank the reviewers for their numerous comments and remarks, which helped us improve the presentation of this paper.

\section{REFERENCES}

[1] L. d. Alfaro, M. Faella, Th. A. Henzinger, R. Majumdar, and M. Stoelinga. The element of surprise in timed games. In Proc. 14th International Conference on Concurrency Theory (CONCUR'03), LNCS 2761, p. 142-156. Springer, 2003.

[2] R. Alur, Th. A. Henzinger, and O. Kupferman. Alternating-time temporal logic. J. of the ACM, 49:672$713,2002$.

[3] E. Asarin, O. Maler, A. Pnueli, and J. Sifakis. Controller synthesis for timed automata. In Proc. IFAC Symposium on System Structure and Control, p. 469-474. Elsevier Science, 1998.

[4] P. Bouyer, R. Brenguier, and N. Markey. Nash equilibria for reachability objectives in multi-player timed games. In Proc. 21th International Conference on Concurrency Theory (CONCUR'10), LNCS 6269, p. 192-206. Springer, 2010.

[5] P. Bouyer, R. Brenguier, N. Markey, and M. Ummels. Nash equilibria in concurrent games with Büchi objectives. In Proc. 30th Conference on Foundations of Software Technology and Theoretical Computer Science (FSTTCS'11), LIPIcs 13, p. 375-386. Leibniz-Zentrum für Informatik, 2011.

[6] P. Bouyer, R. Brenguier, N. Markey, and M. Ummels. Concurrent games with ordered objectives. In Proc. 15th International Conference on Foundations of Software Science and Computation Structures (FoSSaCS'12), LNCS 7253, p. 301-315. Springer, 2012.

[7] R. Brenguier. Nash Equilibria in Concurrent Games - Application to Timed Games. PhD thesis, ENS Cachan, 2012.

[8] R. Brenguier. PRALINE: A tool for computing Nash equilibria in concurrent games. In Proc. 25th International Conference on Computer Aided Verification (CAV'13), LNCS 8044, p. 890-895. Springer, 2013.

[9] R. Brenguier. Robust equilibria in concurrent games. Technical Report abs/1311.7683, CoRR, 2013. http://arxiv.org/abs/1311.7683.

[10] K. Chatterjee. Two-player nonzero-sum $\omega$-regular games. In Proc. 16th International Conference on Concurrency Theory (CONCUR'05), LNCS 3653, p. 413-427. Springer, 2005.

[11] K. Chatterjee, T. A. Henzinger, and M. Jurdziński. Games with secure equilibria. In Proc. 3rd International Symposium on Formal Methods for Components and Objects (FMCO'04), p. 141-161. Springer, 2005.

[12] K. Chatterjee, T. A. Henzinger, and N. Piterman. Generalized parity games. In Proc. 10th International Conference on Foundations of Software Science and Computation Structures (FoSSaCS'07), LNCS 4423, p. 153-167. Springer, 2007. 
[13] K. Chatterjee, T. A. Henzinger, and N. Piterman. Algorithms for Büchi games. Research Report 0805.2620, Computing Research Repository, 2008.

[14] K. Chatterjee, T. A. Henzinger, and N. Piterman. Strategy logic. Inf. \& Comp., 208(6):677 - 693, 2010. Special Issue: 18th International Conference on Concurrency Theory (CONCUR 2007).

[15] K. Chatterjee, R. Majumdar, and M. Jurdziński. On Nash equilibria in stochastic games. In Proc. 18th International Workshop on Computer Science Logic (CSL'04), LNCS 3210, p. 26-40. Springer, 2004.

[16] A. Da Costa, F. Laroussinie, and N. Markey. ATL with strategy contexts: Expressiveness and model checking. In FSTTCS'10, LIPIcs 8, p. 120-132. Leibniz-Zentrum für Informatik, 2010.

[17] J. De Pril, J. Flesch, J. Kuipers, G. Schoenmakers, and K. Vrieze. Existence of secure equilibrium in multi-player games with perfect information. In Proceedings of the 39th International Symposium on Mathematical Foundations of Computer Science (MFCS'14) Part II, LNCS 8635, p. 213-225, Budapest, Hungary, 2014. Springer.

[18] E. A. Emerson and C. S. Jutla. The complexity of tree automata and logics of programs. In Proc. 29th Annual Symposium on Foundations of Computer Science (FOCS'88), p. 328-337. IEEE Computer Society Press, 1988.

[19] E. A. Emerson and C. S. Jutla. Tree automata, mu-calculus and determinacy. In Proc. 32nd Annual Symposium on Foundations of Computer Science (FOCS'91), p. 368-377. IEEE Computer Society Press, 1991.

[20] A. M. Fink. Equilibrium in a stochastic n-person game. Journal of Science in Hiroshima University, 28(1):89-93, 1964.

[21] D. Fisman, O. Kupferman, and Y. Lustig. Rational synthesis. In Proc. 16th International Conference on Tools and Algorithms for the Construction and Analysis of Systems (TACAS'10), LNCS 6015, p. 190201. Springer, 2010.

[22] G. Gottlob. NP trees and Carnap's modal logic. J. of the ACM, 42(2):421-457, 1995.

[23] E. Grädel, W. Thomas, and Th. Wilke, editors. Automata, Logics, and Infinite Games: A Guide to Current Research, LNCS 2500. Springer, 2002.

[24] T. A. Henzinger. Games in system design and verification. In Proc. 10th Conference on Theoretical Aspects of Rationality and Knowledge (TARK'05), p. 1-4, 2005.

[25] F. Horn. Streett games on finite graphs. In Proc. 2nd Workshop on Games in Design and Verification (GDV'04), 2005.

[26] F. Horn. Explicit Muller games are PTIME. In Proc. 28th Conference on Foundations of Software Technology and Theoretical Computer Science (FSTTCS'08), LIPIcs 2, p. 235-243. Leibniz-Zentrum für Informatik, 2008.

[27] P. W. Hunter. Complexity and infinite games on finite graphs. PhD thesis, University of Cambridge, 2007.

[28] M. Jurdziński. Deciding the winner in parity games is in UP $\cap$ coUP. Information Processing Letters, 68(3):119-124, 1998.

[29] D. Kozen. Lower bounds for natural proof systems. In Proc. 18th Annual Symposium on Foundations of Computer Science (FOCS'ry), p. 254-266. IEEE Computer Society Press, 1977.

[30] F. Laroussinie, N. Markey, and G. Oreiby. On the expressiveness and complexity of ATL. Logical Methods in Computer Science, 4(2), 2008.

[31] A. B. MacKenzie and S. B. Wicker. Stability of multipacket slotted Aloha with selfish users and perfect information. In Proc. 22nd Annual Joint Conference of the IEEE Computer and Communications Societies (INFOCOM'03). IEEE Computer Society Press, 2003.

[32] R. McNaughton. Infinite games played on finite graphs. Annals of Pure and Applied Logic, 65(2):149184, 1993.

[33] F. Mogavero, A. Murano, and M. Y. Vardi. Reasoning about strategies. In Proc. 29th Conference on Foundations of Software Technology and Theoretical Computer Science (FSTTCS'10), LIPIcs 8, p. 133144. Schloss Dagstuhl - LZI, 2010.

[34] A. W. Mostowski. Games with forbidden positions. Technical Report 78, University of Gdansk, Poland, 1991.

[35] J. F. Nash. Equilibrium points in $n$-person games. Proceedings of the National Academy of Sciences of the United States of America, 36(1):48-49, 1950.

[36] Ch. H. Papadimitriou. Computational Complexity. Addison-Wesley, 1994. 
[37] C. U. Saraydar, N. B. Mandayam, and D. J. Goodman. Pareto efficiency of pricing-based power control in wireless data networks. In Proc. IEEE Wireless Communications and Networking Conference (WCNC'99), p. 231-235. IEEE Computer Society Press, 1999.

[38] L. S. Shapley. A value for n-person games. Contributions to the Theory of Games, 2:307-317, 1953.

[39] W. Thomas. Infinite games and verification. In Proc. 14th International Conference on Computer Aided Verification (CAV'02), LNCS 2404, p. 58-64. Springer, 2002. Invited Tutorial.

[40] M. Ummels. The complexity of Nash equilibria in infinite multiplayer games. In Proc. 11th International Conference on Foundations of Software Science and Computation Structures (FoSSaCS'08), LNCS 4962, p. 20-34. Springer, 2008.

[41] M. Ummels and D. Wojtczak. The complexity of Nash equilibria in limit-average games. In Proc. 22nd International Conference on Concurrency Theory (CONCUR'11), LNCS 6901, p. 482-496. Springer, 2011.

[42] M. Ummels and D. Wojtczak. The complexity of Nash equilibria in stochastic multiplayer games. Logical Methods in Computer Science, 7(3), 2011.

[43] J. von Neumann and O. Morgenstern. Theory of Games and Economic Behavior. Princeton University Press, 1944.

[44] K. W. Wagner. On restricting the access to an NP-oracle. In Proc. 15th International Colloquium on Automata, Languages and Programming (ICALP'88), LNCS 317, p. 682-696. Springer, 1988. 


\section{Appendix: Proof of Proposition 5.2}

We show PSPACE-hardness of the constrained existence of a Nash equilibrium for various kinds of qualitative objectives, using an encoding of the satisfiability of a QSAT formula $\psi=\forall x_{1} . \exists x_{2} . \ldots \forall x_{p-1} . \exists x_{p} . \bigwedge_{1 \leq i \leq n} c_{i}$, where each $c_{i}$ is of the form $\ell_{i, 1} \vee \ell_{i, 2} \vee \ell_{i, 3}$ and $\ell_{i, j} \in\left\{x_{k}, \neg x_{k} \mid 1 \leq k \leq p\right\}$.

We construct a game $\mathcal{G}_{\psi}=$ 〈States, Agt, Act, Mov, Tab, $\left.\left(\precsim_{A}\right)_{A \in \text { Agt }}\right\rangle$ as follows: States $=$ $\{u, w\} \cup \bigcup_{k \in \llbracket 1, p \rrbracket}\left\{s_{k}, t_{k}, f_{k}, d_{k}, e_{k}\right\} \cup \bigcup_{i \in \llbracket 1, n \rrbracket}\left\{b_{i}, c_{i}\right\} ;$ Agt $=\{$ Eve $\} \cup \bigcup_{k \in \llbracket 1, p \rrbracket}\left\{A_{k}, B_{k}\right\} ;$ Act $=$ $\{0,1,2\}$. We now define the transition table (the structure of the game is represented in Figure 26).

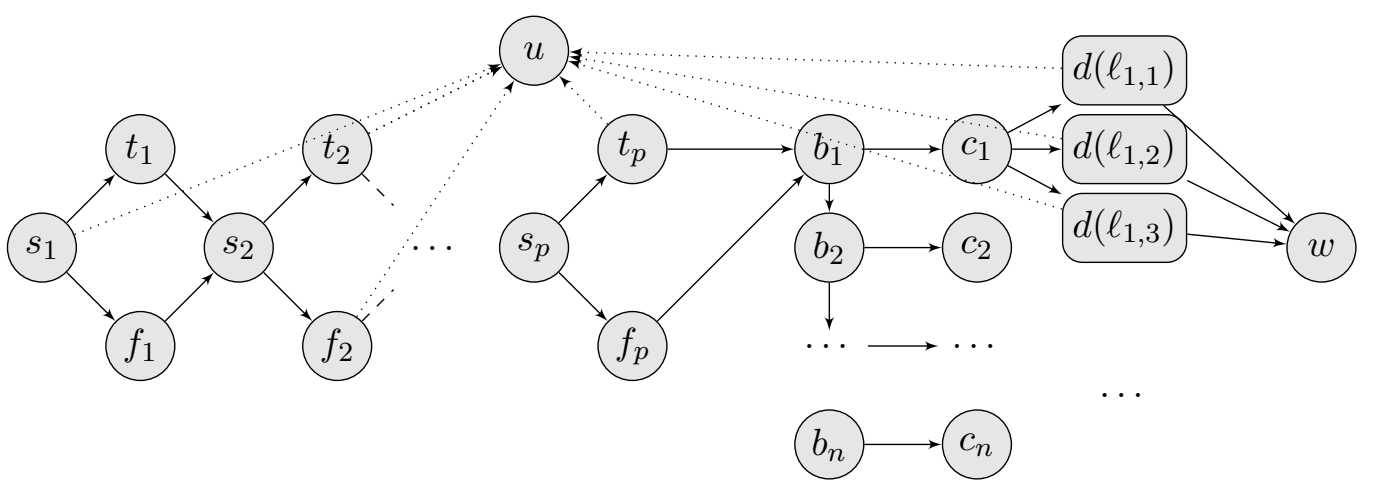

FIGURE 26. Encoding of a QSAT formula into a game with succinct representation of the transition formula. Dotted edges correspond to the strategy profile that in each states selects action 0 for every player.

- If $k \leq p$ is odd, then in state $s_{k}$, the transition function is given by 10

$$
\begin{aligned}
\left(\left(\bigotimes_{1 \leq k^{\prime} \leq p}\left(A_{k^{\prime}}=1\right) \otimes \bigotimes_{1 \leq k^{\prime} \leq p, k^{\prime} \neq k}\left(B_{k^{\prime}}=1\right), t_{k}\right),\right. \\
\left.\quad\left(\bigotimes_{1 \leq k^{\prime} \leq p, k^{\prime} \neq k}\left(A_{k^{\prime}}=0\right) \otimes \bigotimes_{1 \leq k^{\prime} \leq p}\left(B_{k^{\prime}}=0\right), f_{k}\right),(\top, u)\right)
\end{aligned}
$$

In the first part, the coalition of all the players except Eve and $B_{k}$ takes the decision to go to $t_{k}$, and any of those players can switch her action and enforce state $t_{k}$ (meaning that $x_{k}$ is set to true); if the move to state $t_{k}$ is not chosen, then the coalition of all players except Eve and $A_{k}$ takes the decision to go to $f_{k}$, and any of those players can switch her action and enforce state $f_{k}$ (meaning that $x_{k}$ is set to false); otherwise the game goes to state $u$.

In states $t_{k}$ and $f_{k}$, the transition function is $\left(T, s_{k+1}\right)$.

- If $k \leq p$ is even, then in state $s_{k}$ the transition function is given by $\left(\left(\right.\right.$ Eve $\left.\left.=1, t_{k}\right),\left(\top, f_{k}\right)\right)$ : Eve decides the value of variable $x_{k}$ (state $t_{k}$ corresponds to setting $x_{k}$ to true, and state $f_{k}$ corresponds to setting variable $x_{k}$ to false).

\footnotetext{
${ }^{10}$ The operator $\otimes$ evaluates the parity of the number of subformulas that are true: $\bigotimes_{h=1}^{g} \alpha_{h}$ is true iff $\mid\left\{\alpha_{h} \mid \alpha_{h}\right.$ evaluates to true $\} \mid$ is odd.
} 
In state $t_{k}$, the transition function is given by

$$
\left(\left(\bigvee_{1 \leq k^{\prime} \leq p}\left(A_{k^{\prime}}=1\right) \vee \bigvee_{1 \leq k^{\prime} \leq p, k^{\prime} \neq k}\left(B_{k^{\prime}}=1\right), s_{k+1}\right),(\top, u)\right)
$$

with $s_{p+1}=b_{1}$ : any player except Eve and $B_{k}$ can decide to go to state $s_{k+1}$ by playing action 1; otherwise the game proceeds to state $u$. Intuitively, any of the above players can "validate" the previous choice of Eve having set $x_{k}$ to true.

In state $f_{k}$, the transition function is given by

$$
\left(\left(\bigvee_{1 \leq k^{\prime} \leq p, k^{\prime} \neq k}\left(A_{k^{\prime}}=0\right) \vee \bigvee_{1 \leq k^{\prime} \leq p}\left(B_{k^{\prime}}=0\right), s_{k+1}\right),(\top, u)\right)
$$

with $s_{p+1}=b_{1}$ : any player except Eve and $A_{k}$ can decide to go to state $s_{k+1}$ by playing action 1; otherwise the game proceeds to state $u$. Intuitively, any of the above players can "validate" the previous choice of Eve having set $x_{k}$ to false.

- If $i \leq n$, in $b_{i}$, the transition function is given by

$$
\left(\left(\bigotimes_{1 \leq k \leq p}\left(\left(A_{k}=1\right) \otimes\left(B_{k}=1\right)\right), c_{i}\right),\left(\top, b_{i+1}\right)\right)
$$

with $b_{n+1}=u$. Intuitively the coalition of all players except Eve can decide to go to state $c_{i}$, which will mean that they want to check the truth of clause $c_{i}$. Moreover, any of those players can switch her action and decide by her own to check this clause.

- If $i \leq n$, in $c_{i}$, the transition function is given by

$$
\left(\left(\text { Eve }=1, d\left(\ell_{i, 1}\right)\right),\left(\text { Eve }=2, d\left(\ell_{i, 2}\right)\right),\left(\top, d\left(\ell_{i, 3}\right)\right)\right)
$$

where for all $1 \leq k \leq p, d\left(x_{k}\right)=d_{k}$ and $d\left(\neg x_{k}\right)=e_{k}$. Intuitively Eve proves the current clause is satisfied by pointing to the literal which is set to true.

In state $d_{k}(1 \leq k \leq p)$, the transition function is given by $\left(\left(B_{k}=1, w\right),(\top, u)\right)$. Player $B_{k}$ decides to go to $u$ or $w$.

In state $e_{k}(1 \leq k \leq p)$, the transition function is given by $\left(\left(A_{k}=1, w\right),(\top, u)\right)$.

Intuitively, in the game we have just defined, Eve will be in charge of properly choosing the value of the existentially quantified variables in $\psi$. The value of the variables will be given by the history (visiting $t_{k}$ means variable $x_{k}$ is set to true, whereas visiting $f_{k}$ means variable $x_{k}$ is set to false). Then, player $A_{k}$ will be in charge of witnessing that variable $x_{k}$ is set to true, whereas player $B_{k}$ will be in charge of witnessing that variable $x_{k}$ is set to false. Their role will be clearer in the proof.

The objective for each player $A_{k}, B_{k}$ is to reach state $w$, and for Eve to reach state $u$. This is naturally a reachability objectives but can also be encoded as a Büchi objective or a safety objective where the goal is to avoid state $u$ for $A_{k}$ and $B_{k}$, and avoid $v$ for Eve.

We will show that there is a Nash equilibrium in $\mathcal{G}_{\psi}$ where Eve wins if, and only if, $\psi$ is valid.

Before switching to the proof of this equivalence, we define a correspondence between (partial) valuations and histories in the game. with a partial valuation $v:\left\{x_{1}, \ldots, x_{k}\right\} \rightarrow$ \{true, false\}, we associate the history $\mathrm{h}(v)=s_{1} w_{1} s_{2} w_{2} \ldots w_{k} s_{k+1}$ where for all $1 \leq k^{\prime} \leq k$, $w_{k^{\prime}}=t_{k^{\prime}}\left(\right.$ resp. $\left.w_{k^{\prime}}=f_{k^{\prime}}\right)$ if $v\left(x_{k^{\prime}}\right)=$ true (resp. $v\left(x_{k^{\prime}}\right)=$ false). Conversely with every history $h$ in $\mathcal{G}_{\psi}$, we associate the partial valuation $\mathrm{v}_{h}:\left\{x_{1}, \ldots, x_{k}\right\} \rightarrow\{$ true, false $\}$ 
such that state $s_{k+1}$ (with $s_{p+1}=b_{1}$ ) is the latest such state appearing along $h$, and $v_{h}\left(x_{k^{\prime}}\right)=$ true (resp. false) if $h$ visits $t_{k^{\prime}}\left(\right.$ resp. $\left.f_{k^{\prime}}\right)$, for all $1 \leq k^{\prime} \leq k$.

Assume formula $\psi$ is valid. For all players $A_{k}$ and $B_{k}$ we set strategies $\sigma_{A_{k}}$ and $\sigma_{B_{k}}$ to always play action 2. We now turn to the strategy for Eve. Consider a history $h=$ $s_{1} \cdots w_{k-1} \cdot s_{k}$ where $k<p$ is odd. Let $v^{\prime}$ be the valuation where $v^{\prime}\left(x_{k^{\prime}}\right)=v_{h}\left(x_{k^{\prime}}\right)$ for all $k^{\prime}<$ $k$, and $v^{\prime}\left(x_{k}\right)=1$. We set $\sigma_{\text {Eve }}(h)$ to be 1 if $v^{\prime}$ makes the formula $\forall x_{k+1} \ldots \ldots \exists x_{p} . \bigwedge_{1 \leq i \leq n} c_{i}$ valid, and 0 otherwise. Since $\psi$ is valid, one of the two choices makes the rest of the formula true. This ensures that a history that reaches $b_{1}$ and that is compatible with $\sigma_{\text {Eve }}$ will define a valuation that makes $\bigwedge_{1 \leq i \leq n} c_{i}$ true. Fix a history $h$ that is compatible with $\sigma_{\text {Eve }}$ and ends up in some state $c_{i}$ : the strategy of Eve is to go to $d\left(\ell_{i, j}\right)$ where the literal $\ell_{i, j}$ makes the clause $c_{i}$ true under valuation $\mathrm{v}_{h}$. For all other histories, we set the strategy of Eve to be 2 .

We show that the strategy profile $\sigma_{\text {Agt }}=\left(\sigma_{\text {Eve }},\left(\sigma_{A_{k}}, \sigma_{B_{k}}\right)_{1 \leq k \leq p}\right)$ is a Nash equilibrium. First notice that the outcome of $\sigma_{\text {Agt }}$ is $s_{1} \cdot u$ (since all players $A_{k}$ and $B_{k}$ play action 2): Eve wins, and all the other players lose. We now describe interesting deviating strategies for the players $A_{k}$ or $B_{k}$ :

- Consider a deviating strategy $\sigma_{A_{k}}^{\prime}$ for player $A_{k}$ : let $h \in \operatorname{Out}^{\mathrm{f}}\left(\sigma_{\mathrm{Agt}}\left[A_{k} \mapsto \sigma_{A_{k}}^{\prime}\right]\right)$; if $\sigma_{A_{k}}^{\prime}(h)=2$, then $\operatorname{Out}\left(\sigma_{\mathrm{Agt}}\left[A_{k} \mapsto \sigma_{A_{k}}^{\prime}\right]\right)$ ends up in state $u$; therefore an interesting deviating strategy should choose some value 0 or 1 after any history. Now if $k^{\prime}$ is odd with $k^{\prime} \neq k$, then from $s_{k^{\prime}}$, player $A_{k}$ can choose to go to $t_{k^{\prime}}$ (action 1 ) or $f_{k^{\prime}}$ (action 0 ). If $k$ is odd, then the only way not to end up in $u$ from $s_{k}$ is to choose action 1 which leads to state $t_{k}$. Now at state $t_{k^{\prime}}$ with $k^{\prime}$ even, $\sigma_{A_{k}}^{\prime}$ should validate the choice of Eve (that is, play action 1 in $t_{k^{\prime}}$ - meaning that variable $x_{k^{\prime}}$ has value true). At state $f_{k^{\prime}}$ with $k^{\prime}$ even, if $k^{\prime} \neq k, \sigma_{A_{k}}^{\prime}$ should validate the choice of Eve (that is, play action 0 in $f_{k^{\prime}}$ - meaning that variable $x_{k^{\prime}}$ has value false). At state $f_{k}$ if $k$ is even, nothing can be done which could be profitable to player $A_{k}$ : state $u$ will be reached.

- A similar reasoning can be done for player $B_{k}$ : the only difference is at state $s_{k}$ when $k$ is odd, where player $B_{k}$ can only choose action 0 and go through $f_{k}$.

- In the part of the game after $b_{1}$, each player can deviate and choose to go to some state $c_{i}$; this choice will be made for checking the truth of clause $c_{i}$ under the valuation that has been fixed by the history so far.

Consider a deviation of some player that moves to $c_{i}$, and write $h$ for the corresponding history up to state $c_{i}$. The strategy of Eve after $h$ is to go to $d\left(\ell_{i, j}\right)$ where $\ell_{i, j}$ sets $c_{i}$ to true under valuation $\mathrm{v}_{h}$. If $d\left(\ell_{i, j}\right)=x_{k}$, then $(a)$ this means that $\mathrm{v}_{h}\left(x_{k}\right)=$ true, and (b) the next state is controlled by player $B_{k}$. Using the characterization of interesting deviating strategies above, it cannot be the case that player $B_{k}$ is the deviating player since from $t_{k}$ (which is visited by $h$ ), if only $B_{k}$ deviates, the game unavoidably goes to state $u$. Hence, for every strategy $\sigma_{B_{k}}^{\prime}$ for player $B_{k}$, history $h$ cannot be an outcome of $\sigma_{\text {Agt }}\left[B_{k} \mapsto \sigma_{B_{k}}^{\prime}\right]$. In particular, no deviation of player $B_{k}$ can lead to state $w$. Similarly, if $d\left(\ell_{i, j}\right)=\neg x_{m}$, the outcome ends up in $u$. In other words, each time a player other than Eve changes her strategy, the outcome ends up in $u$, yielding no improvement for the player.

Hence no player can improve her outcome by changing unilaterally her strategy, which shows that the strategy profile $\sigma_{\text {Agt }}$ is a Nash equilibrium where Eve wins. 
Now assume there is a Nash equilibrium $\sigma_{\text {Agt }}$ in which Eve wins. Let $v$ be a valuation such that for every $2 \leq k^{\prime} \leq p$ even,

$$
\left(\sigma_{\text {Eve }}\left(\mathrm{h}\left(v_{\mid\left\{x_{1}, \ldots, x_{k^{\prime}-1}\right\}}\right)\right)=1\right) \Leftrightarrow\left(v\left(x_{k^{\prime}}\right)=\text { true }\right)
$$

where $v_{\mid\left\{x_{1}, \ldots, x_{k^{\prime}-1}\right\}}$ is the valuation $v$ restricted to $\left\{x_{1}, \ldots, x_{k^{\prime}-1}\right\}$. We show the following two properties:

- if $v\left(x_{k}\right)=$ true then there is a strategy $\sigma_{A_{k}}^{\prime}$ for $A_{k}$ s.t. $\mathrm{h}(v) \in \operatorname{Out}^{\mathrm{f}}\left(\sigma_{\mathrm{Agt}}\left[A_{k} \mapsto \sigma_{A_{k}}^{\prime}\right]\right)$;

- if $v\left(x_{k}\right)=$ false then there is a strategy $\sigma_{B_{k}}^{\prime}$ for $B_{k}$ s.t. $\mathrm{h}(v) \in \operatorname{Out}^{\mathrm{f}}\left(\sigma_{\text {Agt }}\left[B_{k} \mapsto \sigma_{B_{k}}^{\prime}\right]\right)$.

We show the result by induction on the number of atomic propositions. For zero atomic propositions, the result obviously holds. Assume the result holds for atomic propositions $\left\{x_{1}, \ldots, x_{h-1}\right\}(h \leq p)$. Let $v$ be a valuation over $\left\{x_{1}, \ldots, x_{h}\right\}$, and $k$ such that $v\left(x_{k}\right)$ is true. Define $v^{\prime}$ as the restriction of $v$ to atomic propositions $\left\{x_{1}, \ldots, x_{h-1}\right\}$. By induction hypothesis, $\mathrm{h}\left(v^{\prime}\right)=s_{1} \cdot w_{1} \cdots w_{h-1} \cdot s_{h}$ is an outcome of some strategy $\sigma_{A_{k}}^{\prime}$ for player $A_{k}$.

- If $h$ is odd. Let $m_{\mathrm{Agt}}=\sigma_{\mathrm{Agt}}\left(\mathrm{h}\left(v^{\prime}\right)\right)$. We set $\sigma_{A_{k}}^{\prime}\left(\mathrm{h}\left(v^{\prime}\right)\right)$ to be 1 if $\bigotimes_{1 \leq k^{\prime} \leq p, k^{\prime} \leq k}\left(m_{A_{k^{\prime}}}=\right.$ $1) \otimes \bigotimes_{1 \leq k^{\prime} \leq p, k^{\prime} \neq k}\left(m_{B_{k^{\prime}}}=1\right)$ is different from $v\left(x_{h}\right)$, and to be 0 otherwise. Then we have that the next state is $t_{h}$ if, and only if, $v\left(x_{h}\right)$ is true.

- If $h$ is even, then the state after $s_{h}$ (actually after $\mathrm{h}\left(v^{\prime}\right)$ ) is $t_{h}$ if $v\left(x_{h}\right)$ is true, and $f_{h}$ otherwise, and this cannot be changed by player $A_{k}$. Then in $t_{h}$ and $f_{h}$ we set $\sigma_{A_{k}}^{\prime}\left(\mathrm{h}\left(v^{\prime}\right) t_{h}\right)$ (resp. $\left.\sigma_{A_{k}}^{\prime}\left(\mathrm{h}\left(v^{\prime}\right) f_{h}\right)\right)$ to be 1 . Note that since $v\left(x_{k}\right)$ is true we cannot reach $f_{k}$, hence setting the action of $A_{k}$ in those states to 1 always ensures that the next state is $s_{h+1}$.

This shows that $\mathrm{h}(v) \in \operatorname{Out}^{\mathrm{f}}\left(\sigma_{\mathrm{Agt}}\left[A_{k} \mapsto \sigma_{A_{k}}^{\prime}\right]\right)$ for some strategy $\sigma_{A_{k}}^{\prime}$.

The second property can be proven similarly for player $B_{k}$.

Let $v$ be a valuation satisfying condition (\#). We show that $\psi$ evaluates to true under that valuation. Let $c_{i}$ be a clause of $\psi$, and let $j=\sigma_{\text {Eve }}\left(\mathrm{h}(v) \cdot b_{1} \cdots b_{l} \cdot c_{l}\right)$. We show that $v\left(\ell_{i, j}\right)=$ true, which means that $c_{i}$ evaluates to true under $v$. This will show that formula $\psi$ is valid since condition (\#) defines sufficiently many witness valuations. Assume w.l.o.g. that $\ell_{i, j}=x_{k}$. Assume towards a contradiction that $v\left(x_{k}\right)=$ false. We have proven that there is a strategy $\sigma_{B_{k}}^{\prime}$ for player $B_{k}$ such that $\mathrm{h}(v) \cdot b_{1} \cdots b_{i} \cdot c_{i} \in \operatorname{Out}^{\mathrm{f}}\left(\sigma_{\mathrm{Agt}}\left[B_{k} \mapsto \sigma_{B_{k}}^{\prime}\right]\right)$. Now, the state $x_{k}$ is controlled by player $B_{k}$, so $B_{k}$ can enforce a visit to $w$ from $x_{k}$, so there is a strategy $\sigma_{B_{k}}^{\prime \prime}$ for player $B_{k}$ such that $\mathrm{h}(v) \cdot b_{1} \cdots b_{l} \cdot c_{l} \cdot x_{k} \in \operatorname{Out}^{\mathrm{f}}\left(\sigma_{\mathrm{Agt}}\left[B_{k} \mapsto \sigma_{B_{k}}^{\prime \prime}\right]\right)$. This contradicts the fact that $\sigma_{\text {Agt }}$ is a Nash equilibrium. We conclude that $v\left(x_{k}\right)=$ true, and we conclude that $\psi$ is valid (as explained above). 